\title{
Evidence of the Existence of a Large Amount of Cationic Antimicrobial Peptides in Propolis, with a Strong Physical/Electrostatic Mechanism of Action (Detergent-like), and their Possible Promising Effects in the Treatment of COVID-19 by means of Propolis Extract Inhalation
}

\author{
Eric A. Burger ${ }^{1}$ \\ ${ }^{1}$ Affiliation not available
}

June 16, 2020

\begin{abstract}
For more than a century, despite numerous documented therapeutic effects, extraction, administration and studies on propolis have all been focusing mostly on its phenolic compounds. However, some important components may have gone unnoticed and this perhaps has been preventing the exploitation of the full efficacy of the substance in the treatment of human infections/diseases. In the scientific literature there are already consistent clues that propolis main components, responsible for its medicinal properties, are, actually, antimicrobial peptides (AMPs). To investigate this possible existence of AMPs in propolis, experiments that can be easily reproduced were developed and, beyond not allowing the rejection of the hypothesis, may have revealed extraordinary evidence that the substance has a large amount of cationic AMPs, which produce strong effects. It was found that propolis, differently from the effects produced only by its phenolic compounds, has, given the significant hydrophobic residues of the water-soluble cationic peptides that abound in it, strong amphipathic/surfactant (detergent-like) character and its therapeutic mechanism of action is physical/structural, through electrostatic force. In order to produce effects, the cationic peptides in propolis bind, by attraction, to anionic moieties of the organism/agent with which the substance interacts, and generate a cascade of phenomena. Thus, the results of the experiments developed suggest that there is a potential that should be extensively explored by science; i.e., propolis and its cationic AMPs possibly have strong antimicrobial and disease fighting properties and are designed to not be resisted by any pathogen, mainly if applied directly to the infection/disease site in the necessary amount. The experiments may also have revealed, as studies have been demonstrating that propolis is non-toxic and safe to be used by humans even in large doses, a possible powerful therapeutic agent that is ready to be utilized now against SARS-CoV-2. Propolis existing extracts may be easily tested for effectiveness and their use for prophylaxis or treatment of COVID-19 may be quite simple given that the substance, as demonstrated, seems to be basically a more complex and selective/non-toxic "detergent/soap" that humans have been safely using internally. Inhalation of extracts may be highly effective and the best existing way for propolis parenteral administration, enhancing the absorption and systemic effects; it may also allow direct action against SARS-CoV-2 in the respiratory tract - possible prompt binding of the positively charged AMPs from propolis to all negatively charged parts of the viruses/host's cells and their products/substances, potentially annihilating the virus and producing immunomodulatory effects. In conclusion, propolis may be able to bring together, in the same substance, the expected effects of various therapeutic agents, and, as a result, it is possibly a viable and promising treatment alternative for COVID-19, especially in comparison with the traditional drugs being tested at the moment. Through the present study it was demonstrated as well that propolis has sufficient preclinical proof and safety to be tested in humans; therefore, considering the current pandemic, it is suggested that further studies on the substance should start urgently, primarily clinical trials on its possible efficacy in the treatment of COVID-19.
\end{abstract}


Eric A. Burger

burger. eab@gmail. com

*Preprint version, not peer-reviewed (C)

\section{Introduction}

Given the current COVID-19 planetary pandemic, which has been causing thousands of deaths, the present evidence-based essay, with the aim of trying to save lives, was developed in a very short time. Despite respecting scientific research criteria and the contents that speak for themselves, as a work that could have been divided into separate articles, it does not intend to be solely a succinct report of an experiment. It is different; it is a more robust study, in which, in order to establish a solid background, construct and demonstrate a plausible hypothesis, expand the discussions and allow a better comprehension of the theme, the divisions and subdivisions were done in an instructive form and the subjects investigated/discussed extensively. It is broader than traditional articles; a hybrid of the description of an experiment and some "reviews" in several fields to help in the contextualization and discussions; with also some anecdotal evidence in the end to encourage further testing. All this is due to the fact that, besides the complexity of the theme itself and the impossibility of carrying out advanced laboratory and clinical trials in an independent work, it is believed that the outcome of the present research has the potential to save lives inside this devastating pandemic and hence must be rapidly released in a complete manner. The study, beyond the evidence, is also intended to serve as a possible comprehensive treatment guide, ready to be used. COVID-19 is not a simple issue, so, the most important information was gathered and condensed to facilitate the analysis.

Thereby, the primary purpose is simply to convey knowledge backed by science, readily sharing the findings with the scientific community. What is believed to be an extraordinary and promising treatment hypothesis for COVID-19 and a possible solution for this devastating disease cannot waste any time. Thus, observing formal and methodological prerequisites, the study, independently of specific predefined formats, must be released quickly in order to allow other researchers and health professionals to freely review it and maybe put the findings to clinical test immediately. "Desperate times call for desperate measures" and it is extremely necessary to think "out of the box", with a holistic and multidisciplinary scientific approach of this complex question. Considering the limitation of resources of an independent research, the study was developed mostly through "reversed engineering" - from a possible successful treatment to thorough investigation and experiments to find the reasons; the specific implications of the findings in the treatment of SARS-CoV-2, and comparisons with existing drugs that are being tested against the virus at the moment.

In the current pandemic, it is a notorious fact that the World Health Organization (WHO) and researchers all over the world are desperate to find an effective mean to fight COVID-19. The main course of action at the moment is drug repurposing of chloroquine or antivirals, both either isolated or in combination with antibiotic or anti-inflammatory. So, the specific objective of this study is to present and explore propolis as a viable complete treatment for COVID-19. It will be demonstrated, with several clues in the scientific literature and with experimental evidence, a possible therapeutic mechanism of action never explored before in propolis. In the scientific community, propolis is mostly regarded as just a folk medicine supplement, thus a convincing study was necessary to try to demonstrate its powerful medicinal properties. Beyond the existing clues in the studies developed until now, extraordinary evidence may have been found through the experiments developed in the present research, demonstrating that propolis possibly has a strong and never explored mechanism of action to fight diseases. Also, propolis basic pharmacology was explored - suggesting a potential way to test the efficacy and administrate it. Through the study of SARS-Cov-2 specificities and in a comparison with the traditional drugs currently in test, it was possible to suggest that propolis administration, especially by means of inhalation, may be a viable and an effective treatment against COVID-19. In the end, as said, some anecdotal evidence of propolis possible effects on the treatment of respiratory tract infections (possible COVID-19) was brought.

As a final observation, it is worth mentioning that, in order to enhance the comprehension and allow quick reading, the most important considerations were highlighted throughout the study. In addition, for the sake 
of terminological precision, it is also worth to clarify that, despite quoting them several times as simply "bees", most of the references utilized in this study use the terms "honey bees" or "honeybees" - usually referring to the most common bees from the genus Apis, which are, in a stricter sense, the only true "honey bees", mainly Apis melifera, the domestic honeybee. In the present work, the terms "bee" or "bees" were chosen to simplify and as well as a general reference for all bees that produce propolis - not only honeybees, but others such as, e.g., stingless bees. This is due to the fact that the focus of the present study is on propolis and, as will be seen, all propolis types, regardless of the kind of bees that produce them, may be capable of producing therapeutic effects.

\section{Possible Mechanism of Action of Propolis on Microbes and Antimicrobial Peptides (AMPs)}

\subsection{Propolis as Bees' Social Immunity Tool and Studies on the Effects of Propolis on Human Diseases}

The first scientific work with propolis was published in 1908 . Propolis has been known for centuries as a medicine and it is used nowadays generally as a dietary supplement. It is also known as "Russian penicillin " and is the substance that bees use to protect their hive against pathogens of any species, including virus, bacteria, fungi or protozoan . Most studies about propolis are concentrated on its antimicrobial effects on human health and its chemical compounds, with some in the field of social immunity, in the insect biology area, trying to explain the importance of propolis in regards to bee health. These studies demonstrate that propolis in the nest allows bees to invest less in their immune systems, preserving their immune system gene expression and energy in order to raise their colony productivity and survival rates .

It was not certain if resin collection for propolis production may be constitutive (i.e., collected regardless of physiological demand or pathogen level) or inducible (i.e., a conditional response to infection; a form of self-medication) . However, the same authors, in another study lasting three years found that it is inducible , i.e.,bees raise resin collection and propolis production in response to colony infection, as a particularly unique example of self-medication operating at the colony level (social immunity ). They do not ingest propolis, but use it within the hive - e.g., adult bees exposed to fungal spores . Bees alsoneed the propolis envelope to protect the whole colony . So, as bees do not naturally consume propolis , the mode of action of a therapeutic effect of propolis on colony pathogens is probably via volatile compounds or direct contact either on the hive walls, a barrier at the nest entrance, or along the rims of comb cells. It is believed that these extraordinary findings show the broad ability of bees to respond to infections just raising bees that forage resins and augmenting propolis quantities inside the colony - possibly not quality or composition. It seems to be a powerful social immunity instrument in the colony, as if it were a crucial bodily system inside this living organism that is the hive and its constituents. This also leads to the conclusion that the use of propolis by humans is like "borrowing from bees their most important immune system" for a while.

There is a significant amount of studies about the effects of propolis on diseases and the majority are just in vitro cell-based assays and assays in rodents, with only a few in humans . The focus of the existing studies, as will be discussed, is basically on propolis phenolic compounds, which may have hindered the exploitation of other powerful medicinal components. In addition, as it is believed and will be seen throughout this work, most pharmacological studies tend to concentrate only in one compound and its consequent chemical reactions/effects, not considering the possible physical variables involved in the processes. There are few clinical trials to prove the effects of propolis possibly because of the lack of interest of the pharmaceutical industry in the substance - maybe given that it is difficult to patent naturally occurring compounds. It is also supposed that natural substances do not attract health professionals (heavily trained only in allopathic therapies) mainly because the pharmaceutical industry obviously does not finance and sell for them the idea of researching and using natural substances in the treatment of patients like it has been extensively doing for years with traditional drugs. Thus, the advance in research about propolis has always depended on some interested academic researchers and small producers and industry, with very limited financing resources.

In spite of this situation, propolis has been showing antimicrobial; wound healing and cardio-protective 
effects, which is related to its demonstrated antioxidant and anti-inflammatory activity . Antimicrobial properties include, for example, specifically relating to antiviral activity : anti-influenza A effects, corresponding to the effects of oseltamivir ; anti-HSV 1 and $\mathbf{2}$, demonstrating better effects in comparison to acyclovir ; effects on human rhinovirus HRV-2, HRV-3, and HRV-4, showing more pronounced antiviral activity than ribavirin ; anti HIV ; and anti Vaccinia, Hepatitis, Polio, measles, rubella and Picornavirus. Moreover, propolis has demonstrated activity against Gram-positive and Gramnegative, as well as aerobic and anaerobic bacteria . Anti-plasmodial effects against malaria were demonstrated as well, with no significant difference in survivorship of rodents infected with malaria parasite, Plasmodium berghei, treated using propolis orchloroquine . Propolis has also immunomodulatory effects on the infected organism as will be shown below. It is believed that this high efficacy in a wide range of microbes and the immunomodulatory effects may be attributed to the special nature of propolis that is going to be demonstrated throughout the present work.

\subsection{Effects of Propolis on Host's Cells and Immune System}

Propolis, in line with what is going to be seen about its possible nature, has been showing, in vitro and in vivo, specifically in regards to viral infections, promising effects on the infected host cells and on the modulation of the immune response. Most trials use rodents to test the effects of propolis in vivo and the majority have very positive effects. Despite mostly related only to the phenolic compounds, some of the documented effects that propolis may have against viruses are summarized in the chart below - mainly regarding respiratory tract infections, which is line with the aim of the present study.

\section{Propolis Caffeic acid phenethyl Ester (CAPE) blocks the NF-kappaB activation process}

Propolis has immunomodulatory effects on macrophages

Brazilian Green Propolis 3,4-diCQA compound increases TRAIL (TNF-related apoptosis-inducing ligand) Brazilian Green Propolis Artepillin C (3,5-diprenyl-4-hydroxycinnamic acid) increases the ratio of CD4/CD

\subsection{Other Effects of Propolis that May Help during an Infection}

Propolis may also help the infected organism through a series of other benefits. The most important property is the general anti-inflammatory activity of propolis, which is not chemically full understoodand generally attributed to its phenolic compounds, through five main demonstrated mechanisms: (a) the inhibition of cyclooxygenase (COX) and consequent inhibition of prostaglandin biosynthesis; (b) free radical scavenging - powerful antioxidant effects of polyphenols and flavonoids; (c) inhibition of nitric oxide synthesis; (d) reduction in the concentration of inflammatory cytokines; and (e) immunosuppressive activity

The anti-inflammatory effects are quite interesting, with a randomized controlled study, one in a few study in humans, demonstrating that Brazilian Green Propolis improves antioxidant function in patients with type 2 diabetes mellitus, modulating the inflammatory response to chronic inflammation - which may be an evidence-based advantage for the use of propolis in the treatment of this kind of patients, because they are more susceptible to acute inflammation complications. Interestingly, a randomized doubleblind clinical trial also in humans found that the intake of propolis resulted in improvement of glycemic and some serum lipid levels in patients with type 2 diabetes mellitus .

Regarding respiratory tract inflammation, which is a very important matter for the objective of the present work, studies brought strong evidence of propolis effectiveness against it. A study in mice demonstrated propolis potential antioxidant and anti-inflammatory role even in acute lung inflammation caused by chronic smoking, observing also an increase in alveolar macrophages and neutrophils in the chronic smoking group compared with the Control . Also, in a study that used propolis fromScaptotrigona aff. postica stingless bee, the substance showed similar results in comparison todexamethasone in the treatment of murine asthma due to an inhibition of both the inflammatory cells migration to the alveolar space and the systemic progression of the allergic inflammation. When tested in 
humans - children -, propolis, combined to echinacea and vitamin C, showed apreventive effect on the incidence of respiratory tract infections. Also in children, another study in Italy showed that the use of propolis supplement for $\mathbf{7 2}$ hourslessened the severity of acute otitis media and viral pharyngitis, reduced the use of antipyretics and anti-inflammatory drugs, and decreased the rate of evolution to tracheitis, bronchitis, and rhinosinusitis, concluding that propolis could be used as a safe add-on therapy in case of acute otitis media and/or viral pharyngitis. This possibly demonstrates, again, propolis promising effects not only in rodents, but also in the human respiratory tract .

Another singular property described in clinical investigation in mice and humans is that propolis and its constituents are generally well tolerated and non-toxic, unless administered in very large quantities. It was found only one anecdotal evidence of kidney failure and some indications that propolis may decrease kidney perfusion; however, it was a long term treatment, the patient studied had a serious comorbidity and the kidney function improved after propolis withdrawal .In comparison to other antimicrobial and anti-inflammatory drugs, the lack of side-effects of propolis is notorious . Someallergic reactions may happen, but they can be easily avoided, mainly in children and adolescents, with a simple patch test before prescribing. Tests in children have been demonstrating propolis safety, even in small children. Actually, as already commented, tests in murine asthma showed an inhibition of both the inflammatory cells migration to the alveolar space and the systemic progression of the allergic inflammation, demonstrating thatpropolis may also act as anti-allergic agent . It is supposed that all these immunomodulatory properties and the general absence of side effects may help the infected organism to preserve and restore the homeostasis, not putting more stress on the overburdened immune system, but, on the contrary, enhancing host's general ability to fight the inflammation caused by microbial infection.

Lastly, propolis has some demonstrated probiotic nature andmay have some benefic effect on intestinal barrier function, but overall, the degree that propolis supports the health of the intestinal microflora has been poorly investigated with longer term studies needed on the effect of dietary polyphenols on gut microbiota, an area worthy of further research. No adverse effects of propolis on gut health were documented in the studies quoted so far - propolis may not strongly harm the gut, especially compared to the notorious harmful effects of antibiotics. However, as will be also discussed, it is suggested that further studies are necessary on the effects of propolis in gut health and, considering that the substance is a potent antimicrobial, despite polyphenol-rich, propolis may not be recommended for long term and/or heavy dosage treatments via oral administration, as it may disrupt the natural gut microbiome and balance.

\subsection{Direct Effects of Propolis on Pathogens}

Propolis has demonstrated direct effects against viruses, which is no surprise as science has already proven that the substance is utilized to protect the hive against microbes. There are promising documented direct effects of propolis against various types of viruses. Propolis is effective against various RNA viruses and also a retrovirus such as HIV and is able to lower viral number of copies and replication velocity in vitro, demonstrating similar or superior effects compared to antiviral drugs such as oseltamivir or acyclovir. Themechanism of action and which are the specific components that bring these effects, given the hundreds of components in the substance,despite some hypotheses, are not exactly understood by the studies cited, with most speculations relating only to propolis phenolic compounds.

For example, a recent study in Korea, with cell cultures, aiming to demonstrate the possible mechanism of action of Brazilian propolisphenolic compounds kaempferol (KF) and $p$-coumaric acid ( $p$-CA) and other 8 compounds, showed efficacy for most of them in human rhinovirus HRV-2, HRV-3, and HRV-4. Some compounds had more pronounced antiviral activity than ribavirin, with KF and $p$ -CA remarkably reducing the RNA replication levels of HRV-3 in the cell cultures and also inhibiting HRV-3 infection when added during the early stages $(0-4 \mathrm{~h})$ after virus inoculation . Thus, the study suggested that $\mathrm{KF}$ and $p$-CAmay block or reduce the entrance of the viruses into the cells to protect the cells 
from the virus destruction and abate virus replication, which may play a crucial role in interfering with expressions of intercellular adhesion molecule- 1 and interleukin- 6 . As seen, even the isolated phenolic compounds in propolis (which, as will be exposed, are not the objective of this study and may not be necessary) are able to produce promising effects .

Although conducted in bacteria (antibiotic-resistantStaphylococcus aureus and antibiotic-sensitive Escherichia coli ) rather than viruses, a brilliant American study with Russian propolis ethanolic extract (RPEE) found that, ipsis literis :

The activity of Russian propolis ethanol extracts (RPEE) towards both Gram-positive and Gram-negative bacteria has already been evaluated (Parolia et al. 2010). Silici and Kutluca 2005; report that propolis is active towards Gram-positive bacteria, but is only active towards Gram-negative bacteria at high treatment amounts. This may be due to the differences in constituent arrangement and composition between Gram-positive and Gram-negative bacteria cell wall structures. The viability of Staph. aureus planktonic bacteria decreased with respect to increasing treatments of RPEE (Fig. 1a). RPEE showed different antibacterial activity towards E. coli when compared to Staph. aureus planktonic bacteria. As expected, the activity of RPEE decreased when treating E. coli, due to the differences in the chemical and physical components of Gram-negative bacteria (Fig. 1a). Up until $3 \% \mathrm{w} / \mathrm{v}$ of propolis, significant numbers of E. coli bacteria were still viable. On the other hand, the numbers of viable Staph. aureus planktonic bacteria were significantly reduced when treated with the same $3 \% \mathrm{w} / v$ of propolis (Fig. 1a). Beyond $\mathbf{5 \%} \boldsymbol{w} / \boldsymbol{v}$ of RPEE, the bacteria become overwhelmed and complete planktonic bacterial death is observed for both strains. Based from the results generated in this study, it is believed that differences in the extra lipolysaccharide layer of Gram-negative bacteria, act as an extra protective coating around the cell membrane, reducing RPEE exposure to the cell membrane. As a result, more of the antibacterial compounds found in RPEE are needed to interact specifically with the cell membrane and achieve the same inhibitory effect. (emphasis mine)And continues :Most studies believe the mechanism of action of propolis is functional rather than structural. In these studies, the antibacterial activity is attributed to a synergism between the compounds in the resin (Koo et al. 2000; Park et al. 2000). In previously published work, propolis is said to inhibit bacterial adherence, division, inhibition of water insoluble glucan formation and protein synthesis. Although, these chemical mechanisms of action may be plausible, they do not consider the excellent ability of bacteria to evolve and gradually adapt and acquire resistance to the chemicals found in propolis. An example of bacteria's uncanny ability to adapt and thrive would be their resistance towards antibiotics. The results uncovered in this work demonstrate that RPEE has an antibacterial mechanism similar to lytic peptides, which induce bacterial death through cell lysis. We found in our previous study that Russian propolis ethanol extracts contain bothantibacterial (bacterial killing) compounds and anti-biofilm (biofilm removal) compounds (Bryan et al. 2015). It is believed that as sufficient amounts of RPEE come into contact with biofilms, the chemical and structural integrity of the biofilm become compromised by the anti-biofilm compounds in the RPEE. As damage to the extracellular polymeric matrix becomes more severe, removal of the coating from the surface of the biofilm occurs, resulting in reduction in thickness of the biofilms. As a result, the polymeric coating is no longer able to provide protection to the bacteria. The inability of the biofilm to protect the infectious micro-organisms allows access of the antibacterial compounds to the bacteria (Bryan et al. 2015). Once the bacteria come in contact with the RPEE, the antibacterial compoundsbind specifically to the cell membrane of bacteria,resulting in catastrophic cell lysis and eventually cell death. The mechanism of action of the Russian propolis ethanol extracts towards both Gram-positive and Gram-negative bacteria seems much different than what has been previously proposed. Other groups believe the mechanism to be very specific in their targets. However, the results from this study show a totally different means of inactivation. The physical nature of the propolis extracts renders this antibacterial a much more attractive substitute for traditional antibiotics. In fact, RPEE may be compared to Cationic Antimicrobial Peptides and offers several attractive advantages including their activity at very low amounts and the unlikelihood of bacterial resistance due to their physical nature. A conceivable downside involving 
the physical nature of this antibacterial is the possibility of toxicity towards tissue cell and will need to be evaluated. Also, determining the mechanism of action of different propolis samples from different origins, along with characterizing and establishing between the antibacterial and anti-biofilm compounds involved in the mechanism of action will also need to be determined. (emphasis mine)

This extraordinary physical mechanism of action is being slowly indirectly proven by studies that demonstrate the synergism between propolis ethanolic extract and antimicrobial drugs - especially those agents that interfere on bacterial protein synthesis such as choramphenicol, gentamicin, netilmicin, tetracycline and vancomycin . In addition, the absence of antagonism between propolis ethanolic extract and antimicrobial drugs was found, with the studyhypothesizing the potential medical use of propolis in combination with certain antimicrobial drugs in staphylococci diseases, since bacteria may be resistant to several antimicrobial drugs. Other demonstration of propolis synergistic effects is a study showing significant results in combinations of cefixime and propolis when compared to infected controls, concluding that propolis acted synergistically with cefixime, enhanced the efficacy of the antibiotic and reduced its effective dose in combined therapy .

As the American study above also stated, in $\mathbf{2 0 1 6}$, there are only a small number of studies focusing on the inhibitory effect of propolis against bacteria within mature biofilms and an evenfewer number of studies interested in the mechanism of action, therefore, the inactivation of bacterial biofilms by means of propolis is still relatively novel, with many unanswered questions . The results discussed in the study have started to dissect the mechanism of action of propolis extracts and provide evidence for the successful treatment of both Staphylococcus aureus and Escherichia coli biofilm-contaminated surfaces .

\subsubsection{Initial Discussion of Toxicity, Mechanism of Action and Compounds, and Dose Dependence of Propolis}

Notwithstanding the quoted American study stating in the end, as a conceivable downside of propolis use, the possibility of toxicity towards tissue cell, it will be showed below that most studies in vitro or in vivo have not shown any toxicity directly on cellsor in the infected organism, but the contrary - In this way, another recent study, with two of the researchers that developed the American study in discussion, suggested that the structural mechanism of action of Russian propolis ethanol extracts stem predominantly from the organic compounds, and when metals were removed from it there was a reduction of toxicity to mammalian cells, suggesting a potential for medical and biomedical applications. It is believed that these metals may be present given the use of pesticides, which can be solved by using organic propolis. Besides this, the physical mechanism of action of propolis may be selective, given, as will be detailed below, that it is possibly composed mostly by AMPs (antimicrobial peptides) - which the own American study pointed. This selective electrostatic mechanism - which will be further extensively discussed - preserves human cells from damage, because of the lower cytotoxicity due to the fact that host cell membranes possess a higher percentage of cholesterol . Beyond this, also regarding electrostatic reactions, the cationic property of AMPs mainly contributes to cell selectivity because the surface of bacterial membranes - and sugar chains of virus glycoproteins - aremore negatively charged than that of mammalian cells .

In the end, the American study stressed the necessity of determining the mechanism of action of different propolis samples from different origins and the compounds involved in this mechanism. This is believed to be impracticable given the countless compounds existent in nature that, as studies have been demonstrating, varies a lot between regions where the hives are located. The theme will be examined below, with a different view, suggesting the possibility that all propolis types from anywhere have the potential to be effective against microbes, as a study, with a focus on the phenolic compounds, already stated .

It is worth to highlight, besides the effects through the infected organism, the main direct mechanism of propolis action on microbes, as mentioned in the American study, may be physical, structural - Nevertheless, different from what have been hypothesised, it is believed that this mechanism, as other studies on phenolic compounds have been pointing, is due to the synergy between all the compounds 
in propolis, and not due to one or two compounds isolated, and, as will be seen, may be also very specific on the targets. This possibly happens because, as the own American study demonstrates, propolis has a variety of compounds with different lines of action, such as antibacterial (bacterial killing) compounds and anti-biofilm (biofilm removal) compounds, and this reasonably demonstrates that one mechanism needs the other to be effective. That is why it is suggested that the tentative to isolate one or only a few compounds of propolis may break this intricate mechanism and may make impossible to keep satisfactory antimicrobial effects. As will be discussed and showed below, the compounds of propolis vary a lot according to the location that the substance is extracted - despitehaving common compounds present in many samples from different places -, and also depend on correct extraction methods.

In addition, despite the fact that bees, from place to place, alter the feedstock used by their "high-tech laboratories" to produce propolis, it is believed that the immunity-like result achieved, due to the synergy of compounds utilized - which possibly generates aunique electrostatic/physical profile, as will be detailed -, is quite the same for all propolis types. The capability of selecting ready components and ingredients from plants, also synthesizing own components with them to achieve the necessary result for the colony survival, engineering a complex and intricate ancient mechanism of defense - which possibly evolved through transient de novo synthesis (memory formation) of a wide range of antimicrobial peptides (AMPs) -, may have contributed to the low, if any, resistance from pathogens to propolis. This genetically programmed mechanism may have allowed the resilience and adaptation of bees in an immense variety of environments, responding to infections and, depending on the aggressor, just raising the number of resin foragers - and, maybe sometimes the expression of immunity-related genes, increasing certain AMPs to raise individual immunity. As can be seen, it is a matter of dose dependence as well, with bees just augmenting the amount of propolis produced inside the colony (a living organism) to inactivate and clear the invader.

As the American study demonstrated, the main mechanism of action of propolis is achieved through structural/physical damagedirectly inflicted on invaders, and possibly just in adose-dependent manner - it is worth to stress that avery low concentration was used in the study, with only $5 \% \mathrm{w} / \mathbf{v}$ in the RPEE. Therefore, it is also believed that, in line with the entomology studies already commented above, which related propolis to social immunity, propolis is a genetically pre-conceived complex final "immune system" of the living organism that the colony is. Thus, that is why propolis cannot be fractioned, and depends on the synergy of its compounds - synergy already proposed in various studies. It is a "system" that has to and will be reproduced and achieve its final outcome (protection of the hive against all pathogens) regardless of the compounds utilized for its production. Perhaps this is why thespectacular mechanism of action of propolis differs from any known drug ever produced. This process is supposed to be the same in any other living organism, as a genetically preprogrammed feature. Humans, which are much younger than bees in evolutionary terms, for example, before civilization, were known, in a simple view, for the capability of changing food sources according to the place they lived and still obtain the feedstock to achieve the same final outcome that is "synthesizing" or keeping a healthy immune system - this also possibly allowed adaptation in an enormous variety of environments.

It is worth highlighting that, given this suggested special nature,propolis dose dependence to achieve its treatment efficacy and beneficial effects has been explored, besides the American study under scrutiny, in studies about several therapeutic properties of the substance, such as leukocyte and protein profiles of mice and wound healing ; in vitro cancer fighting, antimicrobial and immunomodulatory properties, and intestinal tissue of rats and immunity . Even atime dependence of a treatment was also observed, which will all be detailed below. This believed necessity of propolis in the colony and/or raising dosage according to the pathogen, to avoid its resistance by physical (electrostatic action, as will be seen)and not any biochemical transformation, may be why abacterial disease called American Foulbrood has infected between 3 and 15 percent of all honeybee colonies worldwide. The infection possibly derived from human interventions that resulted in a lack of a propolis envelope inside the hive, diminishing bee defenses. Given this lack of defense, as expected, the best remedy for American Foulbrood seems to be propolis - which also may be true for other bee parasites such as Ectoparasitic Mite Varroa destructor and its associated viruses in which propolis showed to be really important for honeybee health . Another 
study also demonstrated the hypothesis thatresin collection and propolis use in the hive represent an example of self-medication behavior in social insects. In European-bees, the domestication of them has resulted in a reduction of resin collection and less propolis production, whichpossibly is another evidence of thedose-dependent mechanism that varies according to environmental challenge and pathogens presence.

In this way, as seen, all evidence brought, by association, point toward the importance of dosage and direct action of propolis on the invader, which will be seen below. Furthermore, these findings - especially about American Foulbrood and the findings brought by the American study quoted above - preliminarily allow to conclude that propolis is designed to not be resisted by any microbesand any resistance is caused by unexpected factors like human interventions - in this case, impossibility to direct achieve the invader because of the absence of an envelope and the absence of enough substance to clear the infection . So, as suggested and partially demonstrated, and will be further detailed, to summarize the discussion and arguments, propolis has possibly low toxicity and a special mechanism of action (which depends on the synergy of its compounds and is dose-dependent) that is designed to not be resisted by microbes .

\subsection{Antimicrobial peptides (AMPs)}

In order to allow the development of the present work it is necessary to correctly define antimicrobial peptides (AMPs) - also remembering that the American study mentioned above compared propolis extract to cationic antimicrobial peptides. It is known that plants lack adaptive immunity (i.e., B cell and T cell mediated immunity) and hence AMPs (antimicrobial peptides) play a fundamental role in their protection against infection. The same is true for bees and all invertebrates that do not have an adaptive immune system and need these peptides for survival. AMPs are also known as host defense peptides, are short and generally positively charged peptides found in a wide variety of life forms from microorganisms to humans - these special peptides will be called AMPs; term considered more comprehensive and appropriate for the objective of the present work. During the past two decades, several AMPs have been isolated from a wide variety of animals, both vertebrates and invertebrates, and plants, as well as from bacteria and fungi . Most of these peptides are obtained from different sources like macrophages, neutrophils, epithelial cells, haemocytes, fat body, reproductive tract, etc .In humans, over $\mathbf{1 0 0}$ such peptides have been identified from a variety of tissues and epithelial surfaces, including skin, eyes, ears, mouths, gut, immune, nervous and urinary systems, and are key components of innate immunity, playing a critical role in warding off invading microbial pathogens, with other biological functions such as apoptosis, wound healing, and immune modulation . This arsenal of human defensive proteins are not only responsible for health but also inspires the development of a new generation of personalized medicine to combat drug-resistant superbugs, fungi, viruses, parasites, or cancer . It also seems that AMPs could be considered to possess both pro-inflammatory and anti-inflammatory properties , suggesting that they are key players in the inflammatory microenvironment, and, as will be seen, theyact on free-radicals through a more complex mechanism, not only through free radicals scavenging

AMPs have been receiving a lot of attention from science in the last years given their broad spectrum of antimicrobial activity, including anti-bacteria, anti-fungi, anti-viruses, cancer fighting properties, and important role in immunomodulatory and inflammation responses . Nowadays, as it is believed and will be shown - trying to do what bees have already been perfectly doing for millions of years, with a high-tech mechanism of action, mostly through physical attack against invaders, given propolis unique properties -, science is focusing in trying to extract AMPs from many sources or in a way to mimic AMPs actions and apply it to treat various illnesses. As an example, a new technology in development uses nano-sized particles of magnetic liquid metal to shred bacteria and bacterial biofilm in order to avoid antibiotic resistance that every year kills thousands of people .

In a brief explanation, to help clearing the arguments for propolis direct efficacy that will be shown below, it is worth mentioning that, although AMPs differ in sequence and structure, they share common features, which 
are their overall cationic charge, asignificant fraction of hydrophobic residues and anensuing amphipathic character. Regarding host's innate defense against bacteria, despite other actions on host's cells that will be explored as well, AMPs act firstly by the property ofattraction, which is presumed to occur when the initial interactions between the cationic peptides occur via electrostatic interactions with negatively charged moieties in the bacterial membrane, with pronounced interactions of the peptides with the phospholipid membranes . In contrast, as said,AMPs display lower cytotoxicity to host's cells due to the fact that their membranes possess a higher percentage of cholesterol. As also said, the cationic property of AMPs mainly contributes to cell selectivity, because the surface of bacterial membranes - and sugar chains of virus glycoproteins - aremore negatively charged than that of mammalian cells .

Following the mechanism of action, the second step of AMPs isattachment, as the peptides must now traverse the exterior capsular polysaccharides to reach the inner lipid layer .Lastly, during the peptidemembrane interaction two physically distinct states occur : the membrane-stretching and, after raising the peptide/lipid ratio, pores begin to form in the critically thin membrane, and the peptides orient themselves perpendicularly and insert into the bilayer . After insertion, several models have been developed that explain how AMPs kill organisms through membrane permeation . It is not convenient to detail all the models in the present study, just stressing that, one of the models, similarly to the others, states that, as theconcentration increases it is thought that the peptides intercalate into the membrane in a detergent like manner causing the bilayers to continuously bend so the water core is lined by both the inserted peptides and the lipid head groups and, once a critical threshold concentration has been reached, the membrane disintegrates and forms micelles .

The amphipathic character of AMPs and their interactions with membranes resemble the properties of detergent molecules and analogies between membrane-active peptide and detergents have been studied, with several models suggesting to explain the pore-forming, membrane-lytic and antibiotic activities of these peptides. Many species of life containcationic antimicrobial peptides as components of their immune systems, thus, the antimicrobial activity of these peptides has been studied extensively, and many peptides have a broad spectrum of activity not only against gram-negative and gram-positive bacteria but also against antibiotic-resistant bacteria, fungi, viruses, and parasites . Such cationic antimicrobial peptidescan also act in synergy with host molecules, such as other cationic peptides and proteins, lysozyme, and also conventional antibiotics, to kill microbes and it has been found thatcertain peptides are produced in large quantities at sites of infection/inflammation, and their expression can be induced by bacterial products such as endotoxic lipopolysaccharide (LPS) and pro-inflammatory cytokines, such as tumor necrosis factor-alpha (TNF-alpha) . It is worth stressing the known fact that pro-inflammatory cytokines also raises in response to viral infections, which, consequently, as stated, increases the expression of host's AMPs in the site of infection, allowing them to possibly interact with "implanted" external AMPs. These peptides often have a high affinity for bacterial products, such as LPS, allowing them to modulate the host response and reduce the inflammatory response in sepsis and, more recently, they have been found to interact directly with host cells to modulate the inflammatory process and innate defenses .

These components are so powerful that certain AMPs show their inhibitory effects on Gram-negative and Gram-positive bacteria via not only destroying bacterial cell membranes and subsequently leading to the cell death, but also entering the cytoplasm and binding to DNA, disturbing bacterial physiological activity. That is, AMPs act in a more powerful way when compared with detergents/emulsifiers, not only trapping the invader but also "destabilizing" it . There are other mechanisms as well, as it will be shown in the section below regarding antiviral mechanisms of AMPs. As shown, mainly referring to bacteria, the main mechanism of action of AMPs is to interact with the bacterial membrane - and several models have been developed to explain how AMPs kill organisms through membrane permeation -basically killing it by physical/mechanical cell lysis ,mechanism which , as will be discussed below, may have some differences for viruses . 


\subsection{Effects of Antimicrobial Peptides (AMPs) on Viruses}

As the American study mentioned above is about the effects of propolis on bacteria and most considerations in the previous section were about bacteria, it is valid to explore as well, according to the scope of this study, the mechanisms of the AMPs specifically on viruses. Thus, to spare time, the effects will be summarized quoting part of a complete review about AMPs:

Antiviral AMPs neutralize viruses by integrating in either the viral envelope or the host cell membrane. Previous studies have shown that both enveloped $R N A$ and DNA viruses can be targeted by antiviral AMPs. AMPs can integrate into viral envelopes and causemembrane instability, rendering the viruses unable to infect host cells. AMPs can also reduce the binding of viruses to host cells. For example, defensins bind to the viral glycoproteins making herpes simplex viruses (HSV) unable to bind to the surface

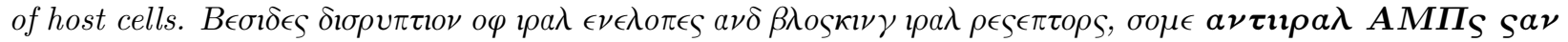

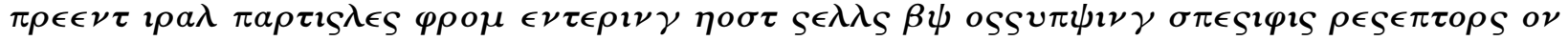

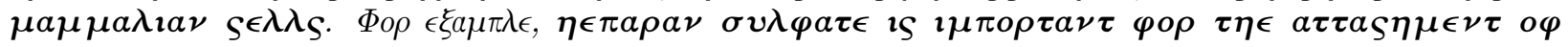

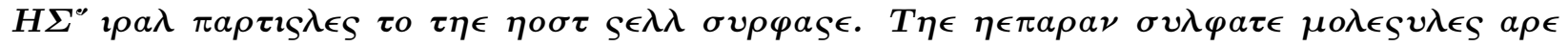

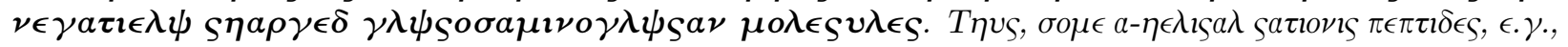

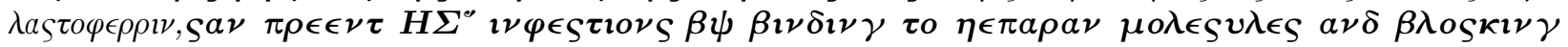

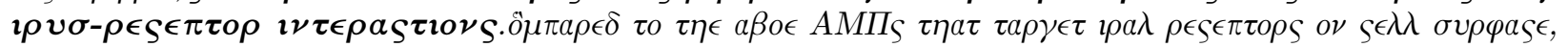

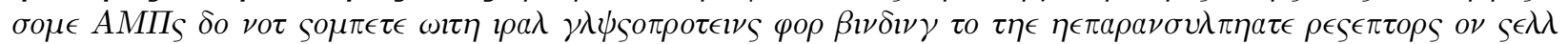

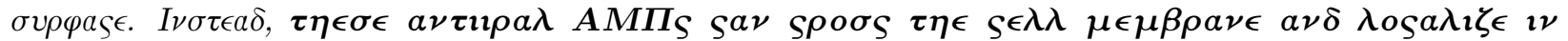

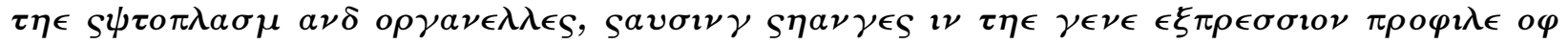

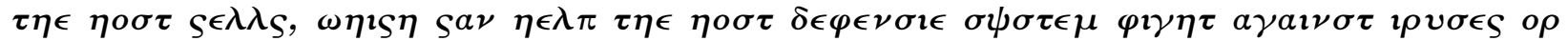

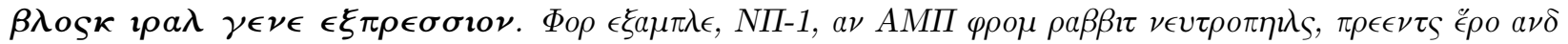

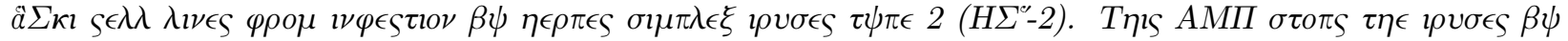

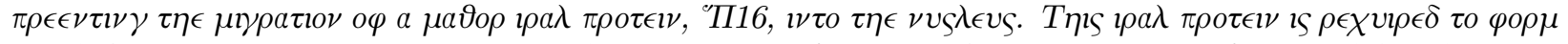

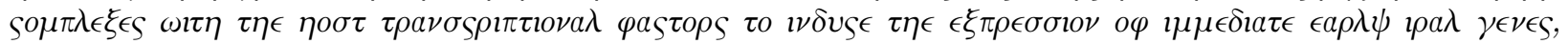

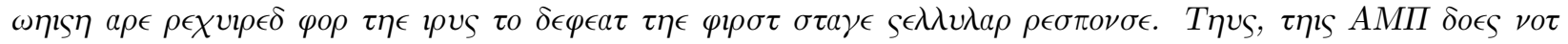

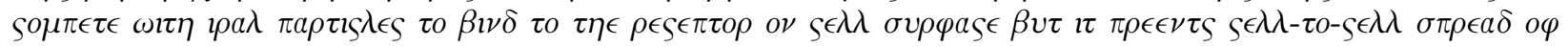
$\iota \rho a \lambda \pi a \rho \tau \imath \zeta \lambda \epsilon \varsigma$. ( $\epsilon \mu \pi \eta a \sigma \imath \varsigma \mu \imath \nu \epsilon)$

In this way, AMPs have, integrating in either the viral envelope or the host cell membrane, basically four antiviral mechanisms: (a) viral envelope disruption; (b) viral receptors blocking; (c) preventing viral particles from entering host's cells by occupying specific cell receptors; (d) crossing the cell membrane and in the cytoplasm and organelles, cause changes in the gene expression profile of the host cells, helping them fight against viruses or block viral gene expression. It is believed that these effects are expected to happen because, whether cationic AMPs are able to effectively kill a complex microorganism (as seen, antibiotic resistant bacteria) with infectious agents like viruses this function may be achieved a lot easier. Thus, it is believed that, given their broad and diverse mechanism of action, like with bacteria, AMPs are designed to not be resisted by any sort of virus. Therefore, AMPs have a powerful mechanism against pathogens/diseases and now it is necessary to investigate, as most studies so far have only focused on propolis antioxidant substances, the possible special physical/structural action of propolis components perhaps mainly antimicrobial peptides (AMPs) -, which will be seen in the next section.

\section{Possible Existence of a Large Amount of Antimicrobial Peptides (AMPs) in Propolis}

\subsection{Main Direction of Research on Propolis}

The first scientific work with propolis was published in 1908, as mentioned before, including its chemical properties and composition. The differences in structure and function of the various propolis products do cloud the ability to recommend clinical and research direction. In general, health claims are likely to be isolated to a product generated from a particular geographical location. Despite the numerous health benefits found, until now, except for the American study quoted above that suggested the possibility, comparing the effects of propolis with AMPs (and maybe one or other sparse research); the studies about the medicinal 
properties of propolis and its compounds have been trying to find the mechanism of action of propolis, as the American study stated, believing that it is functional, attributed to one or some specific chemical compounds. There is limited research on the effects of propolis against pathogens, and how propolis may enhance bee products such as royal jelly and honey. The studies mostly isolate and try to find the compounds responsible for the effects of propolis, which is also complicated by the fact that, even though most propolis show efficacy against microbes and immunomodulatory effects, these researched compounds also varies according to the region that propolis is extracted and are always based on the local vegetation. Although propolis is a complex mixture, its biological activities are reported by these studies due to the presence of flavonoids - the largest group of naturally occurring phenolic compounds -, phenolic acids, and ethersmainly obtained from plant-derived substances. The main chemical components in propolis, studied mostly in terms of pharmacological activity, are pinocembrin, pinobanksin, caffeic acid phenetyl ester, artepillin C, cinnamic acid, p-coumaric acid, caffeic acid, ferulic acid, isoferulic acid, chrysin, galangin, kaempferol, and quercetin . The nature of these compounds - phenolic acids, terpenes, ethers and others - may be the reason why ethanol and other organic solvents are vastly utilized to extract the compounds of propolis, which, as will be discussed, may denature the protein compounds that are possibly the responsible for the disease fighting properties.

As an example of this trend to isolate and focus on only one chemical component, Artepillin C , a cinnamic acid derivative from Brazilian propolis, is the most illustrative. The substance is deeply researched, isolated and has various patents. Studies on it have been finding that the most important property are the strong antioxidants effects, demonstrating that Artepillin C exerted strong antioxidant activity and significantly inhibited the production of ROS, RNS, NO, and cytokines, and markedly blocked NF- $\chi \mathrm{B}$ expression in stimulated macrophages . Artepellin $\mathrm{C}$ also prevented oxidative damage dose-dependently, and suppressed lipid peroxidation in intestinal and hepatic cells . Moreover, lots of indirect cancer cells growth inhibition mechanisms of Artepillin $\mathrm{C}$ have been studied, demonstrating possible efficacy, such as in a study that suggested that both caffeic acid phenethyl ester and Artepellin C based propolis extracts are natural antiPAK1 remedies and could be among the first effective neurofibromatosis-associated tumors therapeutics available on the market. Another research found that Artepillin $\mathrm{C}$ showed high autophagy-inducing activity in prostate cancer cells . Lastly, a study already quoted above, also found that artepillin C, applied to human and murine malignant tumor cells in vitro and in vivo, exhibited a cytotoxic effect and the growth of tumor cells was clearly inhibited, with an increase in the ratio of CD4/CD8 T cells, and in the total number of helper $\mathrm{T}$ cells observed, suggesting that it activates the immune system and possesses direct antitumor activity .

Specifically regarding Artepillin C antimicrobial effects, besides its general possible immunomodulatory effects and the effects of propolis as a whole without the isolation of the component,almost no studies were found on the antimicrobial activity of the substance, with only one showing a sort of antibacterial effect with weak bacteriostatic activities and membrane blebbing. Thelack of studies and demonstrated antimicrobial effects are believed to be, at least, curious given that the main role of propolis in the hive is social immunity, mainly the protection against pathogens . In addition, the fact that bees do not consume propolis may be also considered - despite eating antioxidants from their food sources like pollen and nectar -, so they do not need the quoted researched effects directly in their organisms, they do not use the antioxidants collected for this, there might be something else .

Bees extract Artepellin C from a Brazilian plant called Baccharis dracunculifolia and, interestingly, antioxidants benefits found in propolis are similarly found in the hydroalcoholic extract from the plant leaves. The plant extract exert immunomodulatory action towards human neutrophils, modulating oxidative metabolism, degranulation, phagocytosis, microbial killing capacity and selectively modulating the effector functions, also inhibiting the activity of key enzymes and scavenging physiological oxidant species . Thus, it is speculated thathumans are totally capable of extracting these antioxidant compounds from vegetable sources and benefit of it. Therefore, in this line of thought and according to the focus of the current studies,propolis is being placed mainly as an antioxidant supplement used by humans 
that do not obtain the necessary amounts through food sources .

There are also many specific studies about cinnamic acid and derivatives such as the already seen Artepellin C, Baccharin and Drupanin. For example, one assay found that the combination of Baccharin and Drupanin in human colon cancer cells had an apoptosis-inductive effect; suggesting that the supplemental intake of these compounds found in propolis play a role in cancer prevention . Cinnamic acid derivatives, both isolated from plant material and synthesized, have been reported to have antibacterial, antiviral and antifungal properties and the activities are due to their acids, esters, amides, hydrazides and related derivatives . Regarding antibiofilm activity, in order to find alternatives to bacterial resistance, some studies are combining cinnamic acid derivatives with bionanotechnology engineered ultrashort peptides to obtain this activity . This combination is believed to suggest that these phenolic compounds are maybe just the vehicle and/or the enhancer for the active substances that are the peptides. Furthermore, in the same line of other phenolic compounds, cinnamic acid and its derivatives occur naturally in high levels in plant-based foods .

Other substances that attract attention of scientists and are encountered in propolis and other bee products are terpenes and their derivatives, which are large hydrocarbon groups and other plant-based compounds - also found, with acetates, in bee food sources like nectar and pollen . The disease fighting mechanism (antimicrobial and cytotoxic) seems to be also indirect and the effects are erratic and dosedependent, with most of the trials using in vitro cells or in vivo external use. Terpenes, for example, can be found in expressive amounts in coffee and cannabis. There are many other plant-derived constituents of propolis already studied by science that can be placed inside this suggested line of thought ofantioxidant indirect disease fighting properties - not direct effects like the ones from AMPs. All of these constituents found in propolis can be easily obtained through plant-based food sources as well. For this reason, in order to not extend the analysis of these compounds and divert from the main objective of the present study, they will not be analysed one by one.

Maybe all these phenolic compounds and other antioxidants from plants have another function in nature and are not the miracle cure of all diseases as researchers have been trying to exhaustively prove in the last years (although these antioxidants may somehow, if obtained together with other nutrients directly from a vegetable source, help in the prevention of diseases; but this would be a matter of another entire extensive study). Nowadays, experiments have been suggesting that antioxidants supplements or eating vast quantities of antioxidant rich food, actually, can be more harmful than helpful in the treatment of cancer, for example. A study found that antioxidant supplements differentially alter the intronic RNA expression of genes including several oncogenes . Furthermore, amongst the cadre of genes comprising the ROS system, the change in expression of introns was observed only in SOD gene. The differential intronic RNA expression after supplement treatments, especially the increase observed in oncogenes, suggests a possible effect on disease progression. Quantities of both ROS and natural antioxidants are higher in cancer cells - the paradoxically higher levels of antioxidants being a natural defense by cancer cells to keep their higher levels of oxidants in check, so growth can continue -; thus therapies that raise the levels of oxidants in cells may be beneficial, whereas those that act as antioxidants may further stimulate the cancer cells . These propositions and others studies introduce some clues that maybe the diseased organism does not need these huge amounts of "external" antioxidants given that the cells located at the disease site are, in normal conditions, capable of balancing naturally this ratio oxidants/antioxidants, and maybe there are other players involved .

This hypothesis is also sustained by the fact that, whether propolis is composed mostly by AMPs, they act on free-radicals through a much more complex antioxidant mechanism in comparison to all the antioxidants substances that propolis contains, not only through free radicals scavenging or simple inducing apoptosis. As known, excessive free radical generation, especially reactive oxygen species (ROS) that lead to oxidative stress in the biological system and generate the risk of chronic inflammation if ROS exceeds the antioxidant capacity, has been implicated in the pathogenesis and pathological conditions associated with several human inflammatory diseases. Therefore, in the normal resolution of inflammatory 
reactions, apoptosis is acknowledged to play a crucial role, while on the other hand, deregulation in the induction of apoptosis by enhanced ROS production could also result in excessive apoptosis identified in the pathogenesis of inflammatory diseases. Apparently, a careful balance must be maintained in this complex environment and antimicrobial peptides (AMPs) have been proposed as an excellent candidate, capable of playing prominent roles in maintaining this balance, because they can be considered to possess both pro-inflammatory and anti-inflammatory properties, suggesting that they are key players in the inflammatory microenvironment. So, this immunomodulatory role of AMPs may also be the reason why excessive antioxidant administration in some diseases is not necessary and can possibly make it worse, furthering the disease progression.

In order to illustrate an interesting finding about antioxidant compounds, phenolic lipids deserve consideration. They are found in propolis - in low amounts comparing to other components -, and arefound and absorbed by humans, for example, in vegetables with high fiber content such as cereal grains : rye, wheat, barley, and millet . Phenolic lipids have a strong amphiphilic character and are able to incorporate into erythrocytes and liposomal membranes, disturbing them (resemble, in part, AMPs). The interesting finding is the fact that the ability of phenolic lipids to inhibit bacterial, fungal, protozoan and parasite growth seems to depend on their interaction with proteins. These proteins, which may act with the other antioxidants in propolis as well, are believed to be AMPs, and this suggests that antioxidants may be only the vehicle/enhancer of the antimicrobial protein-derived mechanism of action, as will be further detailed.

Regarding the antimicrobial effects of antioxidants, a recent study tested $\mathbf{3 5}$ polyphenols on six foodborne pathogenic or food-spoiling bacterial strains and the effects of phenolic compounds found were highly heterogeneous, ranging from bacterial growth stimulation to antibacterial activity, and concluded that theantibacterial effect could not be clearly related to a class of polyphenols. With propolis, it was not different, in an essay testing six different common phenolic compounds (Coumaric acid, Kaempferol, Pinobanskin-3-acetate, Chrysin, Galangin, Kaempferide, and Artepillin C) applied directly against a variety of pathogens ( 6 different types of fungus and 10 different bacteria) it was found that the effect of each of these substances, when isolated from propolis, are weaker - more than two times, sometimes even three times or zero efficacy depending on the pathogen -than whole Brazilian green propolis ethanolic extract (BGP). The effects of the isolated phenolic compounds in the study were similar to the control, which was pure ethanolic alcohol - maybe because of their chemical similarities, remembering that ethanol only works if directly applied in a really high concentration and shows dubious and varied effectiveness against certain pathogens . Because the essayed fractions were not active, the authors suggested a synergistic effect of propolis constituents for the antimicrobial activity, andalso indicated that all microorganisms tested were susceptible to BGP - the effects of BGP were similar and sometimes superior than the ones obtained through the utilization of the controls Nystatin and Tetracycline, even though the BGP extraction method in the study was quite simple and the BGP was used in very low concentrations and in a reduced time frame.

In this way, antioxidants do not seem truly effective against diseases and pathogens, and their direct effects on pathogens may be compared to those of ethanolic alcohol or even kitchen vinegar - which can have, when directly applied, even more powerful effects against pathogens, in low concentrations, and even cause the death of cancer cells. Thus, if phenolic compounds effects are already weak when applied directly to the pathogen, what to think when they are utilized through peroral administration in which the absorption is low and excretion really high. Specifically referring to propolis phenolic compounds, a study pointed that factors believed to contribute to poor bioavailability of polyphenols include digestive instability, poor transcellular efflux in intestinal cells, and rapid metabolism and excretion. Since dietary polyphenols exist as esters, polymers or in glycosylated forms, they cannot be absorbed and must be hydrolysed by the intestinal enzymes or by the colonic microflora before absorption, which makes it variable and also the urinary excretion that appears to vary considerably between individuals, as will be detailed below in other section about propolis pharmacology. 
In order to strengthen the arguments, as a little observation, in relation to the defense mechanisms of plants, the research direction seems to be similar to the one adopted in propolis studies . Up to now, the knowledge about the way that plants protect, for example, their nectar from microorganisms is rather limited, with several reports focused on 'defensive chemicals', such as alkaloids and phenols , instead of in the protein contents that are what seems to show the real antimicrobial activity . Examples of bioactive peptides derived from internal sequences of proteins are known for decades, but the great majority of the findings appear to be fortuitous rather than the result of a deliberate and methodological-based enterprise. Plant antimicrobial peptides (AMPs) are a component of barrier defense system of plants, and have been isolated from roots, seeds, flowers, stems, and leaves of a wide variety of species and have activities towards phytopathogens, as well as against bacteria pathogenic to humans . Plant AMPs are grouped into several families and share general features such as positive charge, the presence of disulfide bonds (which stabilize the structure), and the mechanism of action targeting outer membrane structures .

Continuing, with regards to absorption and excretion, if phenolic compounds are compared, for example, with AMPs, the peptides elimination half-life seems to be higher, and some last for days . AMPs seem to also have better thermal stability and good water solubility, despite the fact that their protease stability can be low when ingested, but with some showing resistance to the hydrolysis of trypsin and pepsin. It is worth to already mention that, besides the digestion problem, it is believed that AMPs may adhere and cause gut bacteria death - and if propolis is, as studies have been showing, a strong antimicrobial substance, good or bad bacteria death may be the expected effect, especially if the effects stem from AMPs that have a physical/electrostatic mechanism of action, not biochemical/functional, without any selection between bacterial species. This effect on gut microbiota is not desirable in a sick organism; thus, it is believed that parenteral administration and direct or administration close to the site of the disease is necessary to keep the best efficacy of the peptides, as will be detailed below.

In this way, regardless of which compounds are responsible for propolis disease fighting properties, they seem to be showing stability, even in the digestive tract, because most studies in vivo use peroral administration and still show disease fighting efficacy of propolis. As stated and the studies are indirectly showing, propolis seems to have much more powerful effects than any of its plant derived compounds (phenolic compounds, terpenes, ethers and others), used isolated or together. So, despite studies having a different approach, there must be another strong mechanism involved in propolis infection/disease fighting properties .Propolis is so powerful that its extract, at low concentration $(10 \% \mathrm{w} / \mathrm{v}$,$) can be used effectively as insecticide against larvae of lesser wax moth,$ Achroia grisella. It is speculated that this sort of effect may not be provided only by phenolic compounds and shows the power of the application of the substance directly to the pathogen .

In this line of thought, although all studies about antioxidants in propolis seem reasonable, the believed hypothesis is that all these components in the substance are possibly acting only as natural necessary antioxidants. Maybe all these substances have only the role of preserving other most important components (possibly AMPs) that are inside the substance against oxidation from the air, sunlight, suspended particulate matter, humidity and other agents of oxidation, simple avoiding their deterioration and/or spoilage and the loss of their effects. Antioxidants may be also possible enhancers for AMPs effects mainly through $\mathrm{pH}$ regulation, as will be seen, simulating a disease site (this happens because the AMPs in propolis have to act "externally" and be "always ready", despite originally designed to act and be triggered inside an organism, using chemical signs and enhancers that occur in the disease site). Even in plants, this antioxidant mechanism of enhancement for AMPs seems to be plausible.

Propolis, as quoted above, consists of about $\mathbf{5 0 \%}$ resins , $30 \%$ waxes, $10 \%$ essential oils, $5 \%$ pollen, and $\mathbf{5 \%}$ various organic compounds, including flavonoids, phenylpropanoids, terpenes, stilbenes, lignans, coumarins, and their prenylated derivatives, with $>300$ different substances identified - other possibly most important unnoticed constituents will be discussed below in another section . The bulk of propolis are resins (possibly the AMPs abundant part), waxes and oils, which may also allow the 
substance to form a physical structure in the hive to seal cracks and crevices, providing thermal insulation, impermeabilization and stopping air flow. However, as seen, propolis most important function is immunity, i.e., protecting the hive against invaders and diseases, directly acting on intruders and sometimes "gluing/wrapping" them - also through volatile substances . Thus, it is suggested thatall these quoted organic components (5\%) already documented in studies so far, together with the others (except part of the resins, which may be the AMPs and will be discussed below), besides the physical necessity , are only the vehicle/enhancers and preservers of propolis active ingredients. AMPs are believed to be the active ingredients in propolis. It is speculated that, in propolis, the AMPs are possibly bound with less affinity to the other components and through electrostatic action "jump/glue" into the invaders, neutralizing them. As a study already evidenced that the peptide and not the resin is responsible for the antimicrobial activities of polymer-peptide conjugates. In conclusion, if all the documented phenolic compounds quoted are not the active ingredient, the powerful infection/disease fighting mechanism of propolis is possibly due to other components not yet studied, which will be detailed below.

\subsection{AMPs from Bees and from Plants and their Implications}

\subsubsection{AMPs from Bees and Implications}

As already said, bees and all invertebrates do not have an adaptive immune system and need AMPs for survival. This lack of adaptive defense is compensated by a further sophistication of the innate immune system, which is more ancient in evolutionary terms - bees are suggested to have existed for $\mathbf{1 2 0}$ million years - giving effective protection against microbial pathogens . It is believed that propolis, bees' social immunity tool, have evolved in the same way. The underlying principle, the humoral immune response of bees, involves transient de novo synthesis (memory formation) of a wide range of antimicrobial peptides (AMPs), which are synthesized in bees' fat body (liver-like organ) and secreted into their hemolymph (blood equivalent). They usually eliminate microbial pathogens by attacking their membranes and cell wall constituents and resistance to AMPs has so far not been observed , which makes them attractive candidates for the development of new antibiotics in human medicine . Thus,bees have been engineering, developing, and testing AMPs for millions of years .

In this way, regarding individual immunity, four families of AMPs (i.e.,apidaecins, abaecin, hymenoptaecin and defensins ) have been described in the honey bee and all of them are cationic peptides . A research in China with the Asiatic honeybee, Apis cerana F., in a comparison with the Western honeybee, Apis mellifera L., studied sequences of AMPs cDNA genes of both bees and found, in Apis cerana genes, coding of 7 different defensin peptides; 2 different abaecin peptides; 4 apidaecin peptides, and 13 different hymenoptaecin peptides ; i.e., about 26 different AMPs . For Apis mellifera, there were found genes encoding 3 defensin peptides, 2 abaecin peptides, 5 apidaecin peptides, and 1 hymenoptaecin peptide; however, as the study stated, it is likely that additional antimicrobial peptide and cDNA genes exist in the Western honeybee. Interestingly, the same study observed that detailed comparison of these four antimicrobial peptide gene families of the Asiatic honeybee with those of the Western honeybee revealed that there are many similarities in the quantity and amino acid components of peptides in the abaecin, defensin and apidaecin families . This is speculated to be the reason why, as already said above, allbees' genetically encoded defensive mechanism, individual or social like propolis, regardless of the origin, are really similar, which suggests that all propolis types are supposed to work all over the world .

As a great promise for exploitation by the pharmaceutical industry, several studies have been exploring the potential of these sorts of peptides and trying to synthesize them and use in the treatment of human diseases, especially to avoid bacterial resistance. However, despite the growing demand for antimicrobial peptides (AMPs) for clinical use as an alternative approach against antibiotic-resistant bacteria, themanufacture of AMPs relies on expensive, small-scale chemical methods . Regarding bee AMPs, various studies have been demonstrating that apidaecin , as a cationic, proline-rich antimicrobial peptide originally isolated from honeybees, exhibit highGram-negative activity by inhibiting bacterial protein translation, arresting bacterial terminating ribosomes with a unique mechanism of action . Also, abaecin, 
which among AMPs from the honeybee Apis mellifera, is considered a major broad-spectrum antibacterial proline-enriched cationic peptide, showed effectiveness against gram-positive bacteria and fungi, having antimicrobial activity against E. Coli and biotechnologicalpotential for the production of new antimicrobial drug, which acts against bacterial resistance to current drugs. It is worth to stress that the same study mentioned that abaecin sometimes needs to be combined with other AMPs to exhibit action. This combination necessity is lined to the statements that have been exposed in this work, believing that propolis action against diseases, which may be attributed to AMPs, as will be further discussed, depends mainly on the synergy of the antimicrobial peptides - despite other compounds may be sometimes useful as already speculated.

Proline-rich peptides, such bee apidaecins, represent a promising class of potential therapeutics to treat multiresistant infections because they inhibit bacterial protein translation by trapping release factors and also have a powerful post-antibiotic effect in vitro, usually 4-fold stronger than for conventional antibiotics, which helps to explain their high in vivo efficacy despite unfavourable pharmacokinetics. While most AMPs act by permeabilizing the bacterial membrane, the proline-rich antimicrobial peptides (PrAMPs) pass through membranes and inhibit bacterial growth by targeting fundamental intracellular processes, binding to the ribosome and interfering with the process of protein synthesis. An interesting fact to illustrate this potential is that researchers are trying to imitate Api137, a derivative of apidaecin, in order to makenew drugs that would kill bad bacteria using a similar mechanism of action. This is believed to possibly not work in the best form because of the lack of synergy with other AMPs, and also shows thatscience is trying to imitate a component that maybe bees produce in abundance and may "give" for "free" .

In this line, another research found that, in combination , abaecin enhanced the bactericidal effects ofhymenoptaecin . To understand these potentiating functional interactions, the study investigated their mechanisms of action and abaecin was found to reduce the minimal inhibitory concentration of hymenoptaecin and to interact with the bacterial chaperone DnaK (an evolutionarily conserved central organizer of the bacterial chaperone network) when the membrane was compromised by hymenoptaecin . It is the synergy of only 2 AMPs, but, as quoted above, at least 26 AMPs have been already found and studied in bees, with a possible unthinkable range of effects against diseases. AMPs combination are so powerful that, even AMPs from different origins can be combined and achieve synergy, like in a study that combined cecropin A, melittin and apidaecin (from insects), LL 19-27 and indolicidin (from mammals) and pexiganan (Pex) (synthesized AMP), concluding that they were mostly synergistic, with three-AMPs combinations displaying stronger synergism than two-AMPs combinations - The study also suggested synergism to be a common phenomenon in AMPs interaction and, additionally, stressed thatAMPs displayed a sharp increase in killing within a narrow dose range , contrasting with those of antibiotics . This also shows that these AMPs exchange between species in the case of the present study between humans and bees, also exposing the possible exchange of AMPs between bees and plants - is possible and may be beneficial. There are other examples of this brilliant synergy between AMPs, demonstrating their potential as a set and not as isolated compounds. So, the possible unique electrostatic/physical profile produced by the synergy of AMPs is believed to be what makes AMPs so powerful against infections/diseases. Despite the same cationic electrostatic mechanism of action, each peptide may have a different structural/physical form that produce specific effects after binding. All this also raises the question of why the researchers and the pharmaceutical industry have been trying to synthesize in laboratories these bees' (and other insects) AMPs and use them alone, exploring only one mechanism of action - especially while bees are showing the necessity of combined use; synthesizing them for free for millions of years, adding to their products and possibly making them available for humans in huge amounts.

The last family of bee's individual immunity antimicrobial peptides and still not addressed, defensins have a high level of polymorphism and exist as two peptides - defensin 1 and 2 , they are multiple-disulfide bonded (like AMPs from plantsquoted above), with a cationic charge and an amphipathic design. Defensin 1 is synthesized in bees' salivary glands and is responsible for social immunity ; defensin 
2 is synthesized by cells of the fat body and hemolymph and is responsible for individual immunity, with both showing a broad spectrum of antimicrobial action . Also, defensin-1, a regular but concentration variable factor found in honey and royal jelly, contributes to cutaneous wound closure by enhancing keratinocyte migration and matrix metalloproteinase-9 secretion. Although the full mechanism of action for bee defensin-1 has not been elucidated, defensin proteinsfrom other species have been shown to create a pore within the bacterial cell membrane, resulting in cell death . Therefore, as can be seen, defensin , only one of a big family, is a bioactive element composed by amino acids, engineered by bees andencountered in honey and royal jelly - together with several other antimicrobial peptides, as will be discussed. However, no studies were found about the existence of this sort of peptide in propolis. This is really odd given that the main role of propolis in the hive is immunity, defending it against pathogens and diseases.

Beyond individual immunity, several bioactive peptides (e.g., apamin and melittin) were identified in bee venom, and defensin1 (as cited, found in honey and royal jelly), actually, was originally isolated from royal jelly, and hence named royalisin . It is also known, besides defensin-1, that raw honey has small amounts of the same substances found in propolis, and it is possible that theantimicrobial properties of materials used and stored in combs (e.g., royal jelly, honey) are enhanced by the addition of propolis and some of the phenolic compounds in honey may be derived from propolis. This may be the reason for the presence of defensin and for the therapeutic properties of honey and is an indicator of the possible existence of AMPs in propolis. Another work onglycoproteins isolated from honey, similar to MRJP1, demonstrated rapid bactericidal activity of these glycoproteinsand that they possess suitable characteristics to be considered anovel antibacterial drug candidate. Thus, if these glycoproteins - found as a tiny portion of honey, but exerting strong antimicrobial effects - stem from propolis, it is also a strong clue for the power of these substances that may be present in huge amounts in propolis .

Referring to the antimicrobial activity of honey as well, another study found the lytic activity of the product - this is the only other trial that, besides the American one quoted above about propolis, suggested the existence of possible lytic activity in a bee product. The study found that the cell wall lytic bactericidal activity of MRJP1 glycoproteins (the antimicrobial peptides also found in royal jelly ) are directly correlated with the overall antibacterial activity of honey, suggesting that it is theactive principle responsible for this activity in honey. Thereof, it can be indirectly inferred that the strong antibacterial possible physical activity of propolis may stem from a mechanism that is similar to the one found about honey in the study quoted, and may be attributed to the existence of AMPs in the substance.

Lastly, still in relation to bees' other bioactive peptides besides those for individual immunity, royal jelly , given its medicinal properties, has been studied as well, with the analysis demonstrating that the main protein constituents are Major Royal Jelly Proteins (MRJPs 1 or royalactin and MRJP 2,3,4 and 5), and it also has royalisin, apisin, apisimin, apalbumin 1 (also in honey), jeleins 1,2, 3 and 4, but there are surprisingly few studies that address the biochemical characterization and functions of these components, and others yet to be studied protein and peptides. MRJP2 is indicated to have antimicrobial and antioxidants effects .Apisimin has demonstrated immunostimulatory properties . It is believed that the difficult with bee products is that there are several lines of research of their medicinal compounds and the amino acids, peptides and proteins seem to be the less explored , with sparse materials and an urgent need to thoroughly study and catalogue all the proteins and peptides and their possible effects on human health .

\subsubsection{AMPs from Plants and their Implications in Bees}

There are some studies documenting the use/consumption, by bees, of plant bioactive peptides - antimicrobial peptides (AMPs) as already stated . An essay with honey found that apisimin act as immunostimulatory in synergy with plant-derived arabinogalactan proteins (AGPs) also present in

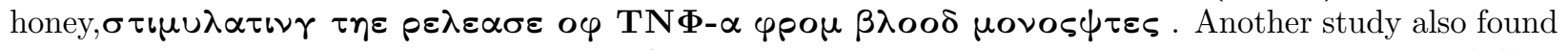
that the immunostimulatory activity of kanuka honey may be particularly dependent on AGPs 
derived from the nectar of kanuka flowers . Arabinogalactan proteins haveantimicrobial properties, and have amphiphilic natureas well . Bees consume and utilize, in their products, nectar (another plant exudate among various used by bees ), which is one more plant source of potent antimicrobial activity through proteins. This activity seems to be through the powerful mechanism ofribonuclease . Poplar Extrafloral Nectar, a kind of nectar that seems to attract bees, also has antimicrobial activity and this activity appeared to be associated with the protein moieties in the nectar. In this line, one form of social immunity in bee colonies is the collection of antimicrobial plant resins and their use in the nest architecture as propolis. So, it is believed that these bioactive peptides from plants possibly correspond to a considerable part of propolis given that a large amount of its compounds are plant-derived substances .

As can be seen, the studies quoted demonstrate that bees have the innate ability to use/consume and benefit from peptides/proteins from plants possibly because of their immunostimulatory and antimicrobial properties - and other effects that will be detailed below. This combined use of plant substances, if deeply studied and proven, is another extraordinary feature of bees, i.e., utilizing for their own defense the AMPs "borrowed" from the local plants, which tend to be previously and strongly adapted to the local pathogens. One point that is intriguing in the studies pointed so far are the findings about the presence of antimicrobial substances from plants and bees, in a small portion - possibly to not damage bees' gut -, in products that are consumed by bees such as honey, royal jelly, nectar and others. This certainly raises a question about what would be the amount of these peptides in the product not consumed and that is designed solely for defense, i.e., propolis - the fact that bees do not consume propolis is also believed to be an indicator that propolis has strong effects and is not naturally designed for the digestive tract .

Besides the effects on social immunity, there are other possible effects of the consumption of AMPs from plants by bees. These amino acids/peptides may exert effects on bees' biological processes. For example, bees prefer proline-rich nectars, and some plants offer proline-rich nectars as a mechanism to attract visiting pollinators. Proline, beyond the possible antimicrobial properties, is required by bees for egg laying and increasing the size of their hypopharyngeal gland acini. Among the processes stimulated by plants, this increase of hypopharyngeal glands is believed to be really important because it is required to generate, together with the mandibular salivary glands and maybe others, bee products, such as protein rich royal jelly and possibly propolis .

Thereby, this consumption/use of plant peptides and the consequent stimulation of glands may raise the production of propolis as it is produced by bees through mixing the secretions of their hypopharyngeal glands with the digested product of resins collected from leaves, flowers of plants, trees, and certain barks. Also, theseantimicrobial resins added in propolis possibly have AMPs and thus this suggests the possible extensive use of the peptides from plants in propolis . It is believed that there is a possiblevirtuous circle between bees and plants : when AMPs from plants are abundant in the environment, the protection/stimulation provided by them is large and the synthesis/secretion of bees' own AMPs grows; this, in consequence, increases even more the social immunity, which allows bees to invest less in individual immunity, raise overall production/survival rates, and hence forage more AMPs from plants, and so on. In conclusion, all this suggests and reinforce the strong mutualism between bees and plants, i.e., bees may consume and extensively use in their products (especially propolis) , given the antimicrobial/disease fighting properties, AMPs already synthesized from plants, and the consumption of these special peptides may also stimulate, among bees' other important metabolic and biological functions, the synthesis of bees' own AMPs in the hypopharyngeal and mandibular glands and possible secretion in bee products (especially propolis).

It is worth emphasizing once again that this stimulation of bees' glands furthered by amino acids and peptides from plants - a possible sign of a favorable environment -, as will be detailed in the next section, may be extremely important for the production of propolis . This is because bees possibly use these glands to produce their own AMPs and perhaps secrete these special peptides in 
propolis - as seen, propolis has secretions from hypopharyngeal glands, thus suggesting a possible massive production of AMPs in these glands and secretion in propolis . Beyond the ones from hypopharyngeal glands, there is also a clue for the use of secretions from bees' mandibular glands in propolis - glands that possibly are also stimulated by plants bioactive peptides and, as said, may work together with hypopharyngeal in the production of propolis. For example, glucose oxidase, which is produced in themandibular glands of bees, has been found in propolis .

Ultimately, as a parentheses about a curious finding regarding the possible secretion of AMPs from the mandibular glands in propolis, which will be vastly discussed below, it is worth to mention the peptide defensin 1 . The bee peptide, which has a broad spectrum of antimicrobial action, as shown above, is synthesized in the mandibular salivary glands of bees and is responsible for social immunity . Defensin 1 is regularly found in honey and royal jelly and is also reported to besecreted by bees' hypopharyngeal glands - suggesting again that these glands may work together with the salivary glands in the immunity role. If defensin 1 , despite no reports of its existence in propolis so far, is designed for social immunity and secreted in honey and royal jelly; what to say about the possible secretion of AMPs from the glands in question in propolis and hencethe possible abundant presence of defensin 1 in the substance that, more than needing to be preserved, has the specific role of defending the hive. It is not just rhetorical or speculative, but a strong and simply logical question that needs to be investigated.

\subsection{Bees' Possible Mechanism of Social Immunity by means of AMPs}

In regards to AMPs production and possible role in bees' social immunity, a study with Apis mellifera, the most common of honey bees worldwide, revealed that forager bees, different than nurses that do not, highly express immune and detoxification genes in tissues associated with nectar processing , suggesting that forager role and tissue-specific expression of AMPs and immune and detoxification enzymes may contribute to defense against microorganisms and xenobiotic compounds acquired while foraging . Interestingly, in contrast, the expression ofantimicrobial transcripts in the midgut did not differ between nurses and foragers, i.e., the difference was observed only in the hypopharyngeal and mandibular glands. This is supposed to indicate that this expression of immune genes is not directly implicated in individual defense but as a way to raise the social immunity of the hive, especially because foragers are the responsible for the production of propolis that is the strongest defense of the colony - and, as quoted, in the nest propolis allows bees to invest less in their individual immune systems, preserving their immune system gene expression and energy in order to raise their colony productivity and survival rates. Furthermore, nectar already has its natural defense that stems from the possible AMPs that it contains - AGPs and protein moieties as the studies quoted above found -, so, it isunlikely that all nectar consumed by bees may have significant amount of microorganisms that could cause such an immune reaction. It is also not believed that bees' natural source of food - which, as seen in the previous section, may also stimulate bees' biological processes - may cause any sort of allergy (the same argument will be more deeply detailed for pollen below and the reasoning is the same for nectar). Therefore, it is suggested that this expression may be attributed to the protein content of nectar that stimulates the production of AMPs that are secreted in bee products including and mainly in propolis, as it is going to be further demonstrated.

The study about the increase in gene expression also stated thatextremely high expression levels of antimicrobial peptide genes in the hypopharyngeal gland were found and levels this high are rare and restricted to specialized tissues. That is, in other words, cells have to divide their efforts between housekeeping and specialized functions related to their differentiated role in the organism for many tissues, this balance should be far from parity such that cells exert most of their effort on tissue-specific functions. Extremely high expression and patterns of AMP coexpression with nectar conversion enzymes indicate that the specialized function of the hypopharyngeal gland may also include the production of antimicrobial peptides to preserve foraged resources , but further experimental work is necessary to test this hypothesis . It is suggested that the production 
of these peptides, given their nature, is not only to preserve foraged resources, but has the primary objective of protection of the whole hive(including the foraged resources and all the colony members), and this protection is possibly achieved by adding these AMPs in the synthesis of propolis ; the substance responsible for social immunity. Also, the study about the increase in gene expression pointed that the expression of antimicrobial peptides and effectors of immune response in glands involved in social interactions among bees suggest thatsocial insects, including many pollinators, may employ a wider range of mechanisms against environmentally acquired microorganisms and xenobiotics than previously appreciated. In this way, it is speculated that bees' hypopharyngeal and mandibular glands possibly are specialized social immunity production tissues. These tissues are stimulated by protein rich content in food sources (especially proline), as will be detailed below, with various different AMPs expression, synthesizing and secreting them to become social immunity most powerful tool of the living organism that the hive is.

Another analysis about bee genes, in Apis mellifera L,demonstrated that the expression of the genes for some carbohydrate-metabolizing enzymes, which are needed to process nectar into honey in the hypopharyngeal gland, is associated with the age-dependent role change of the worker . So, this finding also support the supposed fact that maybe the increase in the expression of immune genes in tissues that process nectar - and also produces propolis - are only age/function related and a small difference between foragers (need the high expression to produce social immunity) and nurses (do not need the expression given they are not involved with social immunity until becoming adults, but only benefit of the social immunity produced by foragers). So, the increase in the gene expression and production of AMPs in foragers may not be, mostly, reactive, but functional and natural and they are used in the production of social immunity (AMPS are added to honey, royal jelly and perhaps especially to propolis). This argument, as will be exposed below, is also supported by the fact that, in a high protein diet, bee's AMPs expression increases, which possibly make themquantitatively improve the social immunity protection.

In this line of thought, an interesting research demonstrated the significance of feeding bees with different protein diets, as well as the importance of pollen nutrition for bee immunity. It showed that the expression levels of the apidaecins and abaecin genes were affected by the protein diets, comparing to less or no protein diets, and different expression levels of these two antimicrobial peptides were found, and they augmented in a protein rich diet. This leads to the believed hypothesis that bees, in a high or normal protein supply situation, indicating a favorable environment and conditions for population growth, possibly use these peptides through mandibular/hypopharyngeal glands secretion to quantitatively improvesocial immunity rather than using the peptides on individual immunity . Strong indicators for this hypothesis are the studies already cited demonstrating that bees prefer proline-rich nectars , andsome plants offer proline-rich nectars as a mechanism to attract visiting pollinators ; and that proline-rich nectar increases egg laying and the size of bees' hypopharyngeal gland acini , which, as seen, is responsible for AMPs production. It is worth remembering the fact that apidaecins are proline-rich peptides and represent a promising class of potential therapeutics to treat multiresistant infections. So, still following the same reasoning, these protein/proline-rich food effects on bees; their AMPs production in a protein rich-diet and possible social immunity improvement; and the prolinebased composition of apidaecins are all also clues for the existence of large amount of proline-rich peptides, with strong antimicrobial effects, in the social immunity tool that is propolis .

It is worth to note that, in regards to the research about feeding bees rich protein diets previously quoted; it also brought the hypothesis that pollen alone could contain certain biomolecules that could activate immune reactions, which alternatively could explain the variable gene expression and production of antimicrobial peptides . Although plausible, this hypothesis is not believed to be entirely true. This stems from the findings exposed in the study about the possible age-dependent role change of the worker bee mentioned before, showing that the difference between the genes of workers and nurses may be only age related (perhaps specialization) and not just food activated immune reactions. Beyond this, the other study already exposed above, suggested the possible specialization of hypopharyngeal glands in the production of AMPs - with differences in immune genes expression observed only in forager glands 
and not in nurses. The same study found that the expression of antimicrobial transcripts in the midgut did not differ between nurses and foragers, in spite of the microorganisms encountered by both in the hive stored sources of food ; demonstrating, therefore, that immune reactions to food sources may not occur. Following this line, as shown and also as a known fact, nectar and pollen are processed in the same tissues involved with AMPs production, i.e., they may directly stimulate these tissues - mainly through proline - and not cause immune reactions .

Thereby, in part, it is suggested that the argument in the study about feeding bees rich protein diets is reasonable, because thebiomolecules in pollen may really activate immune genes.However, it is believed that this activation is not an immune reaction. Actually, the activation is caused, as seen, maybe by the components in pollen (proline and other amino acids and peptides), which are believed to be favorable signaling from the environment. These components directly stimulates the immune genes involved and make them increase the production of AMPs, which, in consequence, quantitatively improve bees' social immunity protection through propolis - this signaling from nature through proline, as discussed, induces a range of other benefic effects on the colony such as lessening bees' individual immunity expression, increasing egg laying, etc. Another clue for the exposed arguments are the notorious millions of years of mutualism between bees and plants and, given that pollen is one of the main sources of food for bees, it may not be an "allergenic". As a final observation, these findings possibly suggest that a rich protein diet could be a way to enhance social immunity and, consequently,the quality of the propolis produced and bee products made.

Notwithstanding the fact that all propolis antioxidants properties and mechanisms, as indicated above, were vastly studied by science so far and the claims may be valid, after all the findings and suggestions pointed throughout the present study, the proposed hypothesis, which will be tested with experimental evidence in this work, is: bees,regarding social immunity, synthesize many different powerful cationic AMPs (there are more than 30 genetically encoded AMPs already known, with 120 million years of evolution, mostly proline/arginine-rich and with broad spectrum antibacterial and disease fighting synergistic actions) inside their immunity production specialized tissues(hypopharyngeal and mandibular glands) based on the amino acids/proteins contents of their diets (especially proline that is able to increase the immune gene expression of these tissues),secreting and mixing the produced ones with AMPs extracted from plants (AGPs, "resins"), and adding them in all their products (primarily propolis ). This possible extraordinary state-of-the-art immunity technique, stimulated by favorable signs of nature, may be intended to raise the social immunity of the living organism that is the hive, preserving, as the studies exposed above demonstrated, bees' individual immune system gene expression and energy in order to raise their colony productivity and survival rates - and the technique is also believed to allow bees to preserve foraged resources that are stored inside the hive and the eggs and larvae that do not have defenses. In this way, it is suggested thatpropolis, which may be mostly composed by AMPs, is possibly supposed to imitate bees' individual immune system in a stronger manner as it is the protection of the entire organism that the hive is. That is, it is possible to claim, in a simple manner, thatpropolis contains a large amount of AMPs.

In order to sum all up, until now, in the scientific literature, it was found that the strongest evidence of the broad use of AMPs in bee products are the studies already mentioned above findingseveral different AMPs, from bees and plants, in Royal Jelly and Honey - remembering that one trial demonstrated thatthe peptides, and not other compounds, are the responsible for the antimicrobial activity in honey, with possible lytic action; hence suggesting a mechanism of action that is the same of cationic AMPs. As clues of the use of AMPs in bee products, there are a few studies that generically state that propolis has bee secretions and antimicrobial resins from plants; however, with no details of the composition or possible mechanism of action. Beyond these, some indicators of this possible ample use of AMPs are the findings in the trials quoted about bees' gene expression - suggesting a possible mechanism of social immunity production. What causes perplexity is the lack of investigation on the possible use of AMPs by bees specifically in propolis, which is the main tool of social immunity in the hive . However, as will be seen in the next section, astonishing clues of the possible existence of a 
large amount of AMPs in propolis can be easily found in some studies produced so far .

\subsection{Consistent Clues of the Existence of AMPs in Propolis}

As seen, propolis is a resinous substance variously composed of chewed tree buds mixed with pollen collected by bees andenzymes secreted by them. It is produced by honeybees throughmixing the secretions of their hypopharyngeal glands with the digested product of resins collected from leaves, flowers of plants, trees, and certain barks, and is mostly consisted of about50\% resins and $30 \%$ waxes. The resinous compounds from plants (possibly with significant amounts of AMPs) and the secretions of bees (possibly mostly AMPs) were already discussed above, but the bulk of this $50 \%$ mass containing mostly "resins"should be investigated more deeply . Bees, as exposed, has a preference for the amino acid proline and search for proline-rich nectars and pollens, with some plants offering proline-rich nectars as a mechanism to attract visiting pollinators . Proline is required by honey bees for egg laying and increasing the size of their hypopharyngeal gland acini, and the amino acid is the main part of several bee AMPs (e.g., apidaecin and abaecin, with strong antimicrobial activity). So, if propolis has AMPs as its main constituents, it must contain a generous amount of proline and/or other amino acids and derivatives .

Actually, the focus on propolis plant-derived antioxidants has been so expressive in the last decades that there is practically no scientific investigation about propolis amino acid/protein compounds. In 1996, there was a study in Brazil qualifying, quantifying and analysing the probable origin of all amino acids present in propolis resin from 4 different regions of the country. The study stated that, although many of the constituents of propolis have been identified and their biological properties investigated, only a few reports describe the amino acid content. And continued suggesting that the data obtained indicated that the amino acids from propolis may becollected by bees from oil and resinous substances from plants surfaces such as leaves of dicotyledonous plants and (or) pollen, and it can also be assumed that amino acids derived from the metabolism of the bees are introduced during formation of propolis .

In 2016, there was another research, which also quoted, as main sources, the study above made in Brazil and another one made in 1986 - which will be discussed below -, showing the interdependence of the few studies existing in the area. The 2016 study stressed again that the polyphenol content of propolis has received a lot of attention due to the benign biological properties noted in the chemical composition studies; however, there are very limited studiesabout other chemical components that may contribute to the therapeutic properties of propolis. In this way, the study, in a simple approach, investigated the amino acid and vitamin composition of propolis, suggesting that they may have therapeutic properties .

Even though no advances were done so far, the most interesting study was done more than 30 years ago, in 1986, and this fact allows inferringthe absolute scarcity of studies focused in the amino acid compounds of propolis. The work in 1986 surprisingly found that propolis contains, among other biochemical constituents, a variety of free amino acids and their total concentration in propolis is over $40 \% \mathrm{w} / \mathrm{w}$, and thatarginine and proline constitutes over $50 \%$ of the crude acid extract. This finding leads to the inevitable question of why more than $40 \%$ of propolis composition, which means most of the "resins" on it, was never deeply investigated. Since there is no waste in nature, why all these amino acids are present in propolis; they are too much to be only a structural reason. This huge amount of amino acids may have another important functionconsidering that bees do not consume propolis . Therefore, it is not possible to believe that, in order to produce propolis, bees are merely wasting these precious components - especially given the fact that all this feedstock firstly passes through a specialized tissue that possibly fabricates protein derived substances for social immunity, as already discussed above.

Which is also surprising is that $50 \%$ of the amino acids (aprox.20\% of propolis and half of the "resins") arearginine and proline. Both are well known to be the main components of cationic antimicrobial peptides (AMPs) that have a broad spectrum of antimicrobial activity and can kill bacteria, 
fungi, viruses and protozoan parasites . Bees' proline-rich peptides such as apidaecins represent a promising class of potential therapeutics to treat multiresistant infections. The finding of proline in propolis is also totally in accordance with the discussed preference of bees for proline-rich foods and the stimulation that the amino acid exerts on them, and maybe this is why bees need so much proline in their food sources. In consequence this huge amount of amino acids found is the strongest and most consistent indicator of the possible existence of a large amount of AMPs in propolis .

Therefore, the existence of a large amount of amino acids (mainly those types used for cationic peptides synthesis, which have promising antimicrobial effects); added to the facts pointed throughout the present work; hypotheses raised; and the medicinal effects of propolis (similar to AMPs) that science has been sparsely proving; all leave, in theory, little doubt that propolis infection/disease fighting mechanism stems mostly from the fact that the substance possibly has a large amount of AMPs. Now that it is demonstrated that propolis is a substance rich in amino acids, what is necessary isto find a way to physically evidence the hypothesis that these amino acids, actually, are the main constituents of a large amount of cationic antimicrobial peptides (AMPs) existing in propolis, and also observe the extension and the power of this mechanism . This is what will be tried next.

\section{Experimental Evidence of the Existence of a Large Amount of AMPs in Propolis}

In order to evidence all the possible extraordinary claims from the previous section, some trials were done and described in here. Despite the scarcity of time due to the outbreak of COVID-19 and the limitations in the development of experiments in an independent research, out of a professional setting, it is worth mentioning that the validity of the evidence obtained during the hypothesis testing was not compromised in any moment. Through experiments that can be easily reproduced, extraordinary evidence was possibly revealed - "extraordinary claims require extraordinary evidence".

\subsection{Summary}

Hypothesis: propolis has a large amount of cationic AMPs; i.e., propolis has, given the significant hydrophobic residues of the water-soluble cationic peptides that abound in it, amphipathic/surfactant character and its therapeutic mechanism of action is physical/structural, through electrostatic force; thus, in order to produce effects, the cationic peptides in propolis bind, by attraction, to anionic moieties of the organism/agent with which the substance interacts, and generate a cascade of phenomena.

Methods and Materials: given the circumstances, a fast, cheap, and reliable method had to be designed. As AMPs have common features like cationic charge, hydrophobic residues with a consequent amphipathic character and water-solubility ; if propolis really has a large amount of cationic AMPs, then it must easily show these features. In this way, this physical mechanism would be seen even with the naked eye. So, the behavior and effects of propolis and its possible cationic peptides (AMPs) were compared with those of liquid dishwashing soap/detergent (anionic). For this, six types of experiments were designed with propolis, always comparing it with dishwashing detergent.

Results: in all six experiments developed (and other two additional ones) propolis behavior and effects demonstrated strong cationic and amphipathic/surfactant character (even in a low concentration), which certainly stems from water-soluble compounds (AMPs) that also showed the existence of hydrophobic residues; propolis sometimes presented an opposite behavior when compared to the anionic detergent, and a different mechanism of action.

Conclusion/Discussion: the results of the experiments and all the evidence existing in the scientific literature firmly support the hypothesis and it cannot be rejected. Beyond the initial claim, the effects seem to be brutal. The results also suggest that propolis has strong antimicrobial and disease fighting properties andis designed to not be resisted by any pathogen, especially if applied directly to the infection/disease site in the necessary amount. So, the demonstrated hypothesis of a large amount of cationic AMPs in propolis and all its implications, besides a possible alternative to be used now against SARS-CoV-2, should be extensively explored by science. 


\subsection{Hypothesis}

As previously quoted, the hypothesis of the present study is that bees, based on the amino acids/proteins contents of their diets, synthesize several different powerful cationic AMPs inside their immunity production specialized tissues; these AMPs are secreted and mixed with AMPs extracted from plants, and they are all added to propolis. Consequently, propolis is a possible AMPs-rich substance and its therapeutic effects stem from them. So, considering the studies demonstrating the existence of a large amount of amino acids in propolis (mainly those types used for cationic antimicrobial peptides synthesis ) and the fact that AMPs have common features - which are their overall cationic charge, a significant fraction of hydrophobic residues and an ensuingamphipathic character ; and are water-soluble ; it was possible to develop the reasoning. That is, if these four main features are present in all AMPs, then propolis (or a great part of it) must present these four features to be considered, in fact, a substance that have a large amount of AMPs - or, more precisely, a substance in which a large amount of amino acids existing in it are, indeed, the main constituents of a large amount of antimicrobial peptides (AMPs).

In addition, if the AMPs that possibly exist in propolis, as stated, have to: be bioactive (i.e., must present the exposed features/electrostatic mechanism of action, which phenolic compounds, wax, pollen oils and other compounds in propolis do not present); be encountered in a large amount (following the hypothesis that bees use a lot of them in the social immunity tool; and in order to produce effects that can be easily seen); be amino acid derived (amino acids mostly used for cationic peptides synthesis, as seen, are the greatest part of propolis); and, be water-soluble (besides the amino acids, which are the bulk of propolis, most of other components, as exposed, are not water-soluble); there is no doubt that these AMPs will be constituted by the large amount of amino acids in propolis, which will surely be the elements responsible for the potential behavior/effects produced. In this way, if the four characteristics of AMPs are observed in the tests with propolis, it will be physically demonstrated that the large amount of amino acids found in the substance, actually, are the main constituents of a large amount of AMPs existing in propolis .

Therefore, if propolis (or a great part of it) presents the four characteristics/effects of AMPs, then it will be demonstrated that the amino acids in the substance form indeed bioactive compounds (the character that will be observed cannot be attributed only to isolated amino acids, they must be combined to produce the effects) andhence claiming that propolis has a large amount of amino acids - which is already documented - will be the same of claiming that propolis has a large amount of cationic AMPs (for the sake of technical accuracy, despite a sort of pleonasm, as AMPs in general, as shown, are commonly cationic and bee AMPs are all cationic, to highlight this feature, the possible AMPs in propolis, from now on, will be called cationic AMPs ). Besides this, as anatural and obvious consequence and also as the citedAmerican study already demonstrated and suggested, if the existence of a large amount of AMPs and its effects are demonstrated in propolis, it will have demonstrated as well the physical/electrostatic/structural therapeutic mechanism of action of AMPs - as seen in the description of the mechanism of action of AMPs above ; to produce effects, they work through electrostatic force, generating a series of phenomena in which one leads to the next, like a cascade.

In this way, in order to keep in line with the background of the study and facilitate the design, observation and conclusion of the experiments, a detailed hypothesis was formulated: propolis has a large amount of cationic AMPs; i.e., propolis has, given thesignificant hydrophobic residues of the water-soluble cationic peptides that abound in it, amphipathic/surfactant character and its therapeutic mechanism of action is physical/structural, through electrostatic force; thus, in order to produce effects, the cationic peptides in propolis bind, by attraction, to anionic moieties of the organism/agent with which the substance interacts, and generate a cascade of phenomena.

It is worth mentioning that, which also coincides with the few existing studies on amino acids in the substance, after a deep research, no studies were found about the possible existence of AMPs in propolis. In relation to propolis amphipathic/surfactant (surface active), detergent/soap-like, or emulsifier effects, no studies were 
found as well. So, the present work seems to be the first to specifically research the existence of AMPs in propolis and these effects that may be derived from them. As the main initial clues for the present investigation, it is convenient to remember, in the American study quoted above, the demonstration of the existence of a physical/structural mechanism of action of propolis and the suggestion that propolis ethanolic extract may be compared to lytic peptides or Cationic Antimicrobial Peptides ; and, in the study already cited on the lytic activity of the peptide MRJP1 in honey, the demonstration of a lytic effect of a peptide from bees .

\subsection{Methods and Materials}

Given the scarcity of time due to the spread velocity of COVID-19, the issue was how to develop a fast, cheap and reliable method to test the presented hypothesis efficiently, demonstrating the real benefits that propolis may bring to the unfair fight against this terrible disease. Thus, as already stated, AMPs have common features, which are their overall cationic charge ; a significant fraction ofhydrophobic residues and an ensuing amphipathic character ; and are water-soluble. So, whether propolis really has a large amount of cationic AMPs, then it must easily show thesecationic detergent-like features and the cationic AMPs must be water-soluble. In this way, this physical mechanism of action would be seen even through a "macro" and simple experiment, with the naked eye and independent of special instruments.

With these AMPs characteristics in mind it would be possible to compare the behavior and effects of the possible cationic AMPs in propolis with a very common and easily found component, i.e., liquid dishwashing soap/detergent. This sort of detergents has amphipathic behavior and effects basically by the use of anionic surfactants such as linear alkylbenzene sulfonate (LAS), as the one used in the experiment, or Ammonium lauryl sulfate (ALS), and others. Given the presence of ions in the dishwashing detergent and also the opposite charge and hypothetical opposite behavior and effects in some situations - which would allow differentiating from propolis suggested cationic AMPs behavior and effects - it was possible to design some experiments to identify and measure the power of the proposed hypothesis.

Therefore, six types of experiments were designed with propolis, always comparing it with dishwashing detergent:

a) breaking of the surface tension of water : dried and grinded oregano leaves were dropped in a soup dish filled with water, and the substances to test were dropped in the center of it; observing if they were capable to break the surface tension of the water and push the oregano to the corners of the dish.

b) emulsification of olive oil and water : in a container with water, olive oil was added to the surface and then the substances to test added and stirred, observing if they were able to emulsify/mix the oil with water (in 5 hours). Also, in this case, to test the effects in low temperatures, ice cream was made in the same way; but stirring sometimes during the freezing process and observing if a creamy texture was achieved.

c) emulsification of coconut oil and water : in a container with water, coconut oil (saturated fat) was added to the surface and then the substances to test added and stirred, observing if they were able to emulsify/mix the oil with water (in 5 hours).

d) removal of fat in hand-washing: fat from coconut oil was spread in clean hands and then they were washed with the substances to test, observing if the substances were able to remove all fat from the hands.

e) observation of cationic or anionic behavior : in a tea-cup filled with water, olive oil was dropped in the surface of water, and drops of the substances to test were placed in the center. The rapid change in the surface tension and behavior of the oil were observed (breaking of the tension and displacement of the oil to the center or corners) in order to identify if the substances were predominantly either cationic or anionic. Also, an additional test was carried out by mixing propolis with the anionic detergent in equal amounts; then, similarly, drops of the mixture were placed in a cup with water and oil, and the effects on the surface tension and oil behavior observed.

f) precipitation on salt : in a container filled with water, salt (sodium chloride) was added until a little part 
of it precipitated in the bottom. Then, drops of the substances to test were added and the precipitation or not, in the bottom, was observed in order to try to identify the ionic profile of the substances - i.e., e.g., if cationic, with some parts possibly binding to the negatively charged salt chloride ions and precipitating in the bottom. Some tests with the precipitated matter obtained were done as well (no pictures were taken).

The materials utilized were regular kitchen utensils (dishes, containers and cups); propolis aqueous (PAE) and ethanolic extracts11Apis Flora brand and as a control the brand Qualymel from Qualynutri, both aqueous and ethanolic Green propolis extracts (11\% w/v of dry propolis). (PEE) (a different brand was also used for both as a control and obtained the same results, so, it will not be documented); dishwashing detergent22Ypê dishwashing detergent (linear alkylbenzene sulfonate - LAS); double filtered water (regular filter and gravity filter, to reduce the mineral content); tap water for the hand washing experiment; grinded oregano leaves; olive and coconut oils33Gallo and the coconut oil Copra.; regular refined kitchen salt44Cisne, sodium chloride w/ iodine.; and 70\% ethyl alcohol. Given the simplicity and the possibility of easy reproduction of the experiments, the exact amounts of the materials utilized were not measured. All experiments were conducted in a domestic kitchen with an average temperature of $26 \mathrm{degC}$ and an altitude of $500 \mathrm{~m}$. The atmospheric pressure was not measured. As controls, water and alcohol (due to the aqueous and ethanolic extracts of propolis) were used.

\subsection{Results}

The results were summarized in the following table and documented through the pictures below:

\begin{tabular}{|c|c|c|c|c|c|}
\hline & PAE & $\mathrm{PEE}$ & DETERGENT & WATER & ALCOHOL \\
\hline $\begin{array}{l}\text { Breaking of } \\
\text { the surface } \\
\text { tension of } \\
\text { water }\end{array}$ & $\begin{array}{l}\text { Strong (Fig. } \\
\text { 1) }\end{array}$ & $\begin{array}{l}\text { Strong (Fig. } \\
\text { 2) }\end{array}$ & $\begin{array}{l}\text { Strong (Fig. } \\
\text { 3) }\end{array}$ & None & None \\
\hline $\begin{array}{l}\text { Emulsification of } \\
\text { olive oil and } \\
\text { water }\end{array}$ & $\begin{array}{l}\text { Strong (Fig. 4) } \\
{ }^{*} \text { creamy ice } \\
\text { cream (Fig. 7) }\end{array}$ & $\begin{array}{l}\text { Strong (Fig. 5) } \\
{ }^{*} \text { creamy ice } \\
\text { cream (Fig. 8) }\end{array}$ & $\begin{array}{l}\text { Strong (Fig. 6) } \\
\text { *brittle ice } \\
\text { cream (Fig. 9) }\end{array}$ & None & None \\
\hline $\begin{array}{l}\text { Emulsification } \\
\text { of coconut oil } \\
\text { and water }\end{array}$ & $\begin{array}{l}\text { Strong (a } \\
\text { larger amount } \\
\text { was needed in } \\
\text { comparison to } \\
\text { the one used } \\
\text { in olive oil) } \\
\text { (Fig. 10) }\end{array}$ & $\begin{array}{l}\text { Strong (a } \\
\text { larger amount } \\
\text { was needed in } \\
\text { comparison to } \\
\text { the one used } \\
\text { in olive oil) } \\
\text { (no picture) }\end{array}$ & $\begin{array}{l}\text { Strong (a } \\
\text { larger amount } \\
\text { was needed in } \\
\text { comparison to } \\
\text { the one used } \\
\text { in olive oil) } \\
\text { (Fig. 11) }\end{array}$ & None & None \\
\hline $\begin{array}{l}\text { Removal of fat } \\
\text { in } \\
\text { hand-washing }\end{array}$ & $\begin{array}{l}\text { Strong (no } \\
\text { pictures) }\end{array}$ & $\begin{array}{l}\text { Moderate (no } \\
\text { pictures) }\end{array}$ & $\begin{array}{l}\text { Moderate (no } \\
\text { pictures) }\end{array}$ & None & Weak to none \\
\hline $\begin{array}{l}\text { Observation of } \\
\text { cationic or } \\
\text { anionic behavior }\end{array}$ & $\begin{array}{l}\text { Strong cationic } \\
\text { (displacement to } \\
\text { the center) (Fig. } \\
\text { 12) }\end{array}$ & $\begin{array}{l}\text { Strong cationic } \\
\text { (displacement to } \\
\text { the center) (no } \\
\text { pictures) }\end{array}$ & $\begin{array}{l}\text { Strong anionic } \\
\text { (displacement to } \\
\text { the corners) } \\
\text { (Fig. 13) }\end{array}$ & $\begin{array}{l}\text { None (Fig. } 14, \\
\text { to show the oil } \\
\text { position before } \\
\text { adding the } \\
\text { substances) }\end{array}$ & None \\
\hline $\begin{array}{l}\text { Precipitation on } \\
\text { salt }\end{array}$ & $\begin{array}{l}\text { High (possible to } \\
\text { separate the } \\
\text { precipitated } \\
\text { PAE) (Fig. } 15 \\
\text { and Fig. 16) }\end{array}$ & $\begin{array}{l}\text { Low (alcohol } \\
\text { possibly } \\
\text { disturbed) (no } \\
\text { pictures) }\end{array}$ & None (Fig. 17) & None & None \\
\hline
\end{tabular}




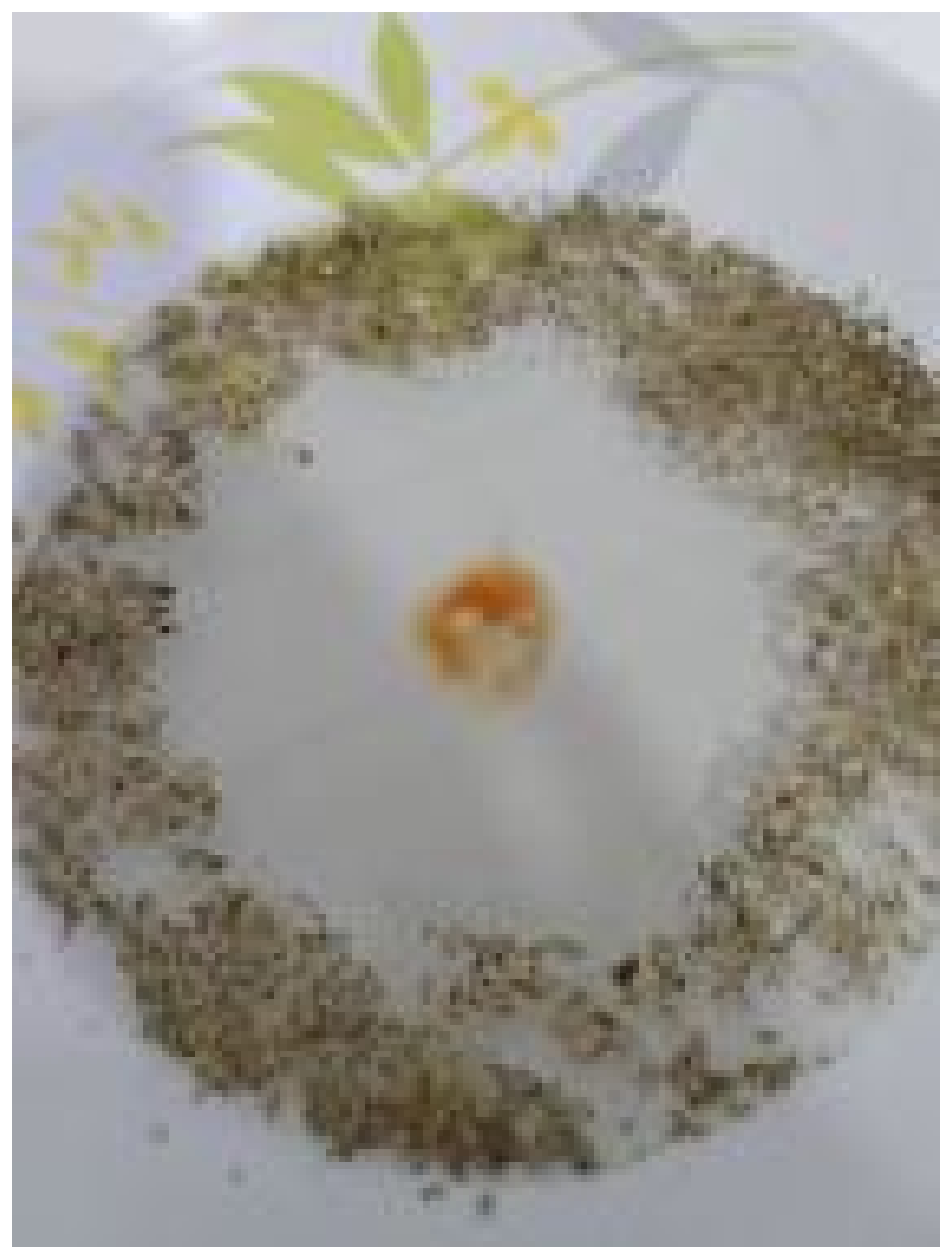




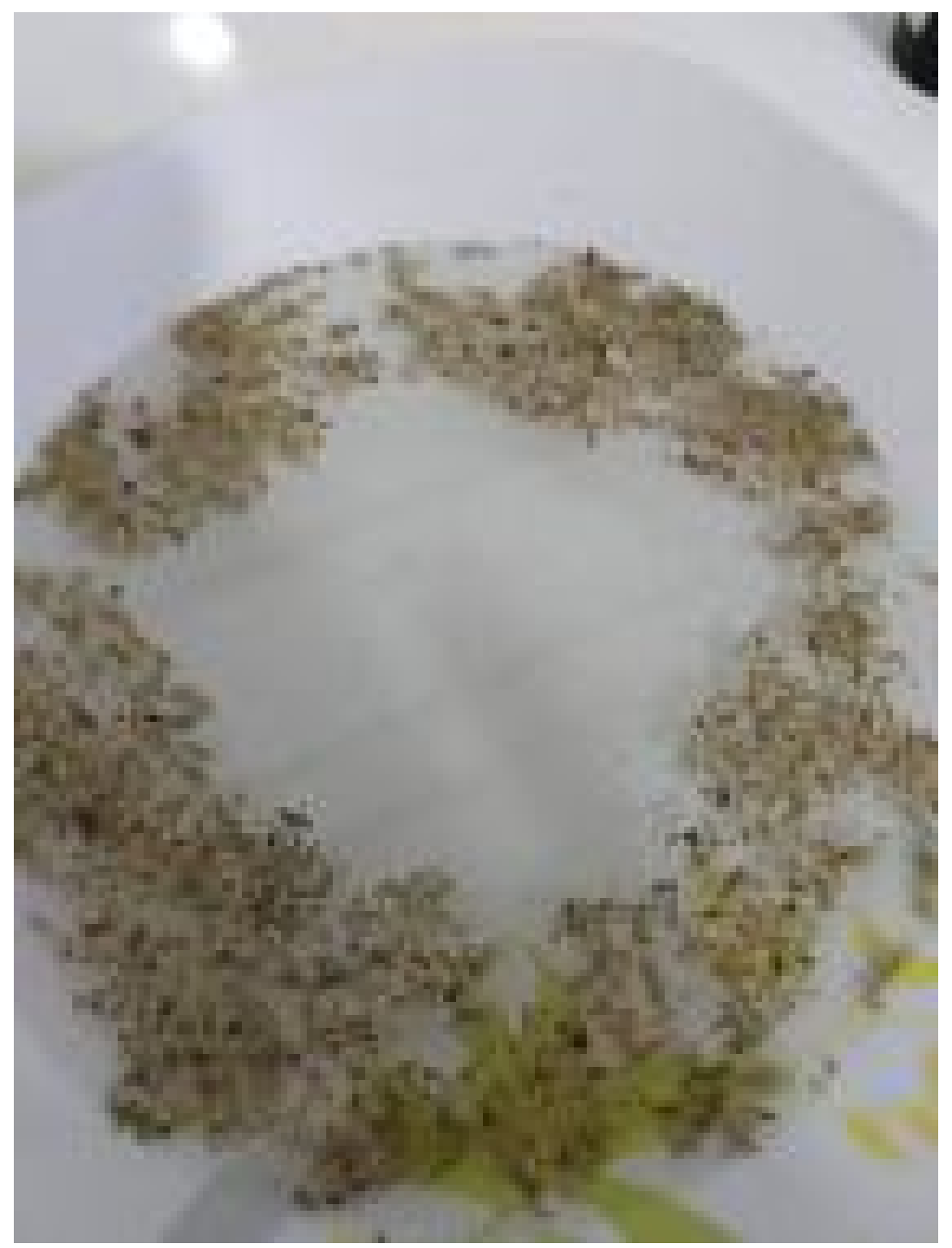




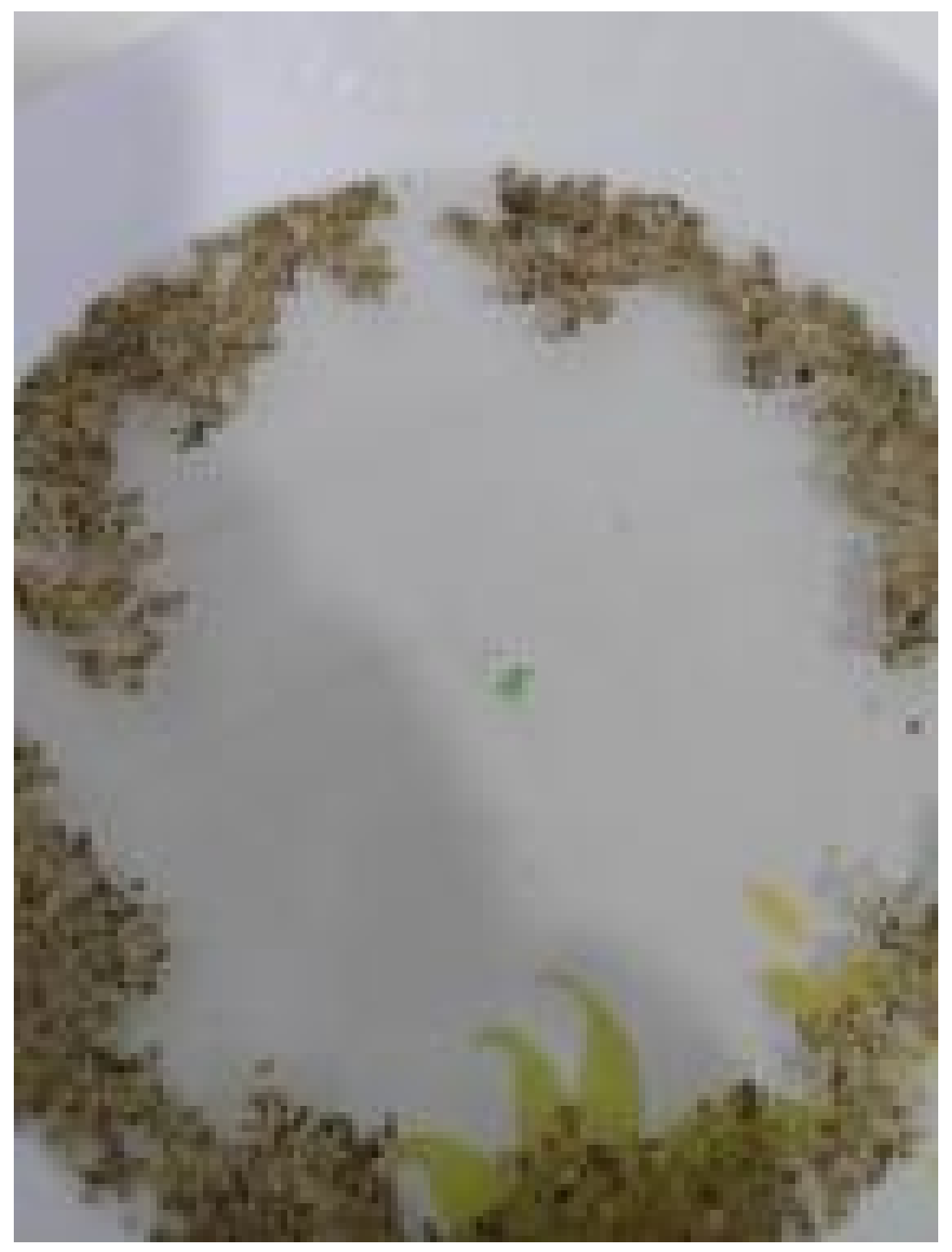

Fig. 1Fig.2Fig. 3 


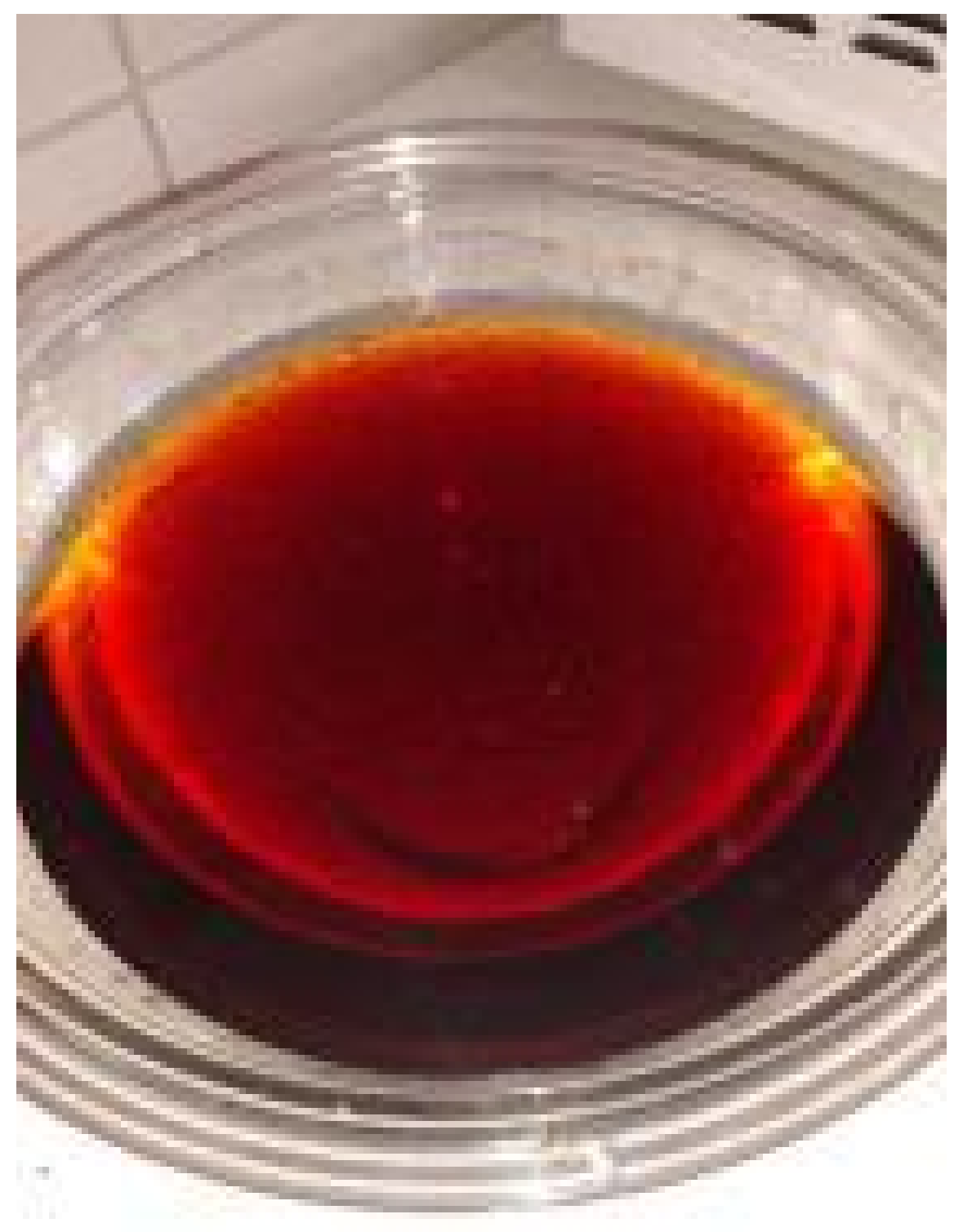




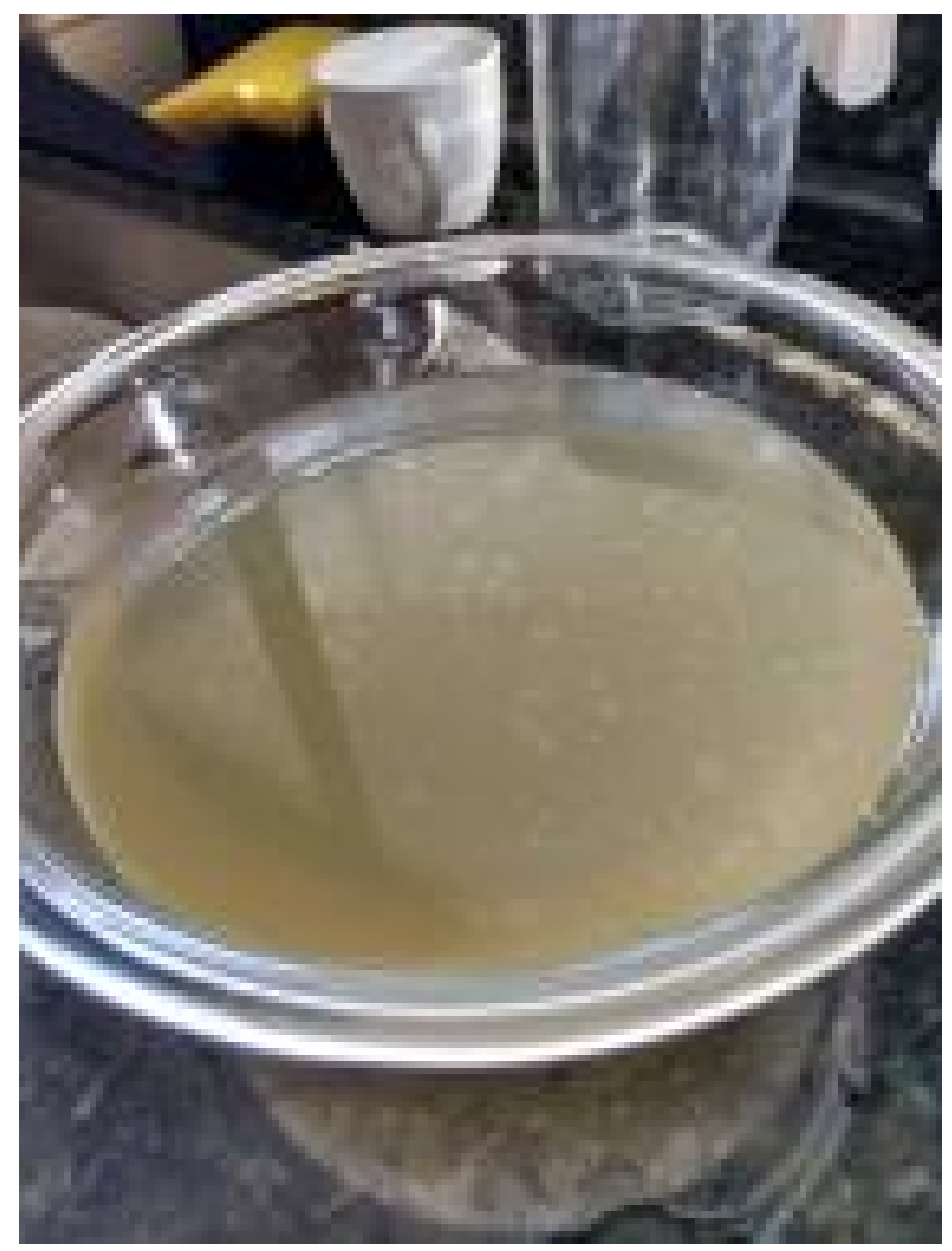




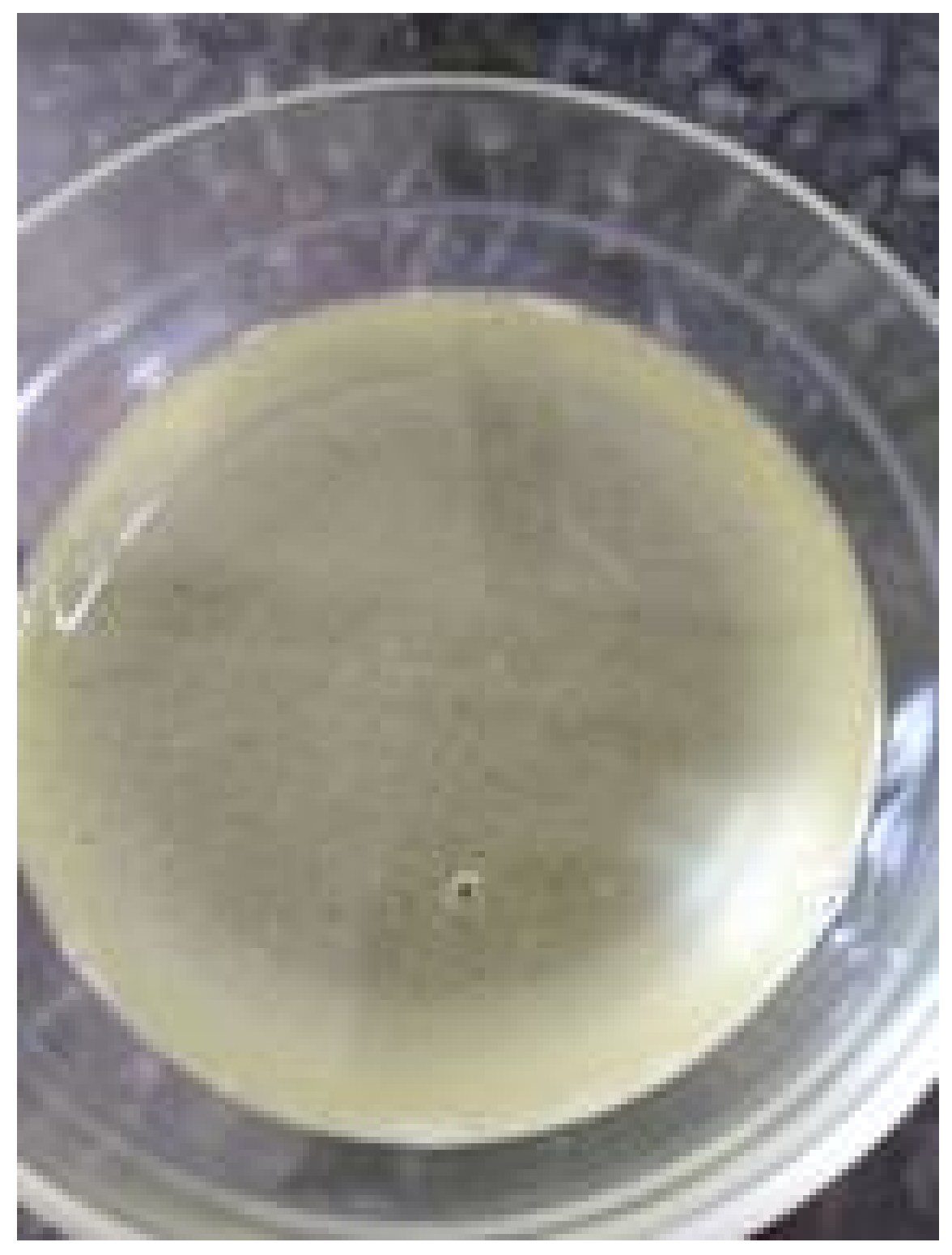

Fig. 4Fig. 5

Fig. 6 


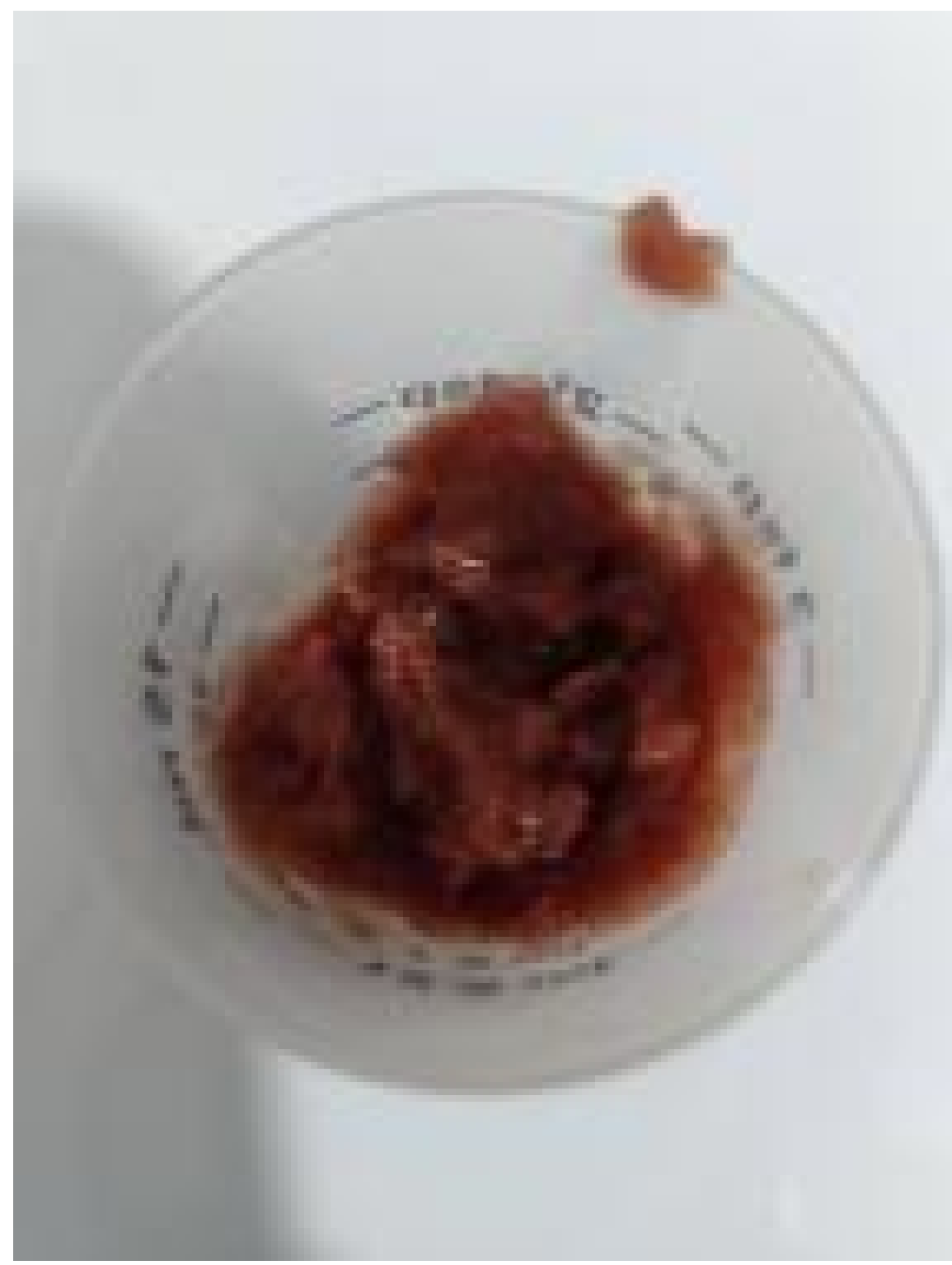




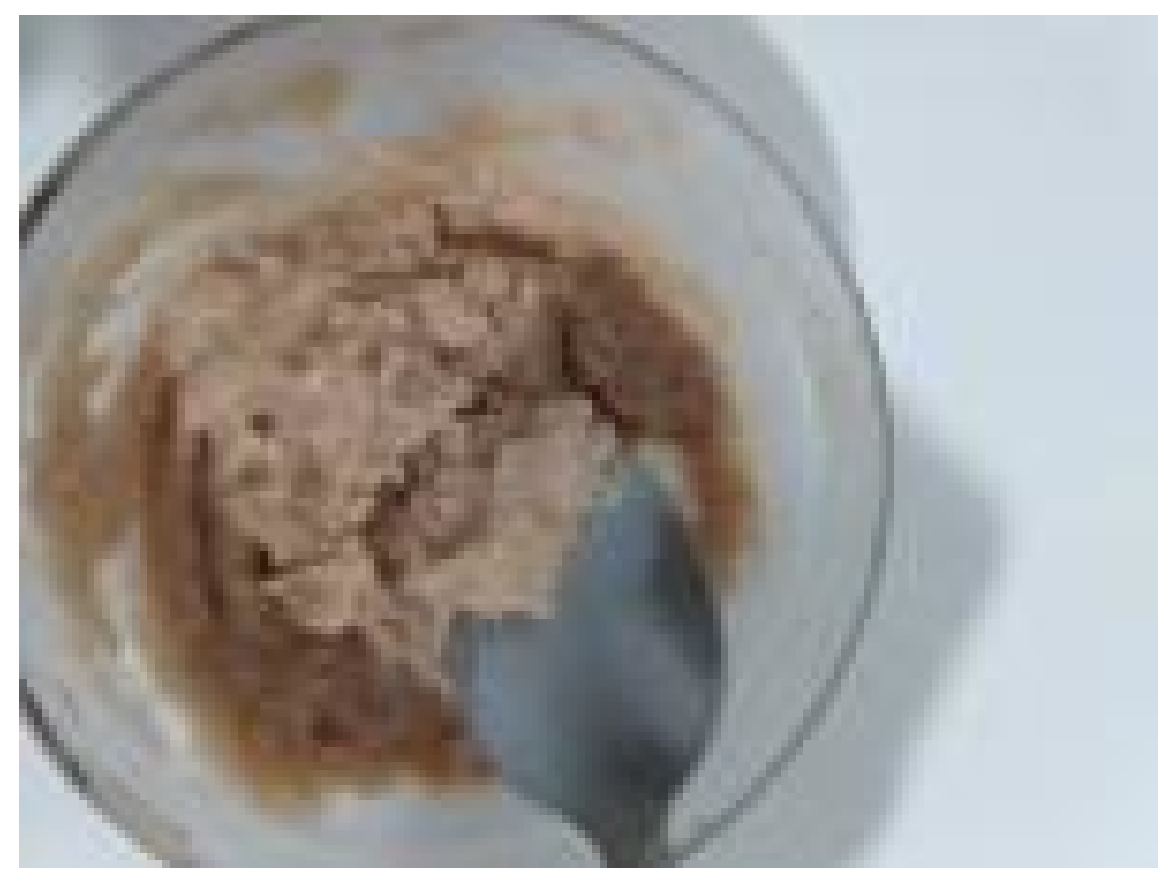




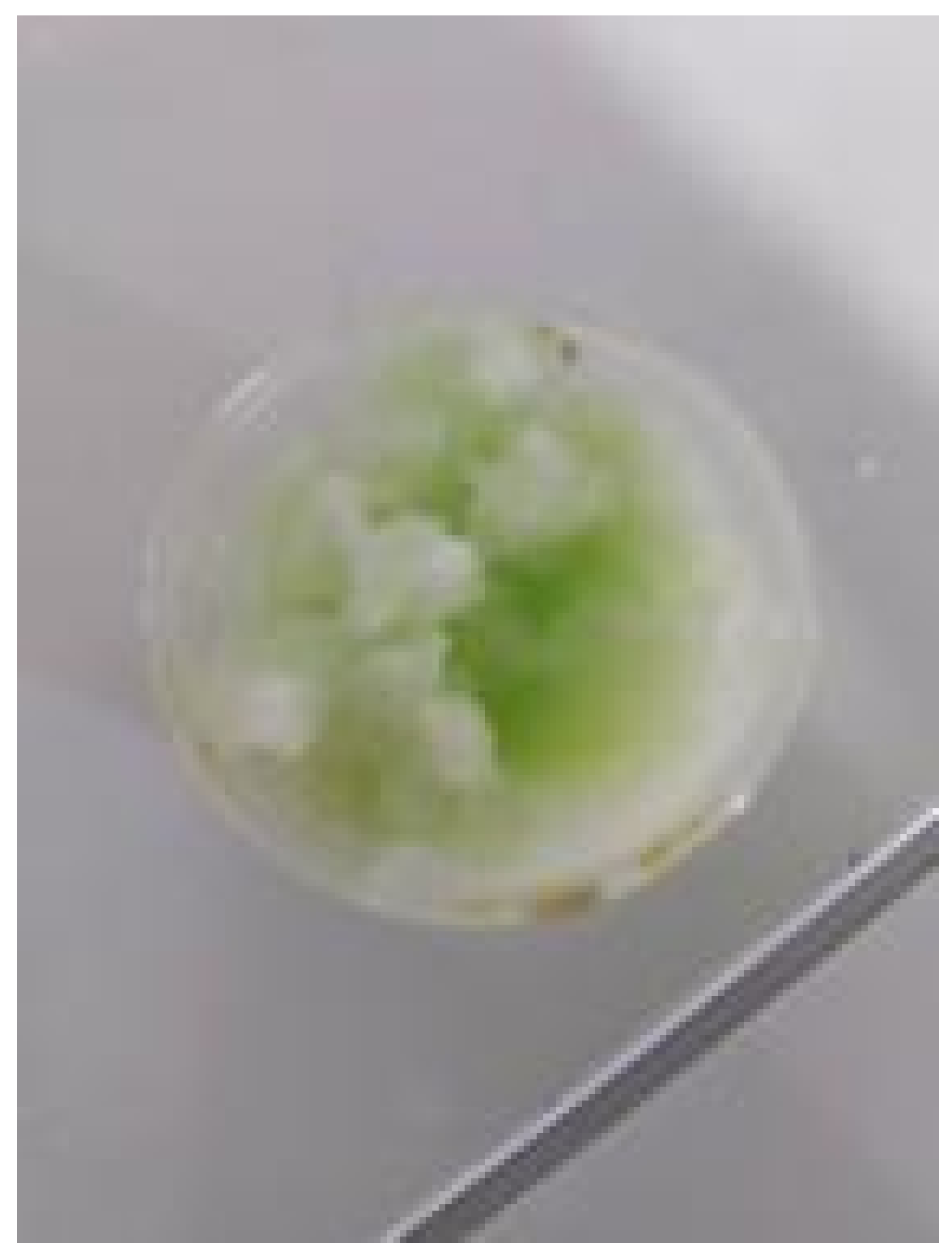

Fig. 7Fig. 8

Fig. 9 


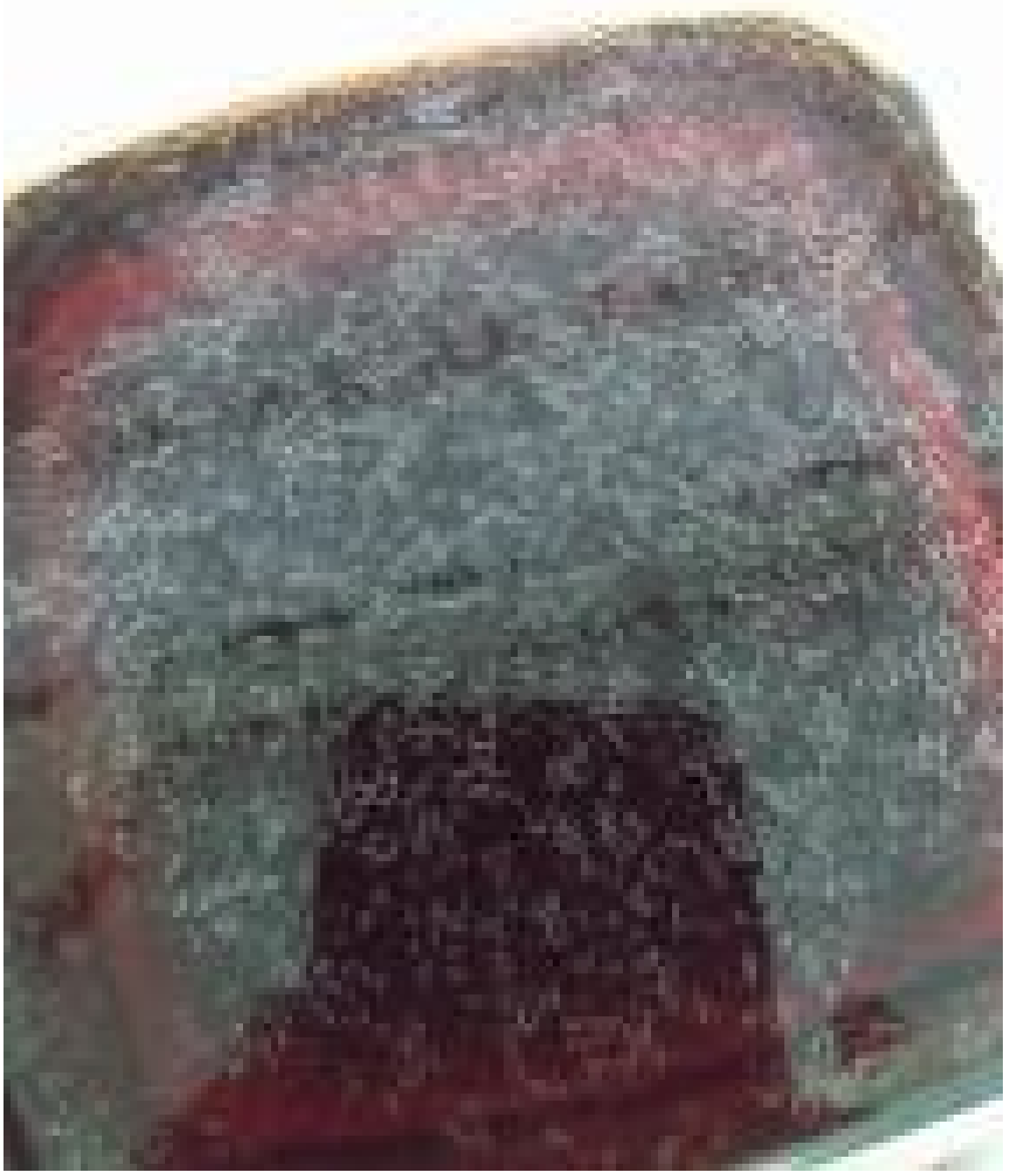




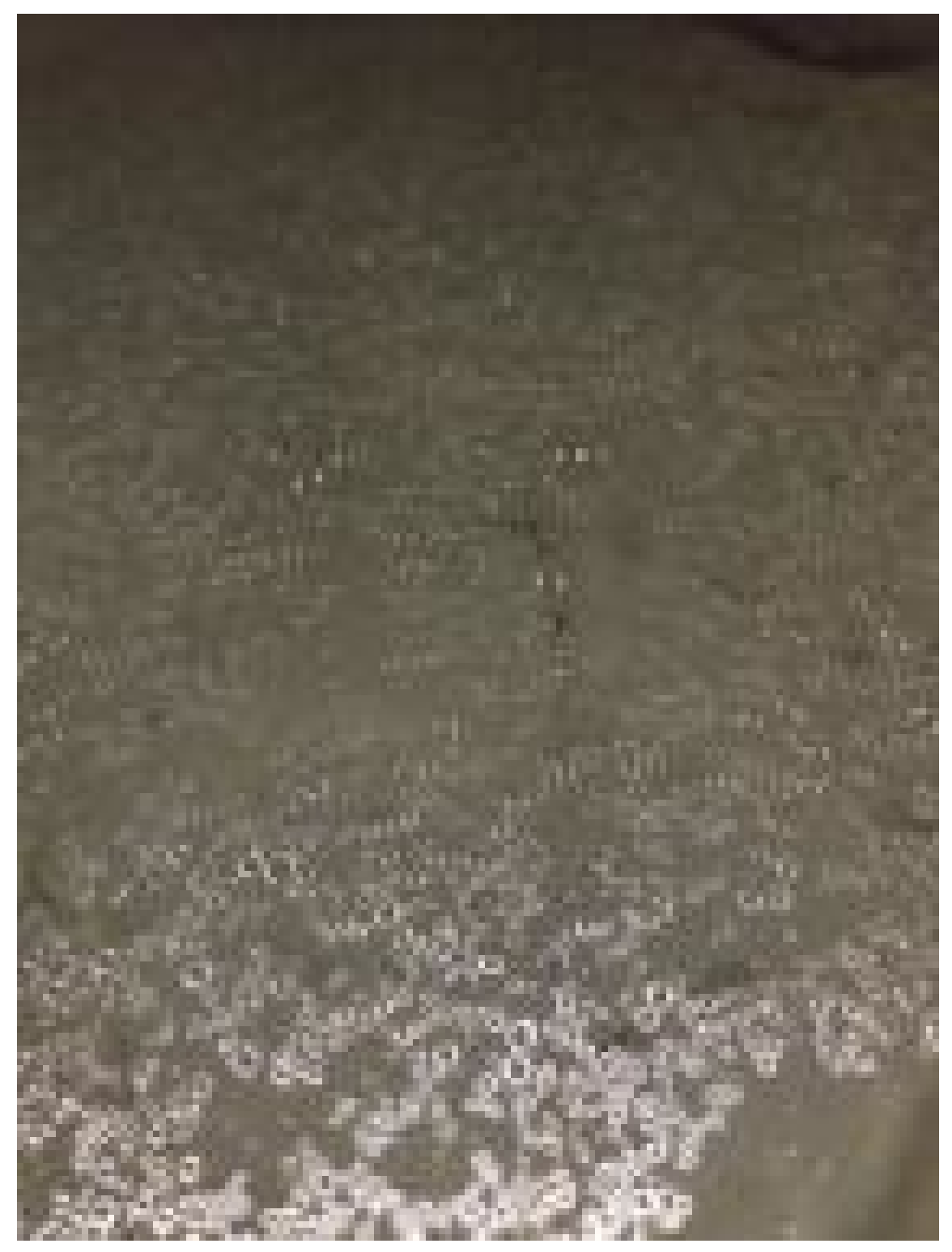

Fig. 10Fig. 11 


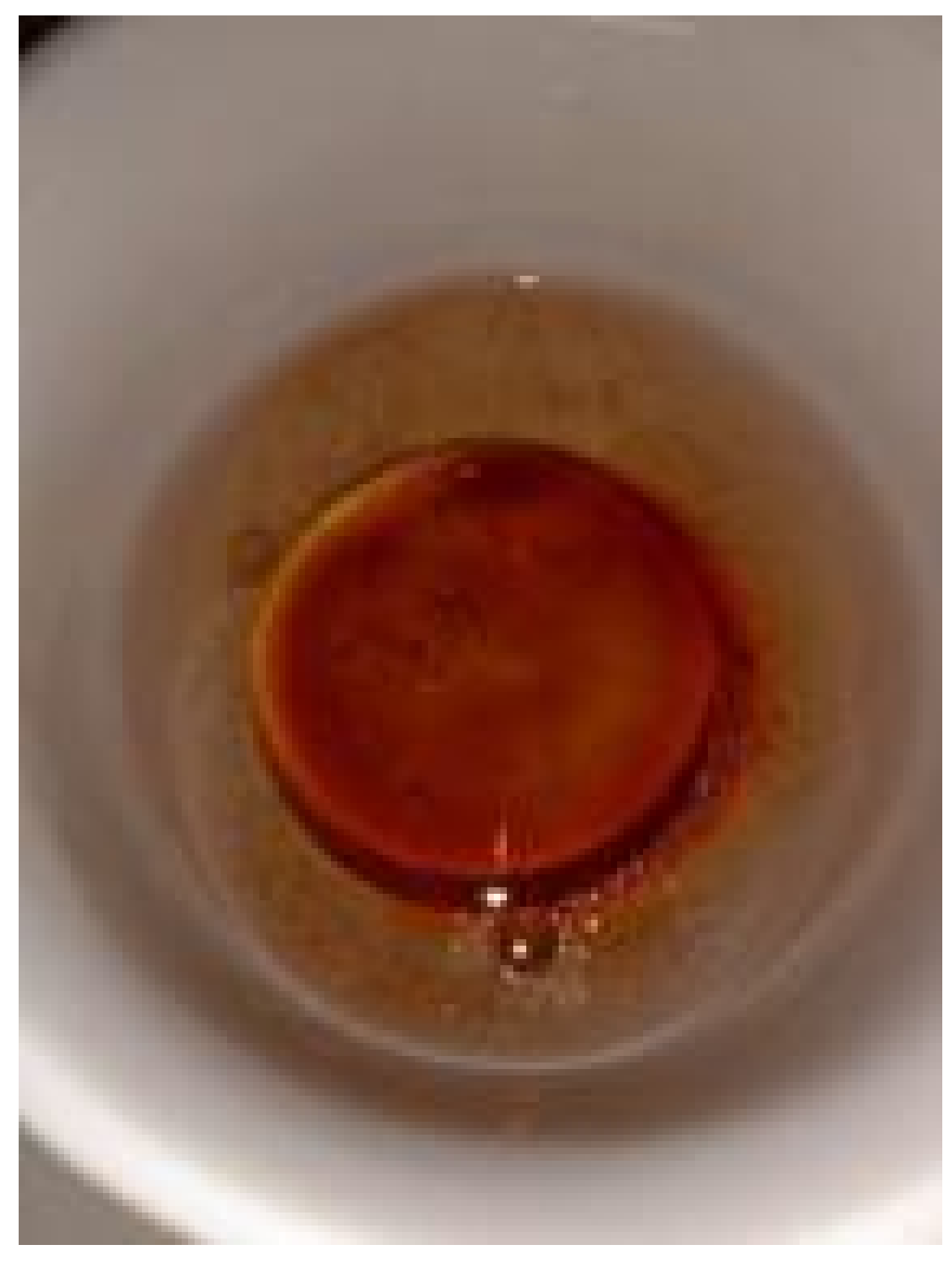




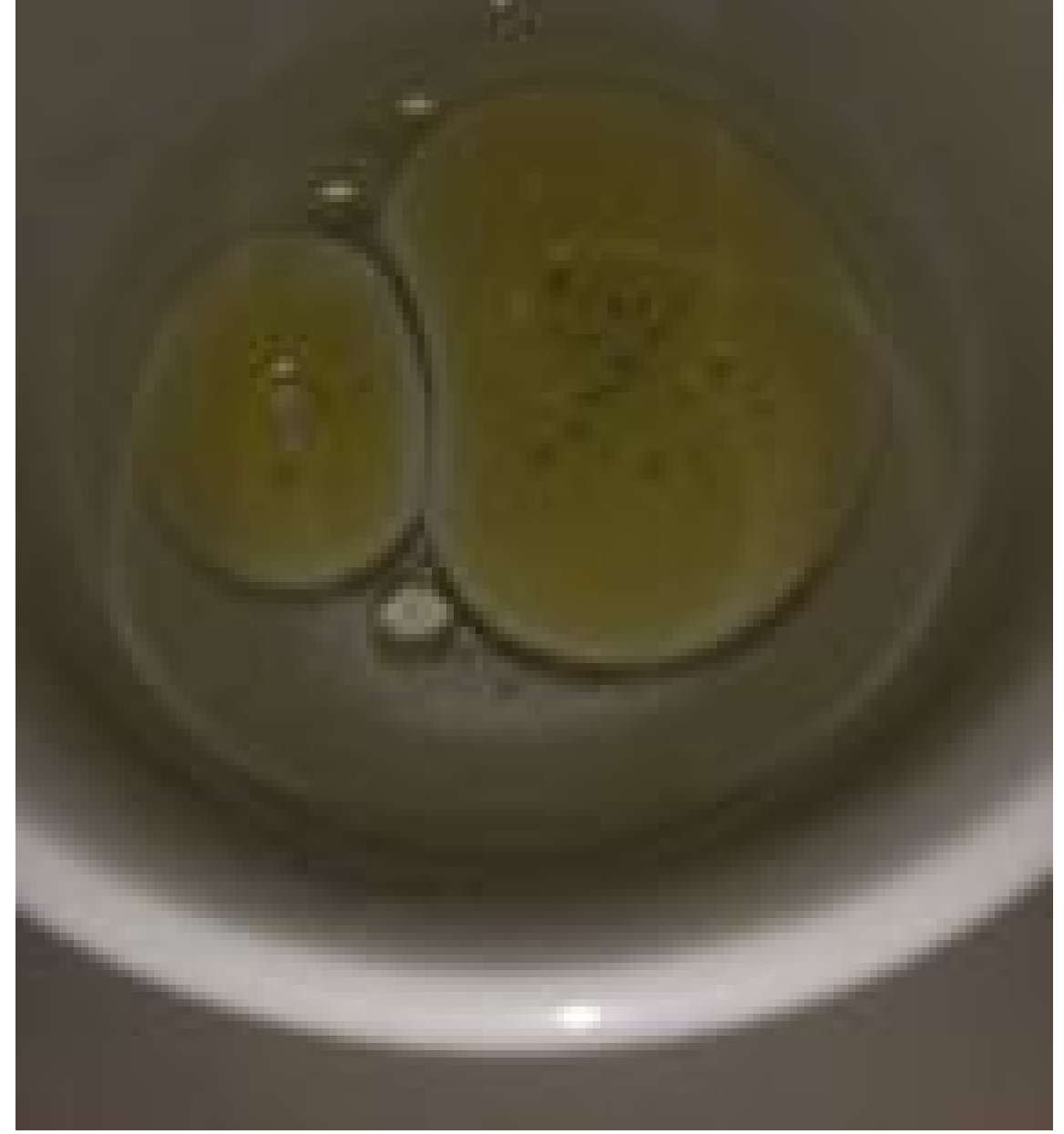

Fig. 12Fig. 13Fig. 14 


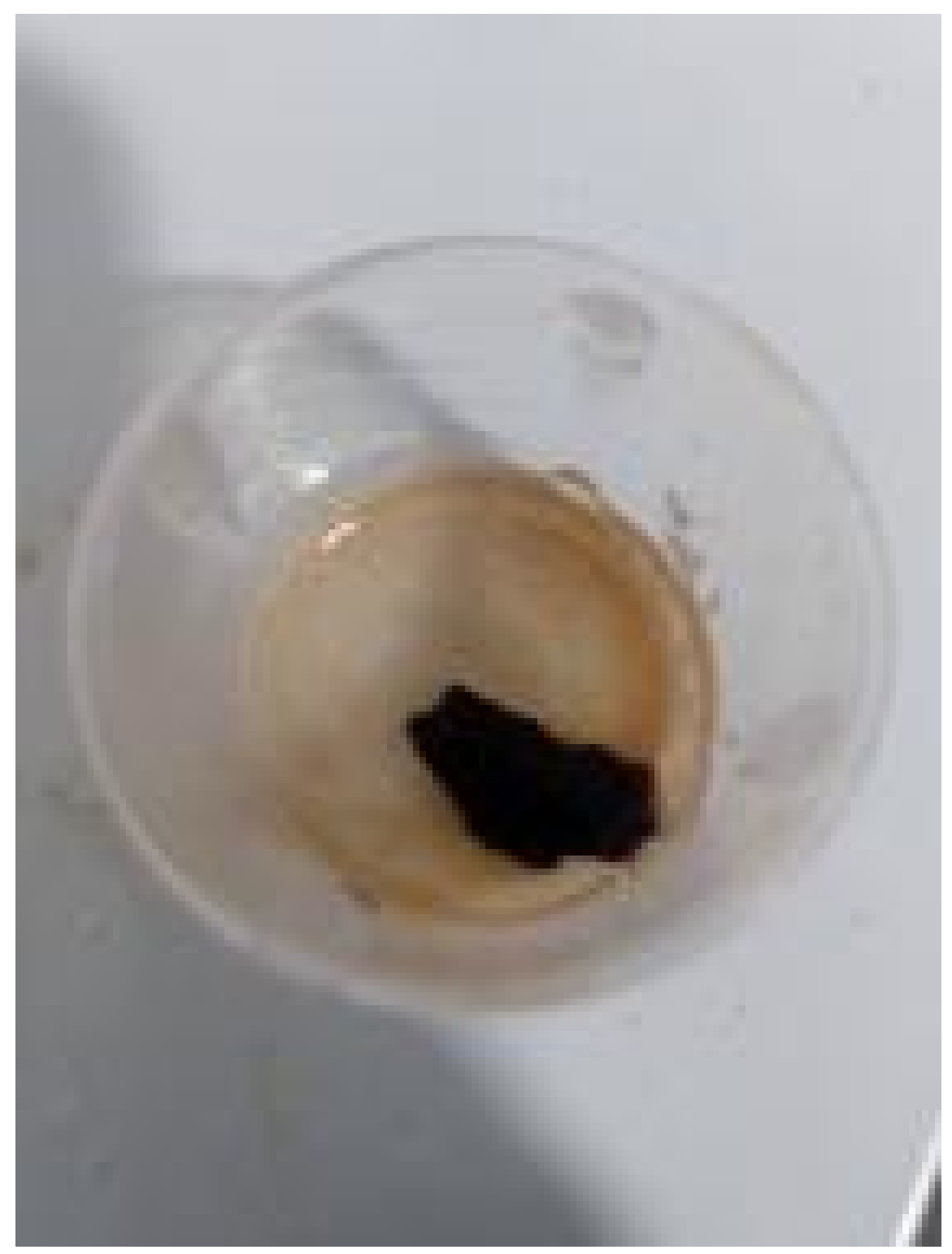




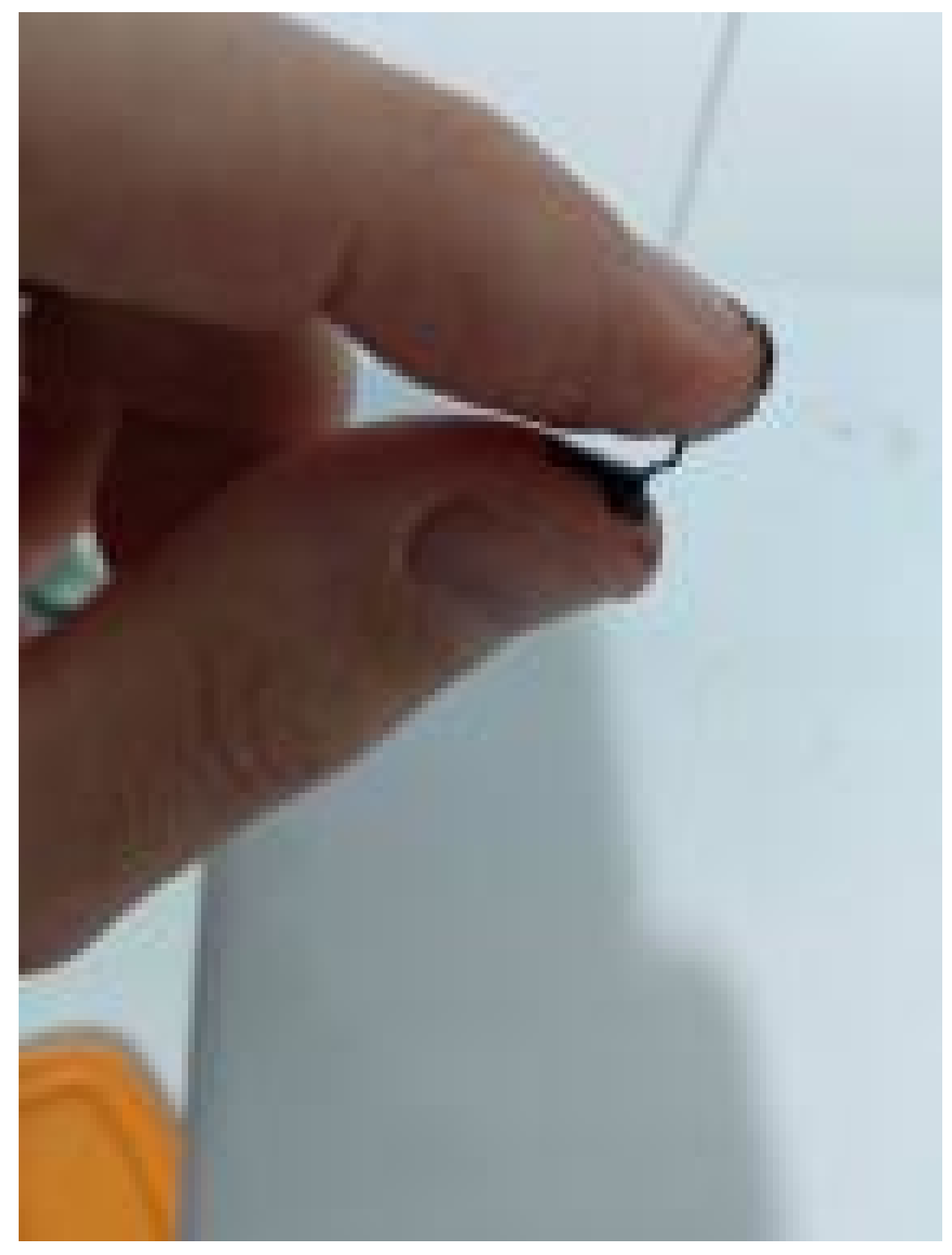




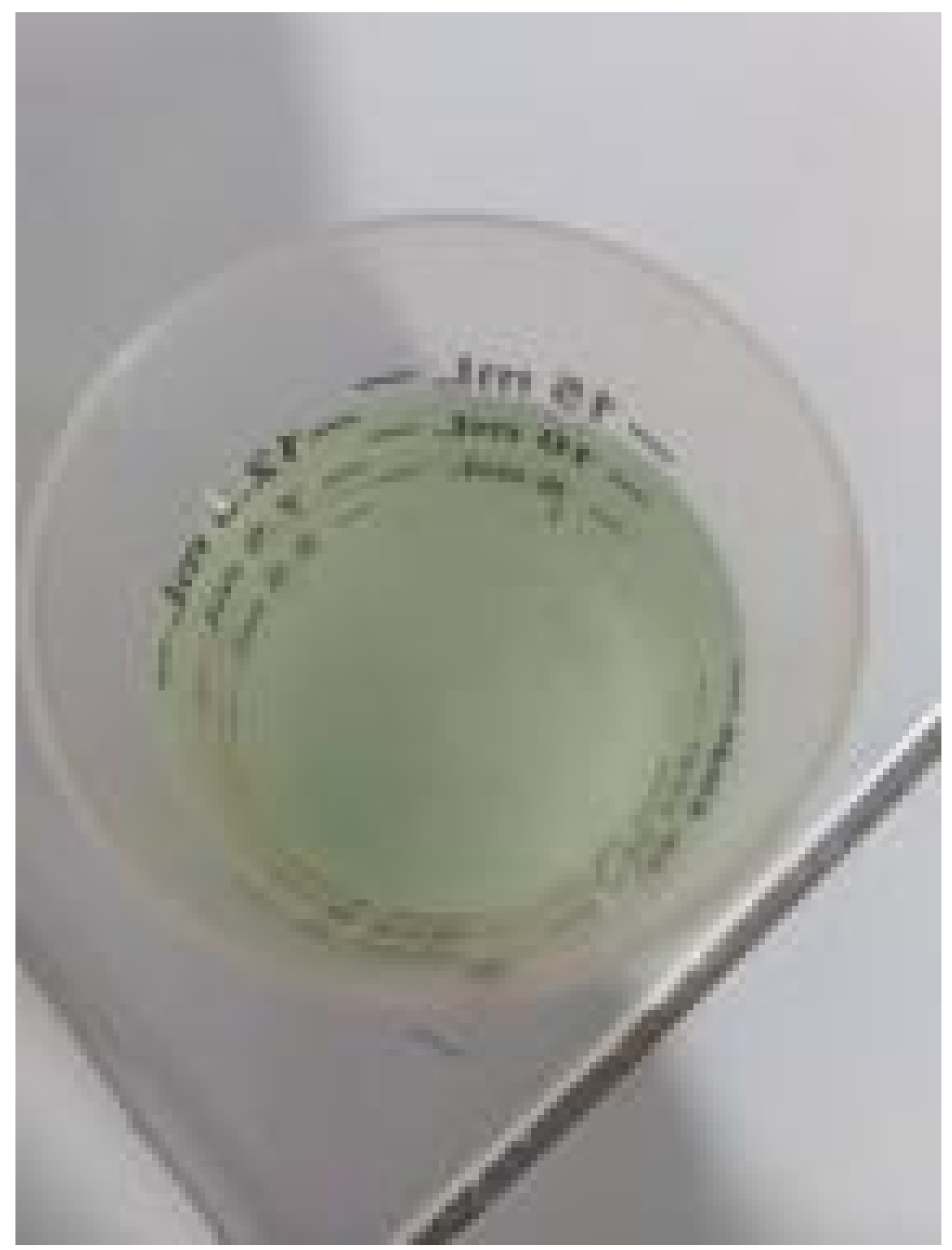

Fig. 15Fig. 16Fig. 17

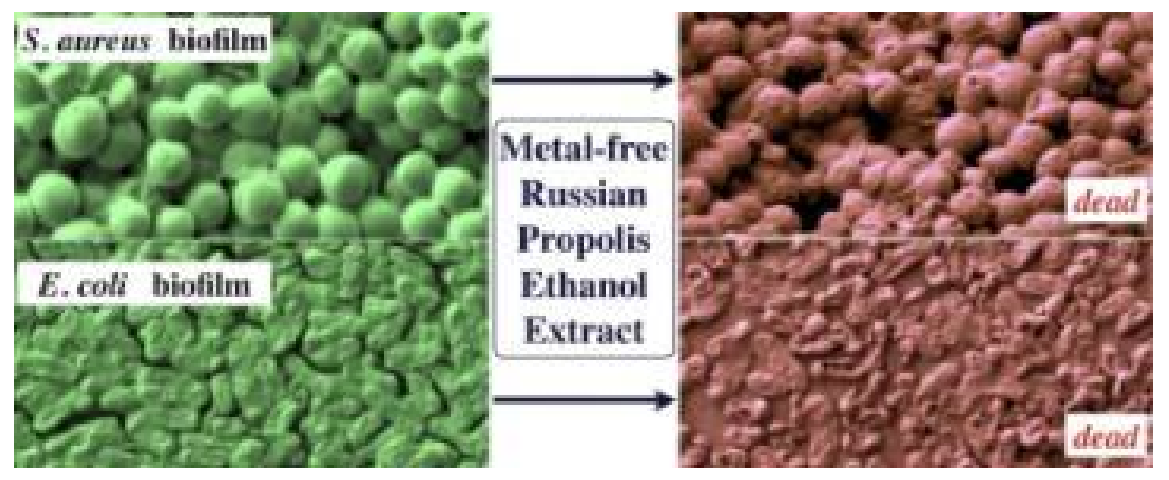


*Fig. 18 11Image used as graphical abstract in the source (quoted in references, n. 41). Retrieved from https://ars.els-cdn.com/content/image/1-s2.0-S094471131730048X-fx1_lrg.jpg. Functional quoting, comparisons with images from the present work made below in p. 40.

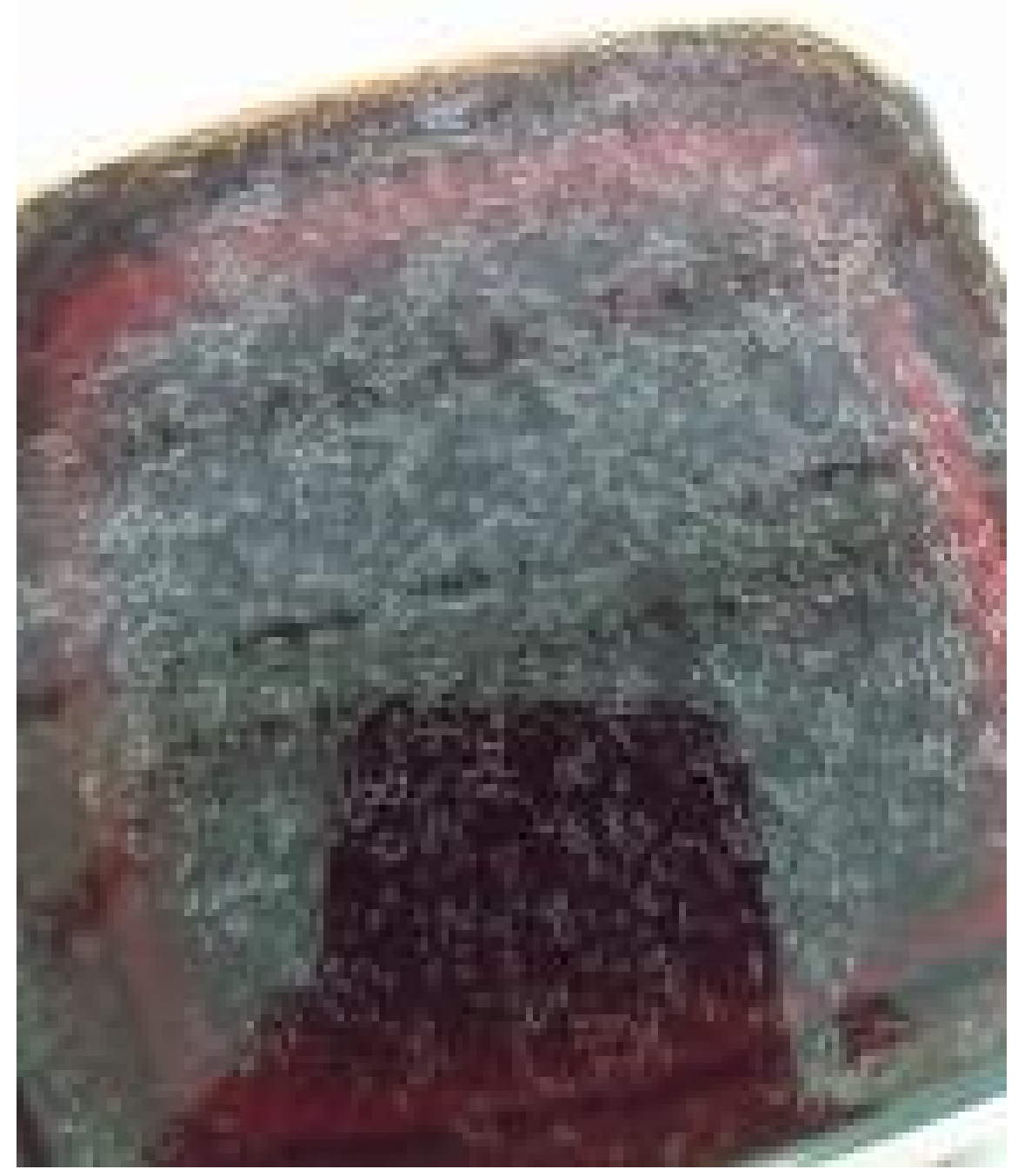




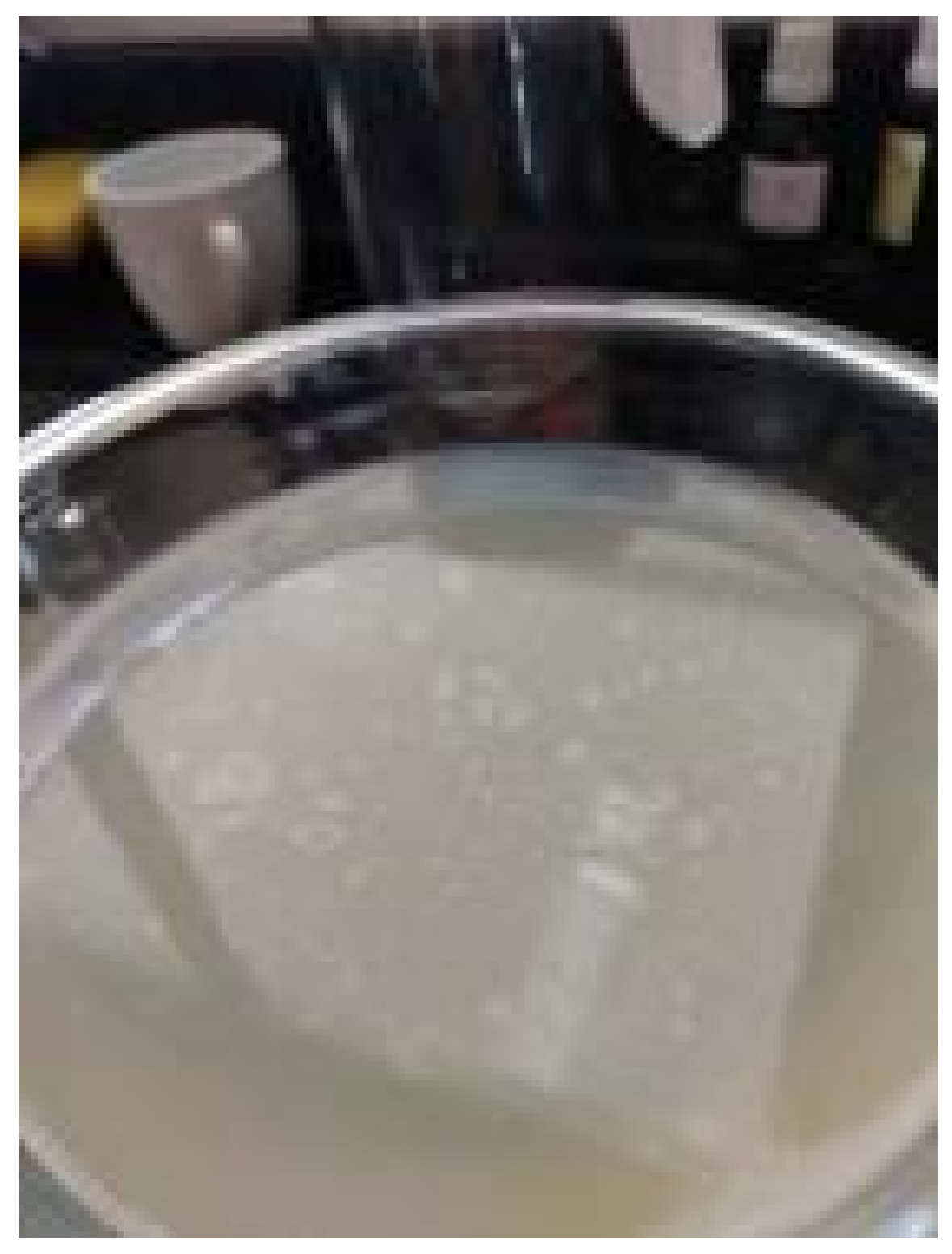

Fig. 19Fig. 20

As can be seen through the behavior/character/effects presented in the experiments, propolis demonstrated cationic behavior, the existence of significant hydrophobic parts/residues with a consequent amphipathic/surfactant character and alsohydrophilic/water-soluble parts (the tests with PAE itself demonstrated the existence of active water-soluble compounds ;PAE presented even stronger effects than PEE ; and a precipitated matter was also obtained using PAE; the matter was easily diluted again, as will be described below).

The character/effects presented were also undoubtedly strong, even with a relatively low concentration of propolis in the tested solutions $(11 \% \mathrm{w} / \mathrm{v})$. Propolis showed, when compared to the anionic detergent, a different mechanism of action (e.g., did not form foam, but was really active; did not displace the fat to the corners; and others ). Propolis also broke the surface tension of the water in a very similar manner (pushed the oregano leaves to the corners) to dishwashing detergent 
(a strong surfactant). This demonstrated the existence of significant hydrophobic parts/residues in propolis, promoting immediate action - which is quite important because in the emulsification experiments the oils could have been diluted by chemical processes and would not confirm the physical effects of the substance. In all experiments, PAE effects seemed slightly stronger than those of PEE (even with the control brands) suggesting that the alcohol may disturb/denature the active parts of propolis .

When PAE or PEE was mixed with water and oil, it seemed thatpropolis was "boiling" the oil surface , not only trapping the fat like the anionic detergent, but generating a strong physical/electrostatic effect on it. So, a very strong effect of propolis was observed on fats (hydrophobic parts) . Even coconut oil (saturated fat) was emulsified with the substance (although a larger amount of propolis was needed in comparison to the one utilized to emulsify olive oil), showing a slightly different result than the one obtained with olive oil - resembling (Fig. 19) the S. aureus biofilm (top of Fig. 18) ; while olive oil (Fig. 20) resembled E. Coli biofilm (bottom of Fig. 18) .Propolis, differently from the anionic detergent (brittle), was able to make creamy ice cream only using water and oil as additional ingredients. When used to wash hands, PAE, even without foam, seemed more powerful and effective in removing the fat than the anionic detergent. The PEE presented weaker effects on hand washing, maybe because of the alcohol and the presence of more waxy contents on it, which may have hindered the action. Thus, the existence of significant hydrophobic parts in propolis with a consequent amphipathic character of the substance was strongly evidenced by the breaking of the surface tension of water, oil emulsification and hand washing experiments.

In the Observation of cationic or anionic behavior, the cationic behavior presented by propolis generated a strong physical/electrostatic force that displaced the oil and concentrated it in the center of the cup, showing a strong capacity of the substance to be attracted and involve a huge mass of fat . Thiscationic profile of propolis was firmly demonstrated by thetotally opposite behavior presented by the substance during the experiment in comparison with the anionic detergent (Fig. 12, 13 and 14). The anionic detergent rapidly repelled the oil and displaced it to the corners of the cup . It is worth mentioning as well the result of the additional test done: propolis was mixed with the anionic detergent in equal amounts and then, drops of the mixture were added to a cup with water and oil in the surface; the effects produced were different than the ones observed when only using propolis or only using anionic detergent; it seemed that the specific effects of the substances were disturbed and the displacement of the oil did not occur; the oil remained in an intermediate state and was neither totally displaced to the corners nor to the center (showing an incompatibility possibly due to the opposite ionic behavior of the substances; no pictures were taken).

Besides this, the precipitation of PAE over the precipitated salt in the bottom of the saline solution strongly demonstrated that propolis has a large amount of cationic parts that bind to the anionic parts of the salt and precipitate (Fig. 15). Most of the PAE added to the saline solution precipitated in the bottom and formed a "resinous" compound (Fig. 16). Despite the simplicity of the experiment, given the cationic behavior already observed in the experiment described above, there is no doubt that a large electrostatic interaction occurred, and it isderived from the cationic profile of some compounds in propolis that are promptly attracted and bind to the anionic parts of the salt, also illustrating the strong electrostatic mean of action of the compounds from propolis on anionic elements/parts . PEE did not fully precipitate, maybe, again, given its waxy particles and the alcohol. After the extraction, the precipitated PAE was easily dissolved in water (through stirring; not waxy), forming a homogeneous solution (besides the obvious hydrophilic parts in the precipitated PAE; it is speculated that the extensive binding of the compounds from propolis to the single anionic elements of the salt was broken through stirring and also by the lowering of the concentration of the substances with the addition of water; but this should be analysed more carefully and with the help of more specific instruments in order to measure the power of these bindings and the possible interactions of propolis compounds with salts). The amphipathic/surfactant effects of the produced homogeneous solution on water surface were tested and remained the same (hydrophobic parts), showing again that the physical effects of the PAE are due to cationic water-soluble compounds largely present in 
propolis (in this case, pictures of the surface tension breaking experiment were not taken). Thereby, the easy water-solubility of the cationic and active precipitated PAE and hence the indubitable existence of hydrophilic parts in it (and in the PAE itself), together with the significant hydrophobic parts/residues and cationic behavior and effects of the PAE/PEE observed in the other experiments, definitely demonstrated the existence of hydrophobic residues and the cationic amphipathic/surfactant/emulsifying character of propolis, and also the watersolubility of its active compounds.

Complementing the experiments, some tests with $\mathrm{pH}$ were performed andit seemed that a slightly acidic $\mathrm{pH}$ is the ideal $\mathrm{pH}$ for the best efficacy of propolis (only tests of breaking of the surface tension of water were developed). A highly basic $\mathrm{pH}$ did not appear to disturb propolis surfactant effects too much. On the other hand, a highly acidic pH showed a strong diminution of the surfactant effects of propolis extracts, leaving them with a weak effect, but still showing some action (also tested for $\mathrm{pH}$, the precipitated PAE produced solution showed similar results in all tests).

For the sake of caution, it is worth to consider that the amphipathic behavior and effects found in propolis could also be attributed to some phenolic lipids - which propolis may contain in small amounts . However, considering that there are few phenolic lipids in propolis; they are not cationic and, as lipids, not soluble in water ; and they are not able to strongly bind to fat/membranes (only disturbing them a little) ; phenolic lipids were discharged as potential cause for the effects observed in the experiments . Another non-ionic amphipathic/surface-active known compounds, which are present in propolis in low amounts and could be the responsible for the effects observed, are the saponins. Although plausible, as no foam was observed when using propolis extracts - foam is the main effect of saponins -, and due to the strong cationic effects observed in the experiments developed,

possible effects of saponins were also discharged.

\subsection{Conclusion}

Given the findings quoted confirming the presence of all common features of AMPs in propolis (cationic charge, significant hydrophobic residues and an ensuing amphipathic character, and water-solubility); ifall these strong electrostatic character/behavior/effects can be seen with the naked eye, they must be, in a microscopic environment or in a viral size scale, really catastrophic on microbes/diseases - the term "catastrophic" was used in the American study quoted above when describing the effects of propolis ethanolic extract on resistant bacteria, and it is worth stressing that the study used a concentration of only $\mathbf{5} \% \mathbf{w} / \mathbf{v}$ in the propolis extract applied. This also leads to think about what would be the effect of an extract obtained with the focus only on the cationic peptides (possibly not denaturing or discharging them), and with a higher concentration .

As shown through these highly illustrative experiments, the evidence seems extraordinary and the effects may be more than catastrophic; they seem to be brutal, especially if propolis extract isapplied directly to the target. In this way, the results of the experiments securely support the proposed hypothesis and the experiments can be easily reproduced, even in a domestic setting , enhancing the credibility of the possible extraordinary evidence. Thus, the experimental evidence, together with the demonstrated existence of a large amount of amino acids in propolis and the common features of AMPs, completes the proposed syllogism and allows concluding that the large amount of amino acids in propolis are indeed the main parts of cationic antimicrobial peptides (AMPs) , i.e., propolis has a large amount of cationic AMPs. This is supported as well by all the existing evidence and clues in the scientific literature exposed above - such as the therapeutic effects of propolis already demonstrated so far ; bees' immune gene expression and preference for protein-rich foods; AMPs collection, synthesis and use in bee products; and others.

To sum all up, the clues and evidence exposed throughout the study, including the mechanism of action of cationic AMPs (electrostatic); the behavior/character and all the strong effects of propolis in the experiments described above, which are indubitably all features of AMPs; the effects produced by the 
PAE itself and the easy water-solubility observed with the cationic and active precipitated PAE water-solubility is also a remarkable feature of AMPs -; the cationic behavior of the propolis extracts used; the strong surfactant character/effects of the homogeneous solution produced with the cationic precipitated PAE ("resinous" compound), showing that the physical effects of propolis are due to the cationic water-soluble compounds ; the fact that, as exposed above, protein "resins" are almost $50 \%$ of propolis (amino acids, with indicators that they are part of the "resinous" antimicrobial compounds synthesized/secreted by bees and extracted from plants); the pointed fact that the other half of the compounds in propolis (which do not present the AMPs features observed, such as wax, oils, pollen and phenolic compounds ) arenot water-soluble and hence cannot be the precipitated and then dissolved compounds that showed surfactant effects (besides the strong effects showed by the PAE itself, securely already demonstrating the existence of water-soluble active ingredients in it); and the evidence in the study cited of the existence in propolis of a large amount of the amino acids proline and arginine, which are usually main components of cationic AMPs - bee AMPs areall cationic and tend to be arginine and proline-rich peptides ; all firmly demonstrates that the strong cationic amphipathic/surfactant/emulsifying character/effects observed through the experiments with propolis can only stem from this large amount of amino acid derived bioactive cationic compounds (AMPs) that exist in the substance .

In addition, it is undeniable that, given the brutal effects observed with the naked eye in the experiments and the medicinal properties of the substance already demonstrated by numerous studies, the therapeutic mechanism of action of propolis is physical/structural and directly derived from the effects produced by the cationic AMPs existing in it . The mechanism surely works through the powerful electrostatic force exerted by the cationic peptides on the anionic moieties of the organism/agent with which the substance interacts (this, as seen, produces a series of phenomena in which one leads to the next, like a cascade). This mechanism of action of propolis, beyond the attraction and involvement of a huge mass of fat seen in the experiment of observation of the cationic behavior of propolis, was remarkably observed through the prompt and strong electrostatic binding of the cationic compounds from propolis to the anionic elements/parts of the salt in the experiment of precipitation on salt.

Besides all the reasons previously quoted, the inference that the therapeutic mechanism of action of propolis is physical/electrostatic and derived from the AMPs in it is directly corroborated by the fact that AMPs work mostly through electrostatic interactions andthe demonstration, in the American study already discussed above, of the physical/structural mechanism of action of propolis against resistant bacteria - similar to lytic peptides/cationic AMPs, binding specifically to the cell membrane of bacteria and inducing death through catastrophic cell lysis . Furthermore, the physical action of propolis is also reinforced, through association, by the fact that, as already quoted, propolis has a huge amount of arginine and proline - usually main components of cationic AMPs -, and bee AMPs are all cationic and tend to be arginine and proline-rich peptides, which are known to have a mechanism of action with a broad spectrum of antimicrobial activity and can kill bacteria, fungi, viruses and protozoan parasites. Lastly, this physical/electrostatic mechanism of action of propolis is also shown, more indirectly, by the finding that theoverall antibacterial activity of honey is due to thecell wall lytic bactericidal activity of bee MRJP1glycoproteins, demonstrating, in this way, in a bee product, AMPs that have the same physical/structural/lytic mechanism of action observed in the American study with propolis .

Therefore, beyond the initial claim - it was possible to observe the power of the effects as well -, it cannot be rejected that propolis has a large amount of cationic AMPs, which produce strong effects ; i.e., propolis has, given the significant hydrophobic residues of the water-soluble cationic peptides that abound in it,strong amphipathic/surfactant character and its therapeutic mechanism of action is physical/structural, through electrostatic force; thus, in order to produce effects, the cationic peptides in propolis bind, by attraction, to anionic moieties of the organism/agent with which the substance interacts, and generate a cascade of phenomena. 


\subsection{Discussion}

As an initial observation that must open the discussion of the experiments, the studies developed with propolis until now will be generally commented. Despite the possible mistaken focus quoted above and the few comprehensive in vivo studies in humans, propolis is still sparsely demonstrating antimicrobial and disease fighting effects (mainly immunomodulatory and cytotoxic). Maybe all the research done until now, even the ones that used propolis as a whole, not isolating one or some compounds, were also not done in the desirable way. Given the pointed existence of a large amount of cationic AMPs in propolis, even considering that some of the peptides may be truly resistant, most of them possibly can be easily denatured. Hence it is believed that propolis extraction methods through acids, alcohols, heat and other physical stressors all have the potential do impair the efficacy of the final product and the focus only on its phenolic compounds is surely impoverishing the extraction. Besides this, most experiments in vitro use acid in the solutions and the lab cell cultures, viruses and bacteria may not reflect the real disease environment - with the correct $\mathrm{pH}$, loose proteins/receptors, electric charges and other signals/conditions to the most effective action of propolis "inside an organism" -, which enables its electrostatic effects.

Moreover, really important factors that seem to matter in the final result of the experiments are possibly not being taking into consideration, such as: the application directly to the infection/disease site; dosage; obtaining method; concentration of the extract and period of use of it. The administration method seems to be also extremely important and studies in vivo vastly use peroral administration. It is believed that, as already said, despite few studies in the area and only focusing on phenolic compounds, given the high affinity for bacterial membrane and products, propolis peptides may heavily bind to gut bacteria, hindering the ample absorption and harming the gut flora, especially if administered in great amounts.

One other possible problem pointed out in various studies is the fact that propolis from different regions may have different effects. However, it is believed that this difference in the effects may be due to problems in the extraction, application far from the disease site, formulation of the experiment and others. Considering that the cationic AMPs existing in propolis are, as discussed, genetically pre-programmed, with few differences among bee species and places, and they have physical mechanisms of action, they are supposed to work anywhere - the way to test it is very simple as the experiments described above showed.In summary, propolis possible main active ingredients work through physical contact (electrostatic action), so, it is believed that the lesser the previous interaction with organisms/agents that the cationic AMPs may bind or be denatured by; the higher will be the power of the substance to fight infections/diseases. In this way, it is believed that, from now on, researchers should have all these considerations in mind in order to extract and measure all the benefits of this extraordinary substance.

As one more observation, an interesting finding, which may show the strong power of AMPs from propolis and is worth mentioning before other discussions, is the fact that propolis is also used by bees to mummify intruders in the hive. Perhaps the intricate mechanism of action of the mummification process in Egypt - they commonly used propolis as an embalming agent - was obtained through the use of propolis, which have already been found, together with honey, in Egyptian mummies . It is also known that honey may last forever and, as quoted, the antibacterial mechanism of action may be the same of propolis, working through antimicrobial peptides (AMPs). The half-life for the hydrolysis of a typical peptide at neutral $\mathrm{pH}$ is estimated to be between 10 and1000 years, thus, maybe the peptides (AMPs) in propolis (and in honey) are so special that, with all the phenolic compounds and other "preservers" in the substance, they may last forever .

These peptides are so special that the possible extraordinary existence of a large amount of cationic AMPs in propolis, if definitely confirmed, may open an entirely new and promising world for science. As shown, propolis possibly has demonstrated strong antimicrobial and disease fighting properties, mainly if applied directly to the infection/disease site in the necessary amount, and this should be extensively explored by science as a potential extremely powerful therapeutic agent. In a simple manner, propolis seems to be basically a more complex and selective/non-toxic "detergent/soap" that can be safely used internally and consumed by humans with no side effects (especially if compared to regular 
detergents). As quoted above, analogies between membrane-active peptide and detergents have been studied ; however,AMPs, as seen, besides amphiphilic detergent/soap-like properties, may be more powerful on viruses and other microbes than any existent detergent. AMPs have especial properties, not just involving the microbes, but producing a broad range of other effects on the disease/infection site - even on host's cells. Also, as already mentioned, AMPs have another extraordinary feature that is low cytotoxicity to host health cells, so, the mechanism of action of propolis may be selective. This happens with AMPs, as discussed above, due to the fact that the health cell membranes possess a higher percentage of cholesterol and that the cationic property of AMPs mainly contributes to cell selectivity, because the surface of bacterial membranes - and sugar chains of virus glycoproteins- are more negatively charged than that of mammalian cells. Also, as will be seen, sick cells and disease sites are more negatively charged than healthy cells/sites. The dose dependence of propolis also may be explained by its amphipathic, detergent-like nature, because detergents have a limited solubility in the aqueous phase, characterized by the $\mathrm{CMC}$, or critical micelle/monomer concentration;above the CMC, detergents form micelles. In the presence of membranes, detergents can also partition into the membrane andsufficient detergent will lead to transfer of membrane components (even biological membranes), including membrane lipids and membrane proteins, into the detergent micelles . As already quoted about AMPs above, after raising the peptide/lipid ratio, pores begin to form in the critically thin membrane, and the peptides orient themselves perpendicularly and insert into the bilayer ; so, it is a very similar mechanism.

Besides the detergent-like effects, it is worth mentioning that thepositive-negative, simple "yin and yang" mechanism described in AMPs and associated with immunity/infections/diseases seems to be a really promising subject to be explored by science and surely deserves more attention from researchers - Despite the enormous amount of studies and the focus mostly on the complex chemical compounds involved, perhaps the code is simpler and only dual (positive versus negative). Maybe it is already cracked and physics, with a less complicated approach, brought a better solution for disease fighting than the other science branches have been trying to do for a long time. It is a possible simple dual, physical and electrostatic mechanism that seems to rule most diseases. That is, in a simple metaphor, when the organism is sick, or an intruder is trying to or has invaded it, or a cell rebels (the intruders/rebels are also always out of the pattern and leave pieces, clues) they sound a loud alarm, especially for the first defense army (human body innate immunity and its AMPs). This army is tailored to keep searching enemies electrostatically, instantly binding to them or to substances that they depend on and others, and neutralize their actions, preventing the invaders or the rebel cells to reproduce in huge amounts and domain the entire organism. The AMPs used by the immune system have a broad spectrum of different function soldiers, there are soldiers that can attack the enemy armor directly, others can cut the enemy supplies or prevent them to get to the cells by blocking the road, they even can get into host's cells/rebel cells "mind" and reprogram it to "commit suicide" or become a friend and help.

It is as simple as it looks, the first and most important lines of defense, which are the AMPs, are strongly positively charged and keep the "electrostatic patrol" against the negatively charged offenders in a high-tech and natural selective way, perfectly distinguishing the invaders from health cells. However, sometimes, given a series of reasons, mainly because the body defense system is generally compromised by "self-sabotage" and/or "biological weapons" that confuses the communication and the whole system, the organism "electrostatic patrol" is diminished and incapable to fight against the enemies. Thus, maybe now the possibility of "implanting" a powerful cationic electrostatic army in any amount needed to fight diseases/infectionswith ample success - engineered and perfected by bees for 120 million years, and that can, given the similar mechanism of action, perfectly communicate and work in synergy with host's defenses - is closer than ever imagined .

In order to illustrate this dual mechanism, as the cationic nature of AMPs was already extensively discussed, the negative charges of infections/diseases can be shown. Also, to spare time, as already seen, bacteria, viruses and some moieties from both are highly negatively charged and AMPs are an effective tool against them, so, this will not be extensively exposed again. Regarding viruses, just an interesting observation must 
be stressed, in 2010, a leader scientist in the growing field of physical virology, which studies the physics of viruses, used the following metaphor when talking about antiviral drugs in an interview: "the overall problem with these medications is that they are like a football defensive squad trying to tackle individual players on the virus's team, only to discoverthe virus has substituted players that can break through their defenses " (...) and continued, saying that he hopes to develop methods that in effect would keep the virus's team out of the stadium altogether. The DNA inside a virus has a distinct negative electrical charge, he said, so one way to thwart the virus might be to find small positively charged molecules to counteract that, "If we can find a small peptide with a positive charge and this can interact with the negative charge, it might either keep the DNA inside or decrease the strength of its release". So,maybe physics can really outsmart viruses and the possibility that is under scrutiny in the present study, i.e., implanting external cationic AMPs from propolis, seems to be the most promising way to do this . Even in human immunodeficiency virus treatment, natural AMPs seem to be promising anti-HIV candidates .

Inside this context, a devastating disease that perfectly exemplifies this dual mechanism and must be discussed is cancer. Many studies have demonstrated that cancer cells are more sensitive to AMPs than normal cells, because the cytoskeleton of cancer cells is not well developed in comparison with that of normal cells(the high metabolism in cancer cells causes the potential changes in membrane, cytoskeleton or extracellular matrix of cancer cells).Cationic AMPs associate with the high acidic phospholipids on the outer surfaces of these cancer cells . These peptides easily insert into the lipid membranes and form ion channels or pores to eventually destroy the cancer cells or result in leakage of cell contents . A recent (2019) and extremely interesting study about detection of cancer cells based on surface electrical charges suggested that all tested cancer cells(22 cancer cells of different organs) are negatively-charged and normal cells are either charge-neutral or slightly positive . For diagnosis, cancer cells can be detected, electrostatically bound, and magnetically separated in blood by charged and super-paramagnetic nanoprobes; and, in therapeutics, circulating cancer cells (CTCs) can be filtered and removed in a continuous fashion to reduce the risk of cancer metastasis . If successful, this new nanotechnology will revolutionize early cancer diagnosis and potentially enable new therapeutics in clinical settings .

Perhaps this kind of expensive desired technology quoted is being already developed in nature for free, and the "technology" from bee cationic AMPs may be the future of cancer fighting . It is possible that the COVID-19 outbreak brought to light a highly effective therapeutic method against a broad range of diseases. The selective mechanism of AMPs and the possibility of implanting them in the disease site in any amount needed, working in synergy with the host immune system, may revolutionize cancer treatment, becoming a cheap and non-toxic option. This is also a possible indicator for humans of the need to respect and preserve nature and also to take better care of their health, avoiding that the electrostatic mechanism of defense gets compromised - e.g., in cancer, in which the immune system of the diseased organism is maybe weak and gets deceived, becoming incapable to recognize or fight and prevent cancer cells growth, possibly due to "self-sabotage" and/or external "biochemical weapons".

Despite the focus on the antioxidant components of propolis, various studies quoted in the present work stated that propolis may have anti-cancer effects . However, to really illustrate the line of thought exposed and clarify the arguments that cationic AMPs from propolis may be a real powerful tool to fight diseases and even cancer, it can be quoted an interesting review about the anticancer activities of bee products. The study found that peptides in bee products - specially melittin, the major protein component in bee venom, a $\omega \alpha \tau \varepsilon \rho \sigma o \lambda \cup \beta \lambda \varepsilon, \varsigma \alpha \tau \iota \circ \vee \iota s, \alpha \mu \pi \eta \iota \pi \eta \iota \lambda \iota \varsigma \alpha-\eta \varepsilon \lambda \iota \varsigma \alpha \lambda \pi \varepsilon \pi \tau \iota \delta \varepsilon$-induce apoptotic cell death in vitro in several transformed (cancer) human cell lines, including those derived from renal, lung, liver, prostate, bladder and lymphoid cancers .Bee venom also inhibits the proliferation of carcinoma cells and tumor growth in vivo due to the stimulation of the local cellular immune responses in lymph nodes, so, these bioactive natural products may, therefore, prove to be useful as part of a novel targeted therapy for some types of cancer, such as prostate and breast cancer. Thus, this is basically only one AMP - maybe all the others together like in propolis 
may be much more powerful - and it has direct and indirect effects, "waking up" the host immune responses. "Imagine a new cancer treatment, based on the cruise missiles principle . A submicroscopic rocket with an automatic search head is launched in the body through injection. It searches out cancer cells and destroys them without attacking normal healthy tissue. Such a wonder weapon does not exist yet, but there are indications that it could be available in the near future". These words were published in 1981, in the Wall Street Journal. Perhaps this mechanism envisioned forty years ago (still extremely expensive to be developed and not viable) has always existed in nature and bees may give it for free in abundance .

Still illustrating this extraordinary electrostatic mechanism and the loud alarm sounded by diseases, a recent study showed how the body may initially respond to infections, concluding that an increase in the internal negative charge of the cell triggers a signaling cascade activating an innate immune response capable of controlling infection. This increase in the internal anionic charge of cells is likely to activate several cytosolic proteins via the interaction of polycationic motifs with these negative membrane domains, a prerequisite condition necessaryfor the transcriptional activation of several genes that modulate macrophage functions and trigger an innate immune response capable of controlling infection rapidly and efficiently . Thus, the study stated that macrophage reprogramming by negatively charged membrane phospholipids controls infection . So, it can be suggested that cationic motifs from innate immune system, attracted by the anionic charges in diseased cells, are alsopossible "physical/chemical messengers" that give the signal to cells start reacting and how to react - "messages" seem also with AMPs and indirectly observed through propolis immunomodulatory effects already demonstrated by science. In this line, as cited above and seen in the case of cancer, it is worth stressing again thatcationic antimicrobial peptides can also act in synergy with host molecules, such as other cationic peptides and proteins, lysozyme, and also conventional antibiotics, to kill microbes ; it has been found that certain peptides are produced in large quantities at sites of infection/inflammation, and their expression can be induced by bacterial products such as endotoxic lipopolysaccharide (LPS) and pro-inflammatory cytokines , such as tumor necrosis factor-alpha (TNF-alpha) .

Besides the electrostatic mechanism, one more sign that sounds the body alarm is the $\mathbf{p H}$ alteration in the infection/disease site. Thedevelopment of an acidic tissue environment is a hallmark of a variety of inflammatory processes and solid tumors. In fact,values of $\mathrm{pH}$ ranging from 5.7 to 7.0 are usually found in a number of solid tumors such as breast cancer, brain tumors, sarcomas, malignant melanoma, squamous cell carcinomas, and adenocarcinomas. Both the innate and adaptive arms of the immune response appear to be finely regulated by extracellular acidosis in the range of $\mathrm{pH}$ values found at inflammatory sites and tumors. These findings seem to be in accordance with AMPs means of action, as a group of AMPs that are increasingly being reported are those that utilize pH dependent antimicrobial mechanisms, and are primarily cationic, although a number of anionic examples are known. Some of these molecules exhibit high $\mathrm{pH}$ optima for their antimicrobial activity but in most cases, these AMPs show activity against microbes that present low pH optima , which reflects the acidic $\mathbf{p H}$ generally found at their sites of action. With propolis, for example, the pH of it is slightly acidic, with values close to 5.0. This, as seen, may mean that the other substances, mainly phenolic compounds, besides the peptides that are the possible active ingredients, are also enhancers that create a more acidic environment and enable the cationic AMPs from propolis to work better - as said before, this happens because the AMPs in propolis have to act "externally" and be "always ready", despite originally designed to act and be triggered inside an organism, using chemical signs and enhancers that occur in the disease site. So, the possible preservers/enhancers compounds in propolis may not be necessary inside a sick organism because the own organism is capable of signaling correctly by regulating the $\mathrm{pH}$ in the infection/disease site and enable AMPs to act in the optimum range .

In this possible "yin and yang" background of diseases, the cationic AMPs from propolis may be a really viable and cheap solution for the world's greatest health issues, especially in developing countries that are the 
biggest producers. Until now, science has been considering that, given the high production costs of AMPs and their low bioavailability in nature, tailoring new AMPs or strategizing approaches to increase the yield of AMPs is pertinent to fight antimicrobial resistance, a critical public health threat. At least700,000 people die each year due to drug-resistant diseases. Also, acute lower respiratory tract infections have beenamong the top three causes of death and disability in both children and adults, and it is estimated that they cause nearly 4 million deaths annually. Perhaps thetreatment of respiratory tract diseases is the first and most promising alternative that propolis may provide, and it is ready to be applied right now through inhalation - as will be discussed below, especially regarding COVID-19.

It is worth mentioning again that the mechanism of propolis cationic AMPs, given the electrostatic/physical action, is designed to not be resisted by any pathogen . In bacteria, the most complex and resistant pathogens, their resistant nature stems from the chemical rather than the physical means of inactivation of antibiotics. Bacteria are incredibly adaptable and over time they develop defenses to the chemicals used in antibiotics, but "they have no way of dealing with a physical attack ", as said by a group of scientists, already mentioned above, trying to develop and use nano-sized particles of magnetic liquid metal to shred bacteria and bacterial biofilm. It is never enough to remember as well that possibly all propolis types, irrespective of the origin, are supposed to have cationic AMPs and produce infection/disease fighting effects - perhaps in the future, after cataloging them, different propolis AMPs from various locations may be mixed in one product to act in even bigger synergy. Again,propolis possible main active ingredients work through physical contact (electrostatic action), so, it is believed that the lesser the previous interaction with organisms/agents that the cationic AMPs maybind or be denatured by; the higher will be the power of the substance to fight infections/diseases. Thus, it may be extremely necessary to apply the AMPs from propolis as a whole to enable their synergistic effects (never isolating; as said, despite the same electrostatic cationic mechanism of action, each peptide, given the structural/physical form, may have specific effects), and apply them directly or close to the infection/disease site, mainly through parenteral administration, in the correct dosage, and for the necessary period . The AMPs from propolis are not supposed to act in a general chemical function; possibly they are selective and have highly specific targets (negatively charged parts of the organism/agent)that should be directly sought - maybe the groups of scientists mentioned in the American study cited above as those who believe that the mechanism of action of propolis is very specific on the targets are, in a way, correct.

In regards to direct application, despite the possibility of inhalation that will be discussed below, in some infections/diseases, it may be the difficult to directly achieve the infection/disease site, but science is totally able of researching the best way and doing it effectively in a short time. Besides physics, it is also a matter of math ,the necessary concentration, dosage and time should be found . As seen above, even though AMPs having other active mechanisms, the critical micelle concentration (CMC) of the "detergent" must be achieved and the peptide/lipid ratio must be raised to damage microbe's membranes and other parts. In some cases, the way that bees use propolis, making an envelope to protect the hive , should be imitated to make propolis effective. These are simple considerations but may have prevented propolis full action on human infections/diseases since it was discovered . A study, for example, which administered $150 \mathrm{mg} / \mathrm{kg}$ of water soluble derivative of propolis intravenously, intraperitoneally and orally to mice, found that theimmunomodulatory effects were strongly dependent on the route of propolis administration, with the parenteral administration seeming the best route .

Therefore, in this electrostatic defense mechanism that loudly sounds alarms in case of infections/diseases, there is no doubt that,as a state of art "technology", with $\mathbf{1 2 0}$ million years of evolution, the cationic AMPs from propolis are a powerful electrostatic weapon that may be successfully "implanted" in humans in any amount needed, also working in synergy with host's defense, against a broad range of infections/diseases. The question from now on is how to correctly produce propolis (possibly feeding bees a protein-rich diet); best extract the AMPs (not denaturing the peptides and preserving all the power); possibly concentrate and apply them intact to the disease site or as 
closest as possible (study, catalog and maybe combine different AMPs from different bee species; use them mostly through parenteral administration, and study all physical variables involved) andlet the "cruise missiles"/the "foreign army" do their job . AMPs are a "whole immune system", imitating host innate immune defenses and working in synergy with them. AMPs are supposed to bemuch more effective than any drug or technology ever invented. They are not only simple anti-inflammatories, antimicrobials or cancer/disease fighting agents that act through chemical processes. As already vastly discussed, summarizing, cationic AMPs possible synergistic physical/electrostatic/structural and not only chemical/functional mechanisms provide a broad range of direct effects on infections/diseases, and a strong immunomodulatory effect in the host as well (possible physical/chemical messengers), furthering the general homeostasis by preventing the excess inflammation and oxidation and all the consequences that stem from them. That is, propolis may be able to bring together, in the same substance, the expected effects of various therapeutic agents.

It is extremely fantastic to think that this complete immune system may be "implanted" in other organisms. Studies about propolis, even sparsely and with a different focus, have been demonstrating strong medicinal properties of the substance, which indicates that the "implant" may be totally possible. This is maybe due to the similarity of bee cationic AMPs with all innate immune systems that, despite the biochemical complexity, evolved in a really similar manner,allowing them to "connect" (even with the adaptive; AMPs are possible physical/chemical messengers as well) and work together against pathogens/diseases. As said, propolis is supposed to imitate bees' individual immune system in a stronger manner; so, it is possibly comprised of several different species of cationic AMPs (there are more than $\mathbf{3 0}$ already identified in bee genes and products, and there may be several from plants too ). Possibly,each AMP has different means of action in infections/diseases, with an enormous range and combinations of effects. Maybe in a near future they will all be identified, cataloged and studied in order to thoroughly understand this extraordinary mechanism and allow humans to fully benefit of it. Perhaps SARS-CoV-2 ended up leading to the discovery of a treatment for several diseases and will soon allow saving millions of lives and costs with public health.

Lastly, besides a possible therapeutic agent to be used now against SARS-CoV-2, which will be vastly discussed in the next sections, given the extraordinary world of possibilities in the infection/disease fighting field brought to light, which science should extensively explore, the present section will be finished with a table of the possible advances that the cationic AMPs from propolis may further bring to the health area. As already said, AMPs have been receiving a lot of attention from science in the last years given their broad spectrum of antimicrobial activity, including anti-bacteria, antifungi, anti-viruses, cancer fighting properties, and important role in immunomodulatory and inflammation responses .

Possible Effects of the Cationic AMPs of Propolis

Virus

Bacteria

Fungus

Cancers

Respiratory tract diseases (through inhalation)

Allergies

Autoimmune diseases

Implants

Wound healing, dermatitis

Anti-aging

Premature babies

Corticosteroids and other immunomodulatory drugs

Detergent

Pandemics
Direct effects on all viruses and immunomodulation in the ho May be really effective against all sorts of bacteria given the As the studies about propolis have been already demonstrati Cheap, selective, non-toxic treatment alternative for all can Cheap and strong treatment for a broad range of them given May clear the allergens as a detergent and act as a strong im As the cationic AMPs from propolis have potent immunomo As the studies quoted in the present work, propolis has been Studies have already been demonstrating that propolis is effe Given the Immunomodulatory and complex antioxidant effec As a "whole immune system" that may be "implanted", sinc As a strong immunomodulatory, with low to no side effects, As a potent antimicrobial and having low toxicity, may be us May be an effective prophylactic measure, possible provid 
Possible Effects of the Cationic AMPs of Propolis

Other diseases

These ample antimicrobial and immunomodulatory effects sh

\section{Propolis Basic Pharmacology}

Before entering in the specificities of propolis against SARS-CoV-2, as a possible treatment, it is necessary to understand its pharmacology and its possible administration through inhalation, which is going to be discussed through this and the next section.

\subsection{Composition and Mechanism of Action}

Propolis is a well-known natural resinous mixture produced by honeybees from exudates from buds, plants, poplars, conifers, birch, pine, alder, willow, palm, Baccharis dracunculifolia , and Dalbergia ecastaphyllum - Raw propolis consists of about 50\% resins, 30\% waxes, $10 \%$ essential oils, 5\% pollen, and $5 \%$ various organic compounds, including flavonoids, phenylpropanoids, terpenes, stilbenes, lignans, coumarins, and their prenylated derivatives, with $>300$ different substances identified . The latter $5 \%$ substances, as seen, are the only ones vastly studied. The precise chemical composition of propolis depends on geographical location, botanical origin, and bee species involved. Propolis of different origin contains different constituents and some of them are present in many samples from different places. Some constituents are present in sample from specific plant origin. The main chemical components in propolis, as already shown, studied mostly in terms of pharmacological activity, are pinocembrin, pinobanksin, caffeic acid phenetyl ester, artepillin C, cinnamic acid, p-coumaric acid, caffeic acid, ferulic acid, isoferulic acid, chrysin, galangin, kaempferol, and quercetin . Being the main constituents, flavonoids contribute greatly to the pharmacological activities of propolis. So, as already quoted, all the studies are focused only on the phenolic compounds

An immense variety of Propolis types have been documented and it seems that most of them can be successfully applied in rodents and humans as an anti viral agent. All propolis types, irrespective of origin and consequently the compounds they contain, have shown antimicrobial activity indicating it is rather the collective of propolis compounds rather than the individual compounds that result in its antimicrobial activity. As an example, one study that used Brazilian and Chinese propolis found no significant differences between them regarding their effects on diabetic mice . Despite having more than 300 different identified substances and the attempts of a few researchers to isolate one or some of the compounds of propolis in order to produce medicines, it seems that the substance only reaches its designed effects in nature's original form (maybe the AMPs can be separated from the phenolic compounds and others; but these peptides must stay together to be fully effective). As already mentioned, all propolis from anywhere may have cationic AMPs (antimicrobial peptides) and be able to exert antimicrobial and disease fighting effects, depending only on the correct extraction, concentration and application methods .

Besides all biochemical components quoted, propolis, as showed above, is comprised of a vast amount of free amino acids and their total concentration in propolis is over $40 \% \mathrm{w} / \mathrm{w}$, witharginine and proline constituting over $\mathbf{5 0 \%}$ of the crude acid extract . Thus, despite no studies about them, it is believed that the disease fighting effects stem from these amino acids and from the compounds derived from them rather than from the phenolic compounds. It is worth mentioning again that the pH of propolis is slightly acidic, with values close to 5.0 . Therefore, the active infection/disease fighting ingredients, as the present study has been pointing, are the cationic antimicrobial peptides (AMPs) existing in propolis, which, it is worth stressing again that, given that each one of them may have specific and complementary mechanisms, they need to work as a set, in synergy, and not be isolated . So, as AMPs were vastly studied above, they will not be fully detailed again in this section. In summary, propolis has various possibleantimicrobial/disease fighting electrostatic mechanisms of action through cationic AMPs. They are supposed to act directly on the infection/disease site, with propolis 
positively charged AMPs physically binding to all parts in the structure of the microbes/host's cells and their products/substances that are negatively charged,potentially annihilating the microbes and/or modulating host's immune system .

\subsection{Treatment Time and Dosage in Viral Infections}

As the present studied is focused in the treatment of COVID-19, this section will try to approach propolis use specifically in viral infections, but the statements can surely be applied to other cases. Also, as already discussed, all scientific references are focused only in the phenolic compounds of propolis.

An article stated that the difficulty with prescribing an accurate dose of propolis based on the investigated population, dosing regimens, compliance and product purity is pronounced. As the phenolic compounds present within propolis vary based on geographical origin, the bioactivities will also vary significantly making it difficult to define a correct dosage . Nevertheless, there are some studies that can bring asafe dosage range and, as cited above, all propolis types, irrespective of origin and consequently the compounds they contain, have been showing antimicrobial activity indicating it is the collective of propolis compounds rather than the individual compounds that result in its antimicrobial activity. These compounds may be called, as vastly discussed, cationic AMPs and be the focus of propolis analysis. Perhaps, if obtained in an ideal way, all propolis extracts may be effective given that all of them have cationic AMPs - and the way to test the effectiveness may be very simple, as will be further presented.

Considering that most viral infections, mainly in the respiratory tract, with proper care last no more than two weeks, and some effects of propolis on viruses reached efficacy after two weeks of administration ,a safe window to fight viral infections using propolis may be 14 to 21 days, which is a really short time for any complications .

A specific study in type 2 diabetes human patients used a total daily intake of $\mathbf{9 0 0} \mathbf{~ m g}$ (300mg dry extract 3 times a day) of bee propolis supplement for 12 weeks, not reporting side effectsand achieving the intended outcome in the subjects. A trial on mice used a much higher dose of propolis (ethanolic extract) at $200 \mathrm{mg} / \mathrm{kg}$ of body weight (bwt), using as a reference a trial on mice that has previously shown that this dose has antioxidant properties with regard to bone fracture healing. Up to $300 \mathbf{m g} / \mathbf{k g}$ bwt was used in another study on mice, achieving the results with no reports of toxicity or side effects. Although reports of allergic reactions are not uncommon, propolis is relatively non-toxic, with a no-effect level (NOEL) in a 90-mouse study of $1,400 \mathrm{mg} / \mathrm{kg}$ bwt/day . Another study, quoting others, stated that clinical and in vivo studies on animal models have reported that propolis is well tolerated and non-toxic, the NOEL on mice and rats isover $1,470 \mathrm{mg} / \mathrm{Kg} /$ day at 60 days, and over $2,470 \mathrm{mg} / \mathrm{Kg} /$ day at 90 days. In humans, toxic effects occurat dosages as high as $15 \mathrm{~g} /$ day .

Another study in humans, already quoted above, showed a preventive effect on the incidence of respiratory tract infections using in children a solution dose of $5.0 \mathrm{~mL}$ for ages of 1 to 3 years and $7.5 \mathrm{~mL}$ for ages of 4 to 5 years twice daily for 12 weeks, with no reports of side effects, i.e., a daily dosage of $\mathbf{5 0 0 \mathbf { m g }}$ in the first group and of $750 \mathrm{mg}$ in the second. Considering that children of 1 to 3 years weight on average a minimum of $8.8 \mathrm{~kg}$ and 4 to 5 years weight a maximum of $18 \mathrm{~kg}$, which means a tested safe dosage range of approximately $41.6 \mathrm{mg} / \mathrm{kg}$ bwt to $56.8 \mathrm{mg} / \mathrm{kg}$ bwt on children .

In a small pilot study $(\mathrm{N}=18), 20$ drops of an ethanolic preparation of Brazilian green propolis taken 3 times a day for 7 days, a dose usually recommended, was not effective in eradicating H. pylori, despite in vitro studies suggesting inhibition of bacterial growth. Another study that also concluded that propolis is non irritant on the cutaneous membrane, demonstrated that, in conscious mice, themedian lethal dose (LD50) of propolis extract is more than $7.34 \mathrm{~g} / \mathrm{kg}$ bwt, confirming that the product is not toxic and generally safe .

In Brazil the manufacturer recommended dosage of green propolis ethanolic extract, as it is considered a dietary supplement, is usually $20(1 \mathrm{ml})$ to 30 drops $(1,5 \mathrm{ml})$, once or twice a day; or 1 to 2 capsules of 400/500 $\mathrm{mg}$ of dry extract. Considering that Brazilian propolis extract has usually $11 \%$ of dry propolis (11\% w/v), 
the recommended dosage of ethanolic extract as a supplement varies from the minimal daily dosage of $110 \mathbf{~ m g}$ to a maximum of $330 \mathrm{mg}$. On the other hand, the dry extract capsules dosage varies from $400 \mathrm{mg}$ to1,000mg a day.

As mentioned above, like inside the hive and given the physical properties, the applied dosage of propolis to fight microbes is extremely important to the success of the treatment and,fortunately, there is a really safe range to use propolis in humans. The minimal daily dosage to be effective against microbial infections in adults (considering an average weight of $\mathbf{7 0} \mathbf{k g}$ ), based on the facts above and anecdotal evidence, as will be described below, would be at least a total of $1,000 \mathrm{mg} /$ day or an average of $14.2 \mathrm{mg} / \mathrm{kg}$ bwt . In more severe cases, the dosage may be elevated and, as seen above, the limit tested in

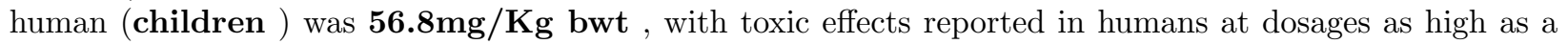
total of $15 \mathrm{~g} /$ day , which means approximately $214 \mathrm{mg} / \mathrm{kg}$ for an adult .

In this way, if necessary, the dosage may be safely elevated up to the limit of $200 \mathrm{mg} / \mathrm{kg}$ of bwt or $14,000 \mathrm{mg}(14 \mathrm{~g})$ daily for an adult weighting $70 \mathrm{~kg}$, through oral administration (not really recommended as seen above, but still useful as more advanced methods are not available yet) and/or inhalation, as will be discussed below (maybe a better way to administer, even if the disease is not in the respiratory tract). It is believed that, in higher doses situations, propolis should be increased gradually and observing any unexpected reactions. Forchildren above 1 year, as already cited above, doses could undoubtedly be safely applied to the maximum of $56.8 \mathrm{mg} / \mathrm{kg}$ bwt . All dosages recommended are immensely far from the median lethal dose (LD50) of $7.34 \mathrm{~g} / \mathrm{kg}$ bwt documented in rodents, which is also an impossible dose for humans to ingest orally through ethanolic extract or even capsules.

Therefore, propolis may be used safely in extremely high amounts . As there are only a few studies regarding dosage and none were found with the specific objective of fighting viruses in humans,it is extremely important, under a health care professional supervision, to test dosage empirically inside the safe range in order to achieve success in fighting these kinds of infections and others.Propolis, as vastly shown, given its possible cationic AMPs,may have a physical and dose-dependent effect, so, although obvious, it is really important to find the necessary and correct dosage and apply it during the necessary time .

\subsection{Pharmacokinetics}

In a review about propolis health benefits, focusing on the phenolic compounds, utilizing some other studies as references, the physiology, digestion and metabolism was described in this way :

Propolis comprises of lipids, waxes and resins in a complex matrix with a large molecular weight, contributing to a poor bioavailability and absorption. The form of polyphenol administered (natural fruit, juice or extract) or the presence of multiple polyphenolics support synergistic effects and are important determinants of bioavailability. Factors believed to contribute to poor bioavailability of polyphenols include digestive instability, poor transcellular efflux in intestinal cells, and rapid metabolism and excretion. Since dietary polyphenols exist as esters, polymers or in glycosylated forms, they cannot be absorbed and must be hydrolysed by the intestinal enzymes or by the colonic microflora before absorption. When in the intestinal system, poorly absorbed polyphenolic compounds are converted to smaller phenolic acids with improved bioavailability, aided by enzyme activity of the colonic microbiota. As microbiota vary between people the inter-individuality in absorption and metabolism is being increasingly considered. Despite poor absorption percentages of bioaccessible phenolic compounds in propolis, the recovered amounts detected in plasma were still high due to their high initial contents compared to other food materials such as fruits and vegetables. The rate at which polyphenols are excreted in the urine appears to vary considerably between individuals. Those placed on a three-day high phenolic diet demonstrated this variable excretion with one participant excreting 8 of the 17 phenolic acids measured, another all 17. The reasons for individual variability in excretion are generally unknown, but may be related to ageing, renal function or inherent propolis properties. Ongoing research and overall consideration to the general health of consumers of propolis and the effect on renal function is warranted. 
Being the main constituents studied, flavonoids (phenolic compounds) are held as the greatest contributors to the pharmacological activities of propolis. Flavonoids from propolis, almost exclusively aglycones, although their antibacterial, antiviral, antifungal, and anti-inflammatory properties, are characterized by low solubility and poor bioavailability and the solubility and oral bioavailability of flavonoids have been reported to be increased by utilizing the phytosome forms and cogrinding technology .

As vastly discussed, AMPs, which are basically amino acid compounds, mainly peptides, are about $40 \%$ of propolis and are believed to be its active ingredient . No studies about the absorption of these components specifically in propolis were found. So, the basics of peptides absorption should be considered and, as already said, always taking into account that propolis peptides may be a little different given their cationic nature and the affinity that they may present with gut bacteria, possibly damaging it. Perhaps the acidic and enzymatic digestion in the stomach can denature theses peptides; however, despite the unfavorable pharmacokinetics, even for phenolic compounds, studies with peroral administration of propolis still show antimicrobial and disease fighting properties, so, some of these peptides may be resistant to digestion. Also, it is controversial if larger peptides (like the ones from bees and plants that have always, at least, more than ten amino acids) are absorbed intact in the gut, but there is little unequivocal evidence that dietary bioactive peptides, other than di- and tripeptides, can cross the gut wall intact and enter the hepatic portal system in physiologically relevant concentrations. There are other studies about methods to enhance peptides absorption through the digestive tract. Another consideration to be done is that, given the detergent-like nature of the compounds, the absorption in the digestive tract possibly can be hindered by food, especially fat rich food, somaybe it is better to take propolis on an empty stomach .

It is worth repeating that, as quoted above, with regard to absorption and excretion, AMPs half-life seems to be high, and some may last for days. They seem to also have better thermal stability and good water solubility, despite the fact that their susceptibility to proteases can be high when ingested ; but with some showing resistance to the hydrolysis of trypsin and pepsin . Again, beyond the digestion problem, it is believed that AMPs may adhere to gut bacteria and cause their death - and if propolis is, as studies have been showing, a strong antimicrobial substance, good or bad bacteria death may be the expected effect, mainly if the effects stem from cationic AMPs, which have a physical/electrostatic mechanism of action without any selection between bacterial species. These effects on gut are not desirable in a sick organism, thus it is believed that propolis parenteral administration (directly or close to the site of the infection/disease) is necessary to keep the best efficacy of the peptides in the substance and to preserve the host as well. Studies are necessary to also understand how these possible cationic AMPs are processed in the kidneys, degraded and excreted. Inside the organism, although no studies on it, the expected effects of AMPs are the ones already extensively studied above. That is, in summary,since they are cationic, AMPs will bind, through electrostatic action, to the diseased cells/invaders and their parts that present an anionic profile - acting also as possible physical/chemical messengers for immunomodulation in host's cells . AMPs, as demonstrated, will be more attracted and active in lower pH, which normally occurs in the infection/disease sites .

As will be exposed below, considering that other means of parenteral administration are still not developed for propolis, a more efficient mean of administration may be through inhalation, especially in the case of respiratory tract illnesses. The method was already tested in vivo and seems to be safe, allowing direct application to the site of infection/disease or close to it. Inhalation also allows a more efficient delivery and absorption of the cationic AMPs throughout the organism to be treated, preserving the gut from possible damage.

\subsection{Pharmaceutical Form}

Regarding pharmaceutical form, in Brazil, the most common is the ethanolic extract $(11 \% \mathrm{w} / \mathrm{v})$ - A recent study with Brazilian red, brown and green propolis found the ethanolic to be better given that the extraction with supercritical fluid was not efficient to obtain extracts with the highest contents of antioxidants compounds. The best results were shown for the extracts obtained through the conventional extraction method (ethanolic) indicating a higher selectivity for the extraction of antioxidants compounds 
and the red variety showed the largest biological potential, which included the content of antioxidants compounds. Another study from Lithuania, stating that propolis is commonly used as ethanolic extract because most of active substances of propolis are ethanol-soluble, concluded that aqueous and nonaqueous propolis extracts differ by composition, but all of them possess antioxidant properties and neutralize $\mathrm{H}_{2} \mathrm{O}_{2}$ in solution at similar efficiency; however, both polyethylene glycol-aqueous and ethanolic propolis extract were more effective in decreasing intracellular and intramitochondrial ROS (reactive oxygen species) compared to aqueous extract. Maybe this occurs, besides phenolic compounds being more soluble in ethanol, due to the fact that propolis extraction process, in order to make it soluble in water, may end up changing or removing some of its hydrophobic components, which possibly harm propolis final synergistic physical effects.

A previous study of the same group of researchers from Lithuania stated that the active substances of propolis are easily soluble in ethanol and, unfortunately, the main biologically active substances of propolis are scarcely soluble in water, oil and other solvents usually used in pharmaceutical industry; however, total amount of phenolic compounds in extracts made in polyethylene glycol and water mixture or in polyethylene glycol, olive oil and water mixture at $70{ }^{\circ} \mathrm{C}$ was comparable to that of ethanolic extract . Investigated non ethanolic extracts (containing polyethylene glycol 400 at 20\%) inhibited the growth and reproduction of all tested microorganisms and the antimicrobial activity of some extracts was equal or exceeded the antimicrobial effect of ethanolic extract; while extracts made in pure water or oil only at room temperature (containing more than 5, 10-fold lower amount of phenolic compounds ) demonstrated no antimicrobial activity. It is worth mentioning that the extracts were rudimentarily extracted in a short time and maybe the ethanol and polyethylene glycol in the other extracts may have contributed greatly to the antimicrobial activities. Besides, the study, as almost all others, was focused solely on the phenolic compounds.

Therefore, considering that the focus of the studies quoted is on the phenolic/antioxidant compounds, the most effective form to accentuate them is the ethanolic extraction of propolis. For this purpose, the variety of types, differences and origins of the substance may be overcome by standardization of the product, like a big Brazilian producer is already doing. In Italy, there is also a patented form of propolis that promises a greater form of absorption of its flavonoids, as already cited. Just to remember, ethanol can possibly denature the peptides in propolis . Propolis extraction methods through acids, alcohols, heat and other physical stressors, as mentioned above, all have the potential to impair the efficacy of the final product (AMPs) and the focus only on its phenolic compounds is surely impoverishing the extraction .

In severe cases, in which a really high dosage may be necessary, a more concentrated ethanolic/aqueous extract solution (in Brazil there is an ethanolic formula with twice the concentration) may work better to reduce the amount of liquid or alcohol ingested. Also, there arecapsules of propolis dry extract ; however, considering that they are less common; the few studies on them and theimpossibility of inhalation ; they will not be considered as an alternative pharmaceutical form for propolis and hence are believed to be not yet recommended. Other pharmaceutical forms for parenteral administration, such as intramuscular, subcutaneous or intravenous, are not common and practically not researched by science - with the exception of the administration through inhalation, which uses the existing extracts and will be discussed below. The only possible alternative pharmaceutical form found was a patent of a propolis extract with improved water solubility that can be used by injection, but it seems that the final product was never developed. It is believed that, in more severe cases, propolis existing extracts are able to be used in hospitals and UCIs through inhalation and through the nasogastric tube.

In Brazil, the minimal legal concentration that must be used in the production of ethanolic extracts is $30 \%$ of dry propolis (in natura) dissolved in $70 \%$ of ethanol/water, which ends up yielding a final solution, after filtering and finishing the process, with about $11 \% \mathrm{w} / \mathrm{v}$ of dry extract (aqueous extracts also follow the concentration of $11 \% \mathrm{w} / \mathrm{v}$ of dry extract in the final product ). The reason for this low concentration was not found. It is so low maybe because of the focus solely on the phenolic compounds, which make it 
difficult to obtain a higher concentration given the wax and other glue-like substances that end up dissolved in the ethanolic extract. These substances in a high concentration are not soluble in water, and as aqueous means are used to dilute and consume propolis extracts, a higher concentration may produce a waxy and indissoluble final product. This is also the reason why it is suggested that a focus on the cationic AMPs (water soluble) and in parenteral administration (to preserve gut flora and also deliver the AMPs intact and in higher concentration) is desirable for the development of pharmaceutical forms.

Once again, it is worth mentioning that the cationic AMPs are not yet explored by science and are believed to be the mechanism responsible for the disease fighting properties of propolis, not the phenolic compounds (maybe only the vehicle/enhancers). Propolis possible main active ingredients work through physical contact (electrostatic action), so,it is believed that the lesser the previous interaction with organisms/agents that the cationic AMPs may bind or be denatured by; the higher will be the power of the substance to fight infections/diseases. Despite most commonly used for propolis extraction, as quoted, the ethanol may denature the peptides - in the experiment of breaking of the surface tension of water developed in the present work, propolis aqueous extract showed a slightly stronger effect, perhaps indicating that the aqueous extraction may be better.

In this way, it is speculated that the pharmacological extraction and concentration methods are still far from the full benefits that this extraordinary substance has to offer and advances are necessary - which does not seem difficult if the focus is altered to the AMPs. In regards to the parenteral means of administration, further research and advances are extremely necessary as well. However, as exposed, the existing extracts, ethanolic or aqueous, regardless of the origin, may be able to provide strong electrostatic effects and the effectiveness may be easily tested. The tests can be similar to the experiments developed in the present study (especially through breaking of the surface tension of water), in order to measure if the extracts have a minimum desired surfactant/detergent property. If this property is satisfactorily present, regardless of the mean of presentation, it is believed that the extract is able to successfully fight microbes/diseases. It is worth remembering that the extracts should be applied directly or close to the site of the infection/disease, in the correct dose and for the sufficient time it is a "detergent" that needs the critical micelle concentration (CMC). The most important issue is to correctly extract the "whole immune system from bees and implant it intact in the infection site "in the necessary amount, which is believed to be what the studies from now on will have to focus, increasing the cationic AMPs concentration in the extracts to enhance their effects.

\subsection{Interaction with other Drugs and Possible Resistance}

One study already raised the possibility of interactions between Brazilian Green propolis and therapeutic drugs metabolized by the enzyme CYP1A2, of the cytochrome P450 family, such as duloxetine . However, another study, in humans, using a standardized Brazilian Green propolis extract (not only phenolic compounds) found that it did not clinically change CYP1A2, CYP2C9, CYP2C19, CYP2D6 and CYP3A activities, since, despite statistical significance, the magnitude of the changes in plasma concentration (AUC) values after administration were all below $20 \%$ and, therefore, may be considered safe regarding potential interactions involving these enzymes. It is also unlikely that propolis will antagonize the activity of reverse-transcriptase inhibitors (RTI) such as AZT or protease inhibitors (PI) such as indinavir . An important consideration is that, as many propolis preparations contain high level of alcohol, they may cause nausea when taken with metronidazole .

No other studies about possible drug interaction were found, thus, on the other side, as a strong example of the low possibility of harmful interactions of propolis with other medications, mainly antiinflammatory and glycemic/lipidic control drugs, it can be mentioned two trials already exposed above. The studies were developedusing propolis to treat type 2 diabetic humans, whichusually are under treatment using a variety of medications and are more susceptible to acute inflammation complications. In both,none of the participants presented adverse effects but showed, in fact, the improvement of antioxidant function, modulating the inflammatory response to chronic inflammation, and demonstrating improvement of glycemic and some serum lipid levels . 
In addition, as already commented above, another study preliminarily showed no side effects, but beneficial synergism between propolis ethanolic extract and antimicrobial drugs . These interactions occurred especially with agents that interfere on bacterial protein synthesis such as choramphenicol, gentamicin, netilmicin, tetracycline and vancomycin. Besides this, the absence of antagonism between propolis ethanolic extract and all drugs tested, brought the hypothesis of the potential medical use of propolis in combination with certain antimicrobial drugs on staphylococci diseases, since bacteria may be resistant to several antimicrobial drugs. Other demonstration of propolis synergistic effects was a study confirming significant results in combinations of cefixime and propolis as compared to infected controls, concluding that propolis acted synergistically with cefixime and enhanced the efficacy of the antibiotic, reducing the antibiotic effective dose in combined therapy.

As seen, the cationic AMPs from propolis may be similar to AMPs from all innate immune systems that, despite all the biochemical complexity, evolved in a really similar manner, enabling them to "connect" (even with the adaptive; AMPs are possible physical/chemical messengers as well) and work together against pathogens/diseases - besides direct effects, AMPs also promote and modulate host's own defenses. Consequently it is believed that these AMPs possibly will not interact with drugs differently from the host's own AMPs, i.e., propolis may be safe to be used with most existing drugs - even immunosuppressants, given the immunomodulatory effects of propolis. Further studies are needed to extensively research the mechanism of action and possible interactions, focusing especially in the peptide contents of propolis.

Regarding possible microbial resistance against propolis, no studies were found specifically about it, but, as extensively seen, some studies show, directly or indirectly, the opposite, that propolis is designed to not be resisted . If definitely proven that propolis works - especially its cationic AMPs -, it is designed to achieve practically $100 \%$ of efficacy in $100 \%$ of cases given that the effects are physical/structural rather than biochemical/functional - remembering that there may be three other important not so obvious variables involved in the equation: dosage, site, and time. As the subject was amply discussed above, it will not be discussed again. It is worth mentioning that, as propolis main active ingredients are believed to be the cationic AMPs, they usually eliminate microbial pathogensby attacking their membranes and cell wall constituents andresistance to AMPs has so far not been observed, which makes them attractive candidates for the development of new antibiotics in human medicine. Further studies are necessary in all areas as the possible existence of a large amount of cationic AMPs in propolis is a totally new field. For now, thecombination of propolis with other drugs should be oriented and supervised by health care professionals .

\subsection{Adverse Effects}

As already mentioned above, another singular property described in clinical investigation, in mice and humans, is that propolis and its constituents are generally well tolerated and non-toxic, unless administered in very large quantities. It was found only an anecdotal evidence of kidney failure and some indications that propolis may decrease kidney perfusion; however, it was a long term treatment, the patient studied had a serious comorbidity and the kidney function improved after propolis withdrawal . In comparison to other antimicrobial and anti-inflammatory drugs, the lack of adverse effects of propolis is notorious, despite some allergic reactions, mainly in children and adolescents, that can be easily overcome with asimple patch test before prescribing. Tests in children have been demonstrating propolis safety, even in small children. Actually, as already commented, tests in murine asthma showed an inhibition of both the inflammatory cells migration to the alveolar space and the systemic progression of the allergic inflammation, demonstrating that propolis may also act as anti-allergic agent .

All these properties and the general absence of side effects may help the infected organism to preserve and restore the homeostasis, not putting more stress on the overburdened immune system, but, on the contrary, enhancing host's general ability to fight the inflammationcaused by microbial 
infection or other diseases. According to some studies focusing only on the phenolic compounds, propolis has some demonstrated probiotic nature and may have some benefic effects on intestinal barrier function, but overall, the degree that propolis supports the health of the intestinal microflora has been poorly investigated with longer term studies needed on the effect of dietary polyphenols on gut microbiota, an area worthy of further research . In general, comparing to antimicrobial drugs, it is believed that propolis may cause less adverse effects on gut health given its immunomodulatory properties. However, it is also believed that, as said, as a potent antimicrobial, although a polyphenol-rich substance, propolis may not be recommended for the treatment of long term gut problems as it may disrupt the balance of the gut natural microbiome - especially because of the possible large amount of cationic AMPs in propolis, with a high affinity for any bacteria.

Again, as a strong example of the inexistence of adverse effects in the administration of propolis in humans, it can be cited the two studies already exposed that were developed with type $\mathbf{2}$ diabetic humans - which are usually under treatment with a variety of medications and are more susceptible to acute inflammation complications. The trials demonstrated no adverse effects and the improvement of antioxidant function, with modulation of the inflammatory response to chronic inflammation and the improvement of some glycemic and serum lipid levels. As seen and must be repeated, the cationic AMPs from propolis may be similar to AMPs from all innate immune systems that, despite all the biochemical complexity, evolved in a really similar manner, enabling them to "connect" (even with the adaptive; AMPs are possible physical/chemical messengers as well)and work together against pathogens/diseases - besides direct effects, AMPs also promote and modulate host's own defenses. Hence AMPs are not meant to cause adverse effects. Some recent studies, always focusing on phenolic compounds, for example, compared the effects of propolis extracts in normal and cancer cells, with mixed results about the possible cytotoxicity in normal cells . Thus, further studies specific about possible cytotoxicity are necessary, mainly in vivo; however, if existent, the cytotoxicity is believed to be low because propolis has been extensively used to treat several diseases, especially through direct application - e.g., in wounds, showing regenerative properties and accelerating the healing process .

An important way to diminish adverse effects and enhance the action, as already said, may be the parenteral administration of propolis, which should be further studied. Also, it is important to remember thatmost propolis extracts contain alcohol ; so, adverse effects derived from this component, even in small amounts, should be considered. As cited above, in humans, toxic effects of propolis occur at dosages as high as $15 \mathrm{~g} /$ day . Considering that all studies so far focused only the phenolic compounds, further detailed studies to deeply understand the cationic AMPs of propolis should be done to allow a better understanding of their possible adverse effects. For now, empirical knowledge has been showing low to none adverse effects of propolis. It is worth mentioning thatpossible allergies to propolis should be tested and other adverse effects monitored by health care professionals, especially for patients with comorbidities and/or under medication . Lastly, before prescription, an easy patch test - or other sort of allergy tests that may be considered necessary given the patient history - may be enough to avoid most of the possible adverse effects and, in case of doubt, the dosage may be initially small and be slowly increased to observe any adverse effects .

\section{Propolis Inhalation}

Beyond the beneficial effects on the lungs brought by means of peroral administration of propolis, which were already vastly mentioned and stressed throughout the present study, propolis may be a powerful therapeutic agent to be directly applied to the respiratory tract. Also, besides the focus only in the phenolic compounds and not in the cationic AMPs, it was found that all existing works about propolis possible internal effects are mostly in vitro, with only a few studies in vivo approaching the systemic effects of propolis in infected organisms. Almost none of them are about the effects of propolis when it is applied directly to the site of infection/disease, which may be extremely important and the key piece for the achievement of the full efficacy of the substance in the treatment of most diseases. 
One of the only two existing studies found about propolis inhalation evaluated whether Brazilian green propolis inhalation can inhibit Dermatophagoides farina induced airway hyperresponsiveness (AHR), eosinophilic infiltration and other histological changes in the lung ( $\mathrm{T}$ helper 2 cells, cytokine production and airway remodeling) in a mice model of asthma. The trial demonstrated that treatment with propolis significantly reduced the levels of several cytokines (IL-5, IL-13, eotaxin, MCP-1, and TGF- $\beta 1$ ) in bronchoalveolar lavage fluid. The goblet cell metaplasia, thickness of airway smooth muscle, and airway fibrosis were markedly decreased in propolis-treated mice. Furthermore, AHR to acetylcholine was significantly abrogated in propolis-treated mice. The study suggested that the results indicate that propolis has a potential to reduce airway remodeling and AHR in asthma model and may potentially be beneficial as a prophylactic and therapeutic agent for asthma . The study in question did not report adverse effects, and, although only one, it is believed to be a strong indicator thatpropolis may be really powerful when applied directly to the disease site .

The other study about propolis inhalation was done with humans (the work was quoted in another article and, unfortunately, access to the full content of the research used as reference was not possible). In the trial, using propolis from Poland, a total of 260 steel workers suffering from bronchitis were treated for 24 days by various methods, including local and systemic regulation of the immune system and local treatment with an ethanolic extract of propolis (EEP) in a physiological salt solution. The best results were obtained in patients treated with EEP inhalations. In India, an article generically quoted that people commonly use inhaled propolis in the treatment of respiratory tract diseases, without details of how it is done; also quoting that propolis is inhaled through an oil emulsion to treat asthma, pneumonia and other respiratory diseases in children. As can be noted, there are very few studies developed in regards to this method of parenteral administration by means of propolis inhalation. Thus, further research is definitely needed as it seems a promising method of treatment of a broad range of respiratory tract diseases - as mentioned above, from asthma to cancer -, allowing propolis to be applied directly to the infection/disease site, which is believed to be quite important.Perhaps this mean of administration, given the powerful mechanism and effects of propolis demonstrated in the present study,is ready to be used as a treatment alternative or an adjuvant, not just for lung cancer but for all types of cancers .

Once again, as the cationic AMPs from propolis have physical/electrostatic action, direct application to the infection/disease site - in the case of respiratory tract diseasesdirectly in the respiratory tract through inhalation - allows the direct contact of these AMPs with the affected tissues/invaders and their products and substances. This contact produces direct effects on the site, with propolis positively charged AMPs physically binding to all the parts in the structure of the microbes/host's cells and their products/substances that have negative charges, potentially annihilating the invaders, and modulating the host immune system as well . It is speculated that inhalation, in theory, is also ableto preserve the synergy of the compounds existing in propolisand reach bioequivalent effects of direct applications of propolis to microbes in vitro, especially in the case of respiratory tract infections. In viral infections, for example, as quoted, AMPs have, integrating in either the viral envelope or the host cell membrane, basically four antiviral mechanisms: (a) viral envelopes disruption; (b) viral receptors blocking; (c) preventing viral particles from entering host's cells by occupying specific cell receptors; (d) crossing the cell membrane and in the cytoplasm and organelles, cause changes in the gene expression profile of the host cells, helping them fight against viruses or block viral gene expression. Another two possible advantages of propolis inhalation are the fact that the substance and its cationic AMPs have possibly high adherence (may facilitate the adhesion and spreading throughout the large surface of the lungs) and, as seen, various volatile compounds that may also have antimicrobial properties .

In addition, it is believed that direct administration through inhalation is able to increase delivery and absorption of the active ingredients (AMPs) throughout the entire organism, enhance systemic effects (immunomodulatory, furthering host's homeostasis), and diminish adverse effects on the digestive tract. In fact, the high surface area and high permeability of the lungs make them an ideal site for 
rapid systemic delivery of macromolecules - and they can be delivered without injections, e.g., an inhaled insulin product already in use - and small-molecule drugs, which are absorbed more rapidly through the lungs than through the gastrointestinal tract, with higher bioavailabilites and reduced first-pass metabolism by enzymes. The lungs are significantly permeable to many peptides and proteins, with the rate of absorption decreasing with increasing molecular mass. Small peptides and proteins are absorbed more rapidly after inhalation than after subcutaneous injection - For other small molecules, inhalation is also a fast way to get into the body because drug efflux transporters and metabolizing enzymes are present in the lung at much lower levels than the gastrointestinal tract . Lipophilic small molecules are absorbed extremely fast, $\mathrm{t}_{1 / 2}$ (abs)approximately 1 to 2 minutes ; water-soluble small molecules are absorbed rapidly $t_{1 / 2}$ (abs) approximately 65 minutes. Small molecules can exhibit prolonged absorption if they are highly insoluble or highly cationic .Concentrations of AMPs in lung secretions are altered in several pulmonary diseases, thus, the cationic AMPs from propolis may be a key piece, acting in synergy with host's AMPs . There is also atendency towards applying aerosolized antibiotics, which can actually help to eradicate multidrug-resistant organisms . Optimizing distal deposition of aerosolized antibiotics in the lungs in patients with hospital-acquired or ventilatorassociated pneumonia is the key to minimizing intensive care unit contamination and risk of development of resistant pathogens.

A favorable consideration for propolis inhalation as well, is the fact that propolis, as already exposed, is generally well tolerated in all tissues, internal or external, and studies have been vastly demonstrating it. Thus, in the respiratory tract epithelium, propolis may not cause any adverse effects, but perhaps the opposite, as the studies have been showing, furthering immunomodulation. If no allergies exist, it is believed that, despite studies are still necessary, propolis may be safely inhaled . Cationic AMPs are, as said, totally soluble in water, so, they are easily dissolved and nebulised .

Regarding the procedure, it is suggested that the inhalation should be done by combining propolis extract (ethanolic or aqueous) withsterile $\mathbf{0 . 9 \%}$ saline solution (the vehicle normally used for inhalation; it is believed that, as the experiments indicate, the low concentration of salt in the saline solution is unlikely to affect the efficacy of propolis) in a proportion of approximately $\mathbf{5 \%}$ of the extract in the final solution (with an $11 \% \mathrm{w} / \mathrm{v}$ commonly found propolis extract, it means $0.55 \% \mathrm{w} / \mathrm{v}$ of dry extract in the final solution). That is, one drop of propolis extract for each $\mathbf{m l}$ of saline solution (20 drops yield approximately $1 \mathrm{ml}$ of propolis, so, if added in 19-20 ml of solution, will end up in a percentage of aprox. $5 \%$ of the final solution). This is believed to be an amount that will not cause any burning in the skin or stinging effects in the respiratory tract, keeping the freshness and revitalizing effects of the solution. Thus, if more is necessary, it is recommended to extend the time of exposition, raising the total amount of solution, instead of altering the concentration of it . However, the tolerance is variable and the concentration should be personalised, always remembering that theeffects of propolis are dosedependent, so the dosage must be applied in reasonable amounts . For the inhalation, aprofessional or domestic inhaler device can be used - domestic devices (nebulizers) usually are pneumatic (ultrasonic can be used as well) and have approximately 50 PSI and $0.4 \mathrm{ml} / \mathrm{min}$ nebulization rate, taking approximately $50 \mathrm{~min}$ to finish an inhalation of $20 \mathrm{ml}$ of solution. It is worth stressing that, as the absorption of propolis may be greater through the respiratory tract, in case of adverse reactions the daily dosage should be adjusted - as said, propolis dosage may be extremely high, with low toxicity; so, it is believed that, if no allergies exist, adverse effects will be rare in inhalation.

As a final observation, it is worth mentioning that, despite the direct and immunomodulatory effects, another yet to be studied effect that may accrues from propolis unexplored AMPs, as strong surfactants, is the possible synergy with lungs natural surfactants, improving breathing and producing another broad range of beneficial effects on the local immunity. These may be highly desirable effects in the treatment of acute respiratory diseases. Studies are necessary to explore this possible and also extraordinary property, which, as quoted above, may be used for the treatment of premature babies and other chronic respiratory diseases. A study in rats, e.g., demonstrated that inhalation of aerosolized pulmonary surfactant could significantly reduce lung injury . 
In conclusion, as already exposed, in a simple manner, propolis seems to be basically a more complex and selective/non-toxic "detergent/soap" that can be safely used internally and consumed by humans with no side effects (especially if compared to regular detergents), and inhalation may enhance this property. Analogies between membrane-active peptide and detergents have been studied, but AMPs, as discussed, demonstrated that, besides amphiphilic detergent/soap-like properties, they can be more powerful on viruses and other microbes than any existent detergent given its especial properties - not just involving them, but through a broad range of other mechanisms. Moreover, propolis inhalation may be extensively used as a prophylactic measure for those exposed to possible pathogens, such as health professionals dealing with highly contagious diseases, allowing them to literally "wash their respiratory tract" with a powerful and non-toxic "soap/detergent" . It is like "implanting" a whole immune system inside the respiratory tract, which also further host's defense actions .It is also like "enveloping" the lung and transforming it in a "hive", protecting against any pathogens or diseases . Even in internal diseases, out of the respiratory tract, it is believed that the inhalation is the best way to administrate propolis so far as no other parenteral methods are available, and, as said, the method is able to spare patient's gut microbiota from damage and enhance systemic absorption. Perhaps an alternative or adjuvant treatment for several diseases is ready to be extensively used, with further studies needed, especially to improve and concentrate the propolis extracts used.

\section{Possible Effects of Propolis against COVID-19}

\subsection{Facts about SARS-CoV-2}

SARS-CoV-2 is still a relatively new sort of virus; therefore, studies on it are controversial, firm consensus inexistent and new information about the virus can become obsolete in days given the current pandemic and the large amount of studies that are being produced. It is yet not even known: if the virus is capable of mutation or recombination, hindering vaccines development and immunity; if the amount of virus exposure affect the severity of the disease; if the host, once infected, become immune or not and how long the immunity may last; if the virus is able to become a persistent infection, staying in the body and resurging; or, lastly, if the disease is able to become endemic or not . The severe acute respiratory syndrome coronavirus 2 (SARS-CoV-2), previously known as 2019 Novel Coronavirus (2019-nCoV), is apositive-sense, singlestranded RNA virus that causes the potentially lethal COVID-19 respiratory tract infection. This new virus belongs to the genus Betacoronavirus, which also includesSARS-CoV and MERS-CoV . The first case of COVID-19 was detected in December, 2019 in Wuhan, China, and has been recently declared a pandemic. Human-to-human spread has been confirmed, with a suspected incubation period of $\sim 2-14$ days. There are some reports of transmission in the absence of clear symptoms, though infected people are likely most contagious due to coughing and sneezing that expel respiratory droplets. SARS-CoV-2 is an enveloped virus with a genome comprising 29,891 nucleotides, which encode the 12 putative open reading frames responsible for the synthesis of viral structural and non-structural proteins. A mature SARSCoV-2 has four structural proteins, namely, envelope (E), membrane (M), nucleocapsid (N), and spike (S)10.All these proteins may serve as antigens to stimulate neutralizing antibodies and increase CD4+/CD8+ T-cell response .

COVID-19 has a case-fatality rate of $2.3 \%$, with higher rates amongelderly patients and patients with comorbidities . Person-to-person transmission is efficient, with multiple clusters reported. Clinically, patients with COVID-19 present respiratory symptoms very similar to the presentation of other respiratory virus infections. Radiologically, COVID-19 is characterised by multifocal ground-glass opacities, even for patients with mild disease. Knowledge of virus dynamics and host response are essential for formulating strategies for antiviral treatment, vaccination, and epidemiological control of COVID-19. However, a systematic study on these aspects has not been done. There are reports of high viral load that peaks in the first week of the disease, which has practical implication. The high viral load during the early phase of illness suggests that patients could be most infectious during this period, and it might account for the high transmissibility of SARS-CoV-2. Furthermore, this high viral load suggests that SARS-CoV-2 
could be susceptible to the emergence of antiviral resistance . SARS-CoV-2 and SARS-CoV belong to the coronavirus family andboth invade target cells through angiotensin converting enzyme (ACE2) . An in-depth understanding of ACE2 and a series of physiological changes caused by the virus invading the human body may help to discover and explain the corresponding clinical phenomena and then deal with them timely. In addition, ACE2 is a potential therapeutic target .

The analyses clearly show that SARS-CoV-2 is not a laboratory construct or a purposefully manipulated virus and, given the similarity of SARS-CoV-2 to bat SARS-CoV-like coronaviruses, it is likely that bats serve as reservoir hosts for its progenitor. A study of cultured bat cells shows that their strong immune responses, constantly primed to respond to viruses, can drive viruses to greater virulence. Modelling bat immune systems on a computer, the researchers showed that when bat cells quickly release interferon upon infection, other cells quickly wall themselves off.This drives viruses to faster reproduction. The increased virulence and infectivity wreak havoc when these viruses infect animals with tamer immune systems, like humans. It is known that SARS-CoV-2 causes a cytokine storm in the host . It is an excessive immune response to external stimuli and the pathogenesis is complex. The disease progresses rapidly, and the mortality is high. Certain evidence shows that, during the COVID-19 epidemic, the severedeterioration of some patients has been closely related to the cytokine storm in their bodies. Acute respiratory distress syndrome (ARDS) and multiple-organ failure occurred rapidly, resulting in death within a short time. Cytokine storm is considered to be one of the major causes of ARDS and multiple-organ failure. It plays an important role in the process of disease aggravation. Clinical studies have detected a cytokine storm in critical patients with COVID-19. Therefore, effectively suppressing the cytokine storm is an important way to prevent the deterioration of patients with COVID-19 infection and save the patients' lives . It seems that complications in COVID-19 disease, maybe given the aggressivity of the virus and the high inflammation caused, are also stemming from secondary bacterial infections. Viral infections increase pneumococcal adherence to the local epithelium, facilitating bacterial infection. Adhesion of Streptococcus pneumoniae to epithelial cells, for example, is significantly enhanced by human coronavirus HCoV-NL63 infection.Coronavirus causes inflammatory damage in the lungs, preventing clearance of bacteria . Secondary bacterial infection worsens prognosis.Most deaths in the influenza pandemics of 1918, 1957, and 1968 were caused by secondary bacterial infections. Concurrent bacterial pneumonia was highlighted as a particular problem in elderly people in the 2003 SARS outbreak .

In summary, as seen, SARS-CoV-2 presents four main characteristics: (a) it is enveloped ; (b) it has a high virulence with a high initial load and high replication rate, which could lead toantiviral resistance; (c) it invades cells using ACE2 and possibly other receptors ; and (d) it is highly inflammatory (cytokine storm) and may cause secondary bacterial infection and other effects

\subsection{Possible Effects of AMPs from Propolis on SARS-CoV-2}

As already mentioned above, in viral infections, AMPs have, integratingin either the viral envelope or the host cell membrane, basically four antiviral mechanisms: (a) viral envelopes disruption; (b) viral receptors/proteins blocking; (c) preventing viral particles from entering host's cells by occupying specific cell receptors; and (d) crossing the cell membrane and in the cytoplasm and organelles, cause changes in the gene expression profile of the host cells, helping them fight against viruses or block viral gene expression. Also, these peptides often have a high affinity for bacterial products, such as LPS, allowing them to modulate the host response and reduce the inflammatory response in sepsis . More recently, they have been found to interact directly with host's cells to modulate the inflammatory process and innate defenses. Thus, the cationic AMPs from propolis, especially if applied through inhalation directly to the infection site in the necessary amount have possible powerful promising effects directly on SARS-CoV-2 and in the host immune system, helping to fight the disease. As demonstrated, all the effects are possibly physical, through electrostatic binding, with no possible resistance if applied in the correct dosage. If definitely proven that propolis cationic AMPs 
work, they are designed to achieve practically $100 \%$ of efficacy in $100 \%$ of cases given that the effects are physical/structural rather than biochemical/functional .

Although further studies are necessary - especially randomized trials -, by utilizing the findings already discussed throughout the present work, it is possible to safely hypothesize the effects of the cationic AMPs of propolis in the treatment of COVID-19. Thus, the possible specific effects of the AMPs in question on SARS-CoV-2 will be detailed below according with the four main characteristics of the virus stressed in the previous section and the AMPs four antiviral mechanisms quoted in the paragraph above. In the end, possible effects of the cationic AMPs of propolis on SARS-CoV-2 secondary consequences will be approached as well.

7.2.1 Possible Effects of the Cationic AMPs of Propolis on SARS-CoV-2 Viral Envelope, Viroporins and Enzymes (viral envelope disruption and viral receptors/proteins blocking)

Effective antivirals have been developed against specific viruses, such as HIV, hepatitis C virus and influenza virus. This 'one bug-one drug' approach to antiviral drug development can be successful, but it may be inadequate for responding to anincreasing diversity of viruses that cause significant diseases in humans. The majority of viral pathogens that cause emerging and re-emerging infectious diseases are membrane-enveloped viruses, which require the fusion of viral and cell membranes for virus entry. Therefore, antivirals that target the membrane fusionprocess represent new paradigms for broad-spectrum antiviral discovery. In this class, virolytic antiviral peptides (AVPs) are reminiscent of the broad-spectrum and pleiotropic antimicrobial peptides (AMPs) that are part of the innate immune defence mechanisms present in all kingdoms of life. To date, the best-characterized AVPs are generally small $(2-5 \mathrm{kDa}) \varsigma \alpha \tau \iota 0 \nu\llcorner\varsigma, \alpha \mu \pi \eta \iota \pi \eta \iota \lambda \iota \varsigma \alpha-\eta \varepsilon \lambda \iota \varsigma \alpha \lambda \pi \varepsilon \pi \tau \iota \delta \varepsilon \varsigma$, in which the activity probably relies on their interfacial hydrophobicity. Indeed, AVPs engage in electrostatic and/or hydrophobic interactions with the hydrophobic surfaces of fusion proteins that are transiently exposed during the fusion process, but AVPs also interact with membrane lipids . For example, some cationic, amphiphilic antiviral peptides (AVPs) have detergent-like properties at high concentrations and can result in the formation of pores or lead to the micellization of viral membranes . A review about MERS-CoV, in the same genus of SARS-CoV-2, quoted AMPs as excellent candidates as novel therapeutic agents since they

have been reported to possess anti-coronavirus activity .

In this way, regarding the first characteristic of SARS-CoV-2 showed above that is the presence of a viral envelope, the cationic AMPs from propolis, as seen, may bind to membrane lipids (and also other negatively charged parts) and act as a detergent (again, in the necessary dosage, concentration) causing the micellization of viral membranes and hence avoiding the attachment and replication of the virus. As already mentioned, in a simple manner, propolis seems to be basically a more complex and selective/nontoxic "detergent/soap" that can be safely used internally and consumed by humans with no side effects (especially if compared to regular detergents). Analogies between membrane-active peptide and detergents have been studied, but AMPs, as shown, demonstrated that, besides amphiphilic detergent/soap-like properties, they can be more powerful on viruses and other microbes than any existent detergent given its especial properties, not just involving them but through a broad range of other mechanisms. Propolis may also be used as astrong prophylactic measure, especially by health professionals .

As cited above, the other possible mechanism is the engagement of AMPs in electrostatic and/or hydrophobic interactions with the hydrophobic surfaces of fusion proteins that are transiently exposed during the viral fusion process. So, besides the effects on the membrane, a review about viral envelope , despite about coronaviruses in general - and SARS-CoV-2 is very similar to the others -, described that coronaviruses viroporins are viral-encoded membrane pore-forming proteins that can modulate cellular ion channels and have been suggested to regulate and function in multiple stages of the viral life cycle , from viral entry to assembly and release, and even pathogenesis. Although viroporins are not essential to 
viral replication, their absence does weaken or attenuate the virus and diminishes its pathogenic effects . They tend to be small proteins ( $60-120$ amino acids) of a predominantly hydrophobic nature that oligomerise in the membranes of infected cells, forming hydrophilic pores. Viroporins can transport different ions butappear to be largely selective for the positively charged ions, so, the preference simply appears to be for cations over anions. Hence it is believed that the cationic AMPs from propolis ,taking advantage of the preference for cations, may easily bind to these viroporins and weaken or attenuate SARS-CoV-2 anddiminish its pathogenicity - viral receptors blocking, the second general effect of AMPs on virus mentioned above. In a dangerous virus like SARS-CoV-2, any mechanism that may be able to slow its pathogenic effects is highly desirable .

Beyond the effects above in the membranes and viroporins that are also able to diminish the virulence and replication, a pre-print study about systematic drug repurposing to identify promising inhibitors against SARS-CoV-2 3C-like Proteinase (3CLpro) and 2'-O-Ribose Methyltransferase (2'-O-MTase), due to their indispensable nature in the viral life cycle, produced interesting findings. 3CLpro is a cysteine protease responsible for the proteolysis of replicase polyproteins resulting in the formation of various functional proteins, whereas 2'-O-MTase methylates the ribose 2'-O position of the first and second nucleotide of viral mRNA, which sequesters it from the host immune system . According to the resultsobtained from electrostatic surface potential, all of the drug molecules were found interacting mostly with the negatively charged residues of both 3CLpro and 2'-OMTase, which revealed that these molecules were oriented in the active site of both the proteins. Thus, as the preliminary study demonstrated, also with 3D images, 3CLpro and 2'-O-MTase, which are indispensable to SARS-CoV-2, have extensive negatively charged active sites. These sites mayfurther the binding of the cationic AMPs from propolis, which may inactivate 3CLpro and 2'-O-MTase and hence diminish the high virulence and high replication rate of the virus .

Lastly, a pre-print study also showed that SARS-CoV-2 3CLpro has 96\% sequence similarity to SARS-CoV 3CLpro, with identicalnegatively charged amino acids, which, again, may further the binding of the cationic AMPs from propolis to the protease in question.

7.2.2 Possible Effects of the Cationic AMPs of Propolis on SARS-CoV-2 High Virulence and High Replication Rate, Binding to Host's Cell Receptors HSPG, Heparin and ACE2 (preventing viral particles from entering host's cells by occupying specific cell receptors)

Studies have been revealing that SARS-CoV-2 is very similar in structure and pathogenicity to SARS-CoV, with only a slightly different spike (S) protein . SARS-CoV envelope proteins may havepositive charges interacting with negative charges on the heparan sulfate proteoglycans present on the surface of target cells . As already mentioned above, besides disruption of viral envelopes and blocking viral receptors, some antiviral AMPs can prevent viral particles from entering host's cells by occupying specific receptors on mammalian cells . $\Phi_{\circ} \rho \epsilon \xi \alpha \mu \pi \lambda \epsilon$,

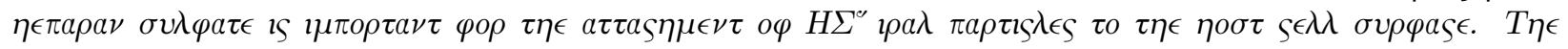

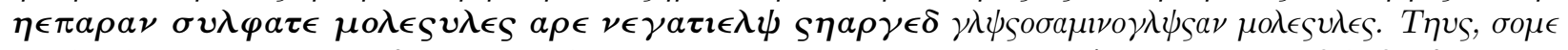

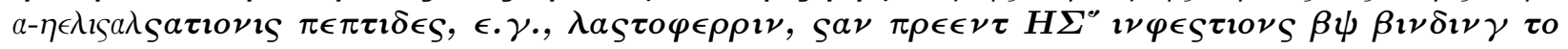

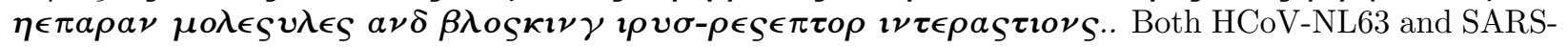
$\mathrm{CoV}$, which use ACE2 as an entry receptor, also utilize Heparan sulfate proteoglycans (HSPG) as attachment receptors. SARS-CoV-2, which also uses ACE2 for cell entry, may also use HSPG as attachment receptors, although no data on this topic is available yet. In this way, given the similarity and the study that will be quoted below about the use of heparin, it is really possible that SARS-CoV-2 uses the same attachment mechanism of SARS-CoV and the AMPs from propolis, as all cationic AMPs, are possibly able to bind to HSPG negatively charged molecules and avoid SARS-CoV-2 attachment to host's cells through this receptor .

Another recent pre-print demonstrated that SARS-CoV-2 spike protein receptor binding domain undergoes conformational change upon heparin binding. Mucosal epithelia such as that of the respiratory tract are protected by a layer of mucin polysaccharides, which are usually sulfated. Glycosamino- 
glycans are ubiquitously present on almost all mammalian cells and this class of carbohydrates are central to the strategy employed by coronaviridae to attach to host's cells. Hence the interaction between the SARS-CoV2 spike protein receptor binding domain (SARS-CoV-2 S1 RBD) and pharmaceutical heparin was measured. The data show that SARS-CoV-2 S1 RBD binds to pharmaceutical heparin and that upon binding, asignificant structural change is induced . Heparin, a member of glycosaminoglycans family, has previously been shown to inhibit SARS-associated coronavirus strain HSR1 cell invasion and this, in concert with the data presented by the study, suggests the utilisation of glycosaminoglycan-derived pharmaceuticals against SARS-associated coronavirus . This study strongly supports therepurposing of heparin and its derivatives as antiviral agents, providing a rapid countermeasure against the current SARS-CoV-2 outbreak. The study suggests as well the parenteral use, direct in the respiratory tract via nasal administration, using nebulised heparin - Although plausible, the study points out only one mechanism of attachment of the virus, which may be overcome by using other receptors and mechanisms to attach to host's cells.

Therefore, in a simple manner, to not describe unnecessary details, as quoted, respiratory tract epithelial cells contain polysaccharides such as heparin, which is one of the strategies employed bycoronaviridae to attach to host's cells . It is known thatheparin - a host cell receptor for different substances, especially proteins - has the highest negative charge density of any known biological macromolecules, which is the result of itshigh content of negatively charged sulfo and carboxyl groups . Clearly the most prominent type of interaction between heparin and a protein is ionic, in which clusters of positively charged basic amino acids on proteins form ion pairs with spatially defined negatively charged sulfo or carboxyl groups on the heparin chain . Again, the cationic AMPs from propolis may be able to bind to heparin negatively charged molecule and avoid SARS-CoV-2 attachment to host's cells through this receptor.

Lastly, regarding the angiotensin converting enzyme (ACE2), as already mentioned, to spare time with details that are not useful, both SARS-CoV-2 and SARS-CoV belong to the coronavirus family and bothinvade target cells through angiotensin converting enzyme (ACE2). An in-depth understanding of ACE2 and a series of physiological changes caused by the virus invading the human body may help to discover and explain the corresponding clinical phenomena and then deal with them timely. In addition, ACE2 is a potential therapeutic target . Considering that the surface of ACE2 is highly negatively charged, a study in 2005 about SARS-CoV spike (S) glycoprotein, speculated that the electrostatic interaction between the viral envelop and the receptor is essential for mediating viral entry . It is believed that, given the similarities already exposed, this electrostatic mechanism is also the same for SARS-CoV-2 and the cationic AMPs from propolis may strongly bind to ACE2 highly negatively charged surface and avoid SARS-CoV-2 attachment to host's cells through this receptor.

7.2.3 Possible Effects of the Cationic AMPs of Propolis on the Cytokine Storm Caused by SARS-CoV-2 (crossing the cell membranes and helping the host)

With a focus on the phenolic compounds, a review quoted above stated that the most important property of propolis is its general anti-inflammatory activity, which is not chemically full understood, through five main demonstrated mechanisms: (a) the inhibition of cyclooxygenase (COX) and consequent inhibition of prostaglandin biosynthesis; (b) free radical scavenging - powerful antioxidant effects of polyphenols and flavonoids; (c) inhibition of nitric oxide synthesis; (d) reduction in the concentration of inflammatory cytokines; and (e) immunosuppressive activity . Just to remember, a study using inhalation demonstrated that treatment with propolis significantly reduced the levels of several cytokines (IL-5, IL-13, eotaxin, MCP-1, and TGF- $\beta 1$ ) in bronchoalveolar lavage fluid .The goblet cell metaplasia, thickness of airway smooth muscle, and airway fibrosis were markedly decreased in propolis-treated mice. Furthermore, AHR to acetylcholine was significantly abrogated in propolis-treated mice - These effects may be powerful weapons direct in the respiratory tract against the highly inflammatory SARS-CoV-2.

Regarding AMPs - the focus of the present study, especially propolis cationic AMPs -, as stated above, 
they are able to cross cell membranes and change their gene expression; so, they act on free-radicals through a much more complex antioxidant mechanism, not only through free radicals scavenging or simple inducing apoptosis. As known, excessive free radical generation, especially reactive oxygen species (ROS) that lead to oxidative stress in the biological system and generate the risk of chronic inflammation if ROS exceeds the antioxidant capacity, has been implicated in the pathogenesis and pathological conditions associated with several human inflammatory diseases. Therefore, in the normal resolution of inflammatory reactions, apoptosis is acknowledged to play a crucial role, while on the other hand, deregulation in the induction of apoptosis by enhanced ROS production could also result in excessive apoptosis identified in the pathogenesis of inflammatory diseases. Apparently, a careful balance must be maintained in this complex environment and antimicrobial peptides(AMPs) have been proposed as an excellent candidate capable of playing prominent roles in maintaining this balance, because they can be considered to possess both pro-inflammatory and anti-inflammatory properties, suggesting that they are key players in the inflammatory microenvironme nt .

In this way, it is speculated that propolis already amply discussedimmunomodulatory properties and possible synergy with the host immune system, which are sparsely already proven by several studies, are totally capable of preventing the cytokine storm and, more than just preventing it, may bring several benefits to the host respiratory system and whole organism. It is worth stressing again that the best effects are obtained when propolis is applied, keeping the cationic AMPs intact, direct to the infection site - in the case of SARS-CoV-2 in the respiratory tract through inhalation. AMPs are also able to modulate the host response and reduce the inflammatory response in sepsis. More recently, they have been found to interact directly with host's cells to modulate the inflammatory process and innate defenses. Sepsis is a recurrent condition during COVID-19 but the real cause is still not established, with a proposedhypothesis of viral sepsis. Therefore, propolis may certainly also prevent sepsis - even its phenolic compounds, as already quoted above, are able to block the NF-kappaB activation process and hence avoid sepsis .

\subsubsection{Possible Effects of the Cationic AMPs of Propolis on SARS-CoV-2 Secondary Consequences}

The main secondary consequence of SARS-CoV-2, as quoted, is that viral infections in the respiratory tract increase pneumococcal adherence to the local epithelium, facilitating bacterial infection . Coronavirus causes inflammatory damage in the lungs, preventing clearance of bacteria. As the present study demonstrated,propolis strong antibacterial effects are the most explored ones. Even in vivo, propolis showed antibacterial efficacy, despite studies focused only on the phenolic compounds and developed solely with peroral administration of the substance, not applying it directly to the infection site - which, as seen, may enhance the effects. Thus, now that propolis may be regarded as an AMPs-rich substance that should be applied direct to the infection/disease site, all the above quotedcatastrophic/brutal effects of cationic AMPs on bacterial biofilm and membranes may be applied, especially in the case of COVID-19 secondary bacterial infections. These effects may be stronger when propolis is applied directly to the infected lungs through inhalation, surely amplifying its antibacterial effects and hence avoiding pneumonia. It is worth to remember that propolis, althoughnot supposed to be resisted, if needed, can be safely used insynergy with conventional antibiotics. Also, propolis, if applied since the initial symptoms, may act as a strong prophylactic measure, helping the clearance and avoiding any bacterial growth inside the respiratory tract during the COVID-19. Therefore, the possible cationic AMPs contained in propolis are designed to have powerful selective/non-toxic antibacterial effects, which, in the case of SARS-CoV-2 secondary bacterial infections,may be the difference from life and death .

Moreover, many survivors of the 2003 outbreak of severe acute respiratory syndrome (SARS) developed residual pulmonary fibrosis, with increased severity seen in older patients. Autopsies of patients that died from SARS also showed fibrosis to varying extents. Pulmonary fibrosis can be occasionally seen as a consequence to several respiratory viral infections but is much more common after a SARS coronavirus (SARS-CoV) infection. SARS-CoV-2 has been reported to cause lung fibrosis. Beyond the trial already mentioned above, with inhaled propolis showing strong effect against fibrosis, a recent study with rats, 
using peroral administration, revealed that propolis diminished bleomycin induced lung fibrosis more effectively than prednisolone attributing it to its potent antioxidant and anti-inflammatory properties . Propolis possible cationic AMPs, with all the properties already quoted and also the strong wound healing effects - showing regenerative properties and accelerating the healing process -, mayavoid the occurrence of fibrosis and other sequelae.

Another effect already indicated of the cationic AMPs from propolis is the possible synergy with host's pulmonary surfactants. In SARS-CoV-2, this interaction may help toimprove breathing and oxygen saturation, preventing acute respiratory distress syndrome and lessening the need of artificial ventilation. Propolis, with all the mentioned effects in the treatment of COVID-19, even if mild, may surely buy the adaptive immune system more time to fight the disease and can possibly be effective against any respiratory diseases that might occur in consequence of SARS-CoV-2 infection. Propolis may also have cardioprotective effects - it is possible that cardiovascular damage can be a common consequence of COVID-19. The cardiovascular effects of propolis have been widely reported, although the underlying mechanisms have been poorly characterised. Results from research conducted tend to indicate that cardio-protective effects are the result of the antioxidant activity of propolis and its constituent compounds. As always, the focus of the studies of propolis cardio-protective effects was on the phenolic compounds; however, as shown, AMPs can have even more powerful antioxidant and immunomodulatory effects, and they are possibly the unknown underlying mechanisms. Hence propolis can possibly act as a cardioprotective substance, avoiding SARS-CoV-2 damage .

Beyond this, as a strong cationic amphipathic substance, propolis may "wash away" any other pathogens from the respiratory tract with no adverse effects or drug interactions, helping patients with comorbidities also due to the strong immunomodulatory effects. It is worth mentioning that propolis, given the physical mean of action , may be used effectively in any stage of COVID-19, not needing to be combined with any other drug(avoiding the risk associated with the highly toxic drugs that are being used). Lastly, propolis may have refreshing, revitalizing and soothing effects when applied directly to the respiratory tract, relieving the cough and pain caused by the viral infection .

As a little final observation, which will not be discussed in order to not deviate from the objective of the present study, it is speculated that propolis consumption (even through peroral administration) may somehow also contribute to possible herd immunity . For example, in Japan, which is known to consume huge amounts of propolis, despite a big population, COVID-19 cases so far are few and the number of deaths quite low in comparison to other countries in similar conditions, and it seems that it is not the first time that it happens in a pandemic . This is certainly something that should be investigated in deep.

\subsection{The Importance of Propolis Inhalation in the Treatment of COVID-19}

Propolis inhalation, as already seen above, may enhance the effects and allow the cationic AMPs to be delivered intact in the infection site, preserving the synergy of compounds and increasing the concentration (allowing the critical micelle concentration of the "detergent" by raising the peptide/lipid ratio, forming micelles that involve the pathogens/host's cells and related parts). So, propolisparenteral administration by means of inhalation may be the best existing way to use the substance in the treatment of COVID-19 - and possibly in several other diseases as other means of propolis parenteral administration are not available yet.

The cationic AMPs of propolis, as vastly seen, have physical/electrostatic action, so, direct application to the infection/disease site - in the case of respiratory infections/diseasesdirectly in the respiratory tract through inhalation - allows the direct contact of these AMPs with the affected tissues/invaders and their products and substances (the cationic AMPs do not previously interact/bind to any other organisms/agents that may inactivate them and prevent their action in the infection/disease site). This contactproduces direct effects/interaction in the site, with propolis positively charged AMPs physically binding to all the parts in the structure of the microbes/host's cells and their products/substances that 
have negative charges, potentially annihilating the invaders, and modulating the host immune system as well. Also, it is worth saying again that it is believed that direct administration through inhalation may be able to increase delivery and absorption of the active ingredients (cationic AMPs) throughout the entire organism, enhance systemic effects (immunomodulatory, furthering host homeostasis ), and diminish adverse effects through the digestive tract . In fact, as mentioned, the high surface area and high permeability of the lungs make them an ideal site for rapid systemic delivery of macromolecules and they are significantly permeable to many peptides and proteins - Again, it is worth stressing that the only two studies found specifically about propolis inhalation demonstrated promising results, showing significantreduction in the levels of several cytokines in bronchoalveolar lavage fluid and also high immunomodulatory capacity .

Inhalation, specifically in the case of COVID-19 treatments, has been explored in some studies and drug development projects as a promising tool - most of the desired/researched effects of these works are quite similar to those already observed when using propolis. In Germany, a study about a SARS-CoV-2 protease (3CLpro) inhibitor drug (13b) that is being tested stated that it is suitable to be administered through inhalation, furthering pharmacokinetic. Another study, already seen above, suggested the parenteral use of nebulised heparin against SARS-CoV-2 and stressed thatsuch a route of administration would not only be suitable for prophylaxis, but also for patients under mechanical ventilation. In the United States, because of COVID-19, there has been increased allocation of albuterol inhalers for the treatment of patients with or suspected to have COVID-19 infection . Even inhalation of nitric oxide is being tested in the treatment of COVID-19 because itappears to have direct antiviral effects . Pulmotecinhaled immunostimulant drug in development is also being tested against SARS-CoV-2; it is called PUL-042 and is made up of toll-like receptor ligands; it is supposed to stimulate the immune system in the lungs, protecting against a wide range of pathogens. Two other companies, Neurimmune and Ethris, joined to research, develop and produce an inhaled therapy for COVID-19using mRNA encoded neutralizing anti-SARS-CoV-2 antibodies; they claimed that local delivery in the lung may provide significant therapeutic benefits, rapidly achieving effective pulmonary antibody concentrations. Particularly in this last research and development of anti-SARSCoV-2 antibodies, propolis cationic AMPs may be a more powerful alternative. The mechanism of AMPs issimilar to the one of antibodies; they are innate defense peptides (may be compatible and may perfectly communicate with host's immune system) that are ready to be delivered in high amounts in the respiratory tract and fight this devastating disease.

Regarding propolis inhalation procedure in COVID-19, considering that there are no studies about the best manner or device ideal particle size to apply propolis and there are no propolis pMDIs (pressured metered dose inhalers) or DPI (dry powder inhalers), the substance must be inhaled through traditional nebulizers (pneumatic or ultrasonic), even in ICU patients. The basic procedure was already exposed above and the best one, especially in healthcare settings, should be evaluated by healthcare professionals according to each specific situation. Although there are some speculations that inhalation through nebulizers may contribute to patient infection by other pathogens and help to spread SARS-CoV-2, no evidence was found. There is a possibility that nebulizer therapy in patients with COVID-19 infection can transmit potentially viable coronavirus to susceptible bystander hosts . Because of this, in the United States, for example, as mentioned above about the use of inhaled albuterol in the treatment of COVID19 , there has been an increased allocation of albuterol inhalers for patients with or suspected to have COVID-19 infection due to concerns that nebulizers may help spread the virus in the air in hospitals . On the other hand, in a healthcare setting, a study inpatients that received nebulised medication administration recovered minimal viable bacteria from generated aerosols, mostly common environmental organisms. The study suggested that some of the procedures considered to be aerosol-generating may pose little infection risk to healthcare personnel. Anyway, given the high infectivity of SARS-CoV-2, some measures may avoid the possible transmission through nebulizer utilization. In a domestic setting, the isolation of the patient in a closed room with natural ventilation may be enough for a safe administration of inhaled propolis. In healthcare settings, besides all the safety 
procedures already adopted, carefully controlling the use and exposure to any respiratory assist devices (high-flow oxygen masks, nebulizers) by only allowing their use in designated, containment areas or rooms may be enough to avoid contamination. Another idea is the use of a cloth or some other tissue over the nebulizer mask (nozzle), similar to a face mask,to trap and avoid the spread of the aerosols . It is believed that, given the strong antimicrobial mechanisms of propolis, its nebulization may be safe as it may be able to neutralize, by physical action, any pathogen that may be exhaled or even inhaled by patients .

Therefore, propolis parenteral administration through inhalation, as said, may be the best existing way to use the substance in any stage of COVID-19 treatment, withcomprehensive effects that seem superior to any drug ever tested or produced. It is never enough to repeat that propolis inhalation may be used as a powerful prophylactic measure, especially by health professionals , turning their lungs into a "sterile bee hive" and hence avoiding any sort of contamination . As can be seen, thetreatment may be easy, allowing most patients to be treated at home with a possible high rate of success and minimal risk of intoxication and side effects. Also, given the inexistence of sufficient studies about propolis inhalation and the devastating nature of SARS-CoV-2, the combination of inhalation with propolis peroral administration must be advised .

\subsection{Possible Disadvantages of the Use of Propolis in the Treatment of COVID-19}

Propolis, as extensively demonstrated, despite some isolated cases of allergy, seems to have no adverse effects or any other observed disadvantages when used in the treatment of several infections/diseases. The only possible drawback foreseen is that, as a natural product,the amount produced throughout the world may not be enough to treat all COVID-19 patients. The good news is that countries in development are the biggest producers. As these countries do not have a solid health structure, they may immediately benefit of this possible new treatment. It is difficult to find official data about the production of propolis and its trade, but it seems that world's biggest producers are China, Russia and Brazil . Brazil produces around 150 MT yearly and China seems to produce more than 300 MT per year. The amount produced may be enough to fight COVID-19 and the production, maybe in a period of four to six months - time required for other bee products -, can be increased, especially in tropical climate places where bees produce all year. Other measures such as preventing sales of propolis as a supplement and directing it to health professionals and severely ill patients may also help to overcome this problem . Besides this, advancing the studies on the extraction of the AMPs from propolis, ideal dosage and means of administration may be another manner to rationalize the application of the substance.

Another possible disadvantage is the fact that propolis extraction is not standardized and its focus is on the phenolic compounds - and not, as seen, on the active ingredients that are the cationic AMPs. However, as vastly exposed, the existing extracts, ethanolic or aqueous, regardless of the origin, may be able to provide strong electrostatic effects. The efficacy of the extracts may be easily tested in order to measure if they have a minimum desired surfactant/detergent property, and the tests can be carried out in a similar manner to the experiments developed in the present study (especially through breaking of the surface tension of water). Thus, for now, while new studies are not developed, if this surfactant/detergent property is satisfactorily present, regardless of the mean of presentation or origin, it is believed that the extract will have the potential to successfully fight microbes/diseases .

\subsection{Comparison of Propolis with some of the Drugs currently being tested against COVID-19}

Hundreds of studies and clinical trials are underway throughout the world at the moment trying to find a successful treatment for COVID-19. World Health Organization recently launched a large international trial called Solidarity to test four existing therapies. They are the closely related malaria drugschloroquine and hydroxychloroquine; the antiviral medicationremdesivir (originally developed to treat Ebola); the antiviral combination of lopinavir and ritonavir (used for HIV); and those two HIV drugs plus the antiinflammatory small protein interferon beta. A number of separate clinical trials of these medications 
are underway in several countries. In this way, despite the innumerous other drugs in test, to spare time, the focus of the comparison will be on these six most tested drugs and alsocorticosteroids - as it seems that they are being extensively used in the treatment of COVID-19, even without evidence of efficacy .

In order to better illustrate the comparison between propolis and the drugs quoted above, it was done in the table below, using three colors to stress the features (in some of the notorious/known facts cited about the drugs, specific references were not utilized).

\begin{tabular}{|c|c|c|c|c|}
\hline $\begin{array}{l}\text { Possible Effects } \\
\text { on SARS-CoV- } \\
2 / \text { COVID-19 }\end{array}$ & Propolis & $\begin{array}{l}\text { Chloroquine/ } \\
\text { Hydroxychlor. }\end{array}$ & Antivirals & $\begin{array}{l}\text { Anti- } \\
\text { inflammatories }\end{array}$ \\
\hline $\begin{array}{l}\text { Mechanism of } \\
\text { action }\end{array}$ & $\begin{array}{l}\text { Various possible } \\
\text { antiviral struc- } \\
\text { tural/electrostatic } \\
\text { mechanisms of } \\
\text { action through } \\
\text { cationic AMPs; acts } \\
\text { directly on the } \\
\text { infection site } \\
\text { (especially through } \\
\text { inhalation) with } \\
\text { propolis positively } \\
\text { charged AMPs } \\
\text { physically binding } \\
\text { to all parts in the } \\
\text { structure of the } \\
\text { viruses/host's cells } \\
\text { and their prod- } \\
\text { ucts/substances } \\
\text { that are negatively } \\
\text { charged, potentially } \\
\text { annihilating the } \\
\text { virus, and } \\
\text { modulating host's } \\
\text { immune system as } \\
\text { well }\end{array}$ & $\begin{array}{l}\text { Unclear antiviral } \\
\text { mechanism. It } \\
\text { is hypothesized to } \\
\text { work by changing } \\
\text { the pH required for } \\
\text { SARS-CoV-2 to } \\
\text { replicate. }\end{array}$ & $\begin{array}{l}\text { Remdesivir: viral } \\
\text { polymerase (RdRp) } \\
\text { inhibitor, targeting } \\
\text { the viral genome } \\
\text { replication process; } \\
\text { Lopinavir/Ritonavi } \\
\text { viral protease } \\
\text { inhibitors, blocking } \\
\text { an enzyme involved } \\
\text { in HIV viral } \\
\text { replication. }\end{array}$ & $\begin{array}{l}\text { Interferon-beta: } \\
\text { complex } \\
\text { anti-inflammatory } \\
\text { and } \\
\text { immunomodulatory } \\
\text { rmechanisms. } \\
\text { Antiviral } \\
\text { mechanism not well } \\
\text { understood. } \\
\text { Corticosteroids: } \\
\text { Known } \\
\text { anti-inflammatory } \\
\text { mechanism of } \\
\text { action. No antiviral } \\
\text { mechanism found, } \\
\text { with some evidence } \\
\text { of promotion of } \\
\text { viral rebound if used } \\
\text { in later stages of } \\
\text { COVID-19. }\end{array}$ \\
\hline Viral envelope & $\begin{array}{l}\text { Possible (research } \\
\text { needed) }\end{array}$ & Not known & Not known & Not known \\
\hline Viroporins & $\begin{array}{l}\text { Possible (research } \\
\text { needed) }\end{array}$ & Not known & Not known & Not known \\
\hline $\begin{array}{l}\text { Decrease of viral } \\
\text { replication rate }\end{array}$ & $\begin{array}{l}\text { Possible (research } \\
\text { needed, several } \\
\text { studies in vitro } \\
\text { demonstrated } \\
\text { decrease in } \\
\text { replication of } \\
\text { other viruses) }\end{array}$ & $\begin{array}{l}\text { Possible (in } \\
\text { research, } \\
\text { notorious fact } \\
\text { that the results } \\
\text { are } \\
\text { unencouraging) }\end{array}$ & $\begin{array}{l}\text { Possible (in } \\
\text { research) } \\
\text { (Remdesivir acts } \\
\text { by targeting the } \\
\text { viral genome } \\
\text { repli- } \\
\text { cation process and } \\
\text { Lopinavir/ritonavir } \\
\text { by blocking } \\
\text { enzymes not } \\
\text { known) }\end{array}$ & $\begin{array}{l}\text { Not known } \\
\text { (Interferon-beta } \\
\text { may have a not } \\
\text { well known } \\
\text { antiviral } \\
\text { mechanism when } \\
\text { combined with } \\
\text { other antivirals) }\end{array}$ \\
\hline
\end{tabular}




\begin{tabular}{|c|c|c|c|c|}
\hline $\begin{array}{l}\text { Possible Effects } \\
\text { on SARS-CoV- } \\
\text { 2/COVID-19 }\end{array}$ & Propolis & $\begin{array}{l}\text { Chloroquine/ } \\
\text { Hydroxychlor. }\end{array}$ & Antivirals & $\begin{array}{l}\text { Anti- } \\
\text { inflammatories }\end{array}$ \\
\hline 3CLpro enzyme & $\begin{array}{l}\text { Possible (research } \\
\text { needed) }\end{array}$ & Not known & Not known & Not known \\
\hline $\begin{array}{l}\text { 2'-O-MTase } \\
\text { enzyme }\end{array}$ & $\begin{array}{l}\text { Possible (research } \\
\text { needed) }\end{array}$ & Not known & Not known & Not known \\
\hline Host's HSPG & $\begin{array}{l}\text { Possible (research } \\
\text { needed) }\end{array}$ & Not known & Not known & Not known \\
\hline Host's Heparin & $\begin{array}{l}\text { Possible (research } \\
\text { needed) }\end{array}$ & Not known & Not known & Not known \\
\hline Host's ACE2 & $\begin{array}{l}\text { Possible (research } \\
\text { needed) }\end{array}$ & Not known & Not known & Not known \\
\hline $\begin{array}{l}\text { Cytokine storm, } \\
\text { sepsis, fibrosis } \\
\text { (anti-inflammatory/ } \\
\text { immunomodulatory } \\
\text { effects) }\end{array}$ & $\begin{array}{l}\text { Demonstrated (high } \\
\text { Immunomodulatory } \\
\text { effects) (propolis, } \\
\text { e.g., showed } \\
\text { similar results in } \\
\text { comparison to } \\
\text { dexamethasone } \\
\text { in the treatment } \\
\text { of murine asthma } \\
\text { ) }\end{array}$ & $\begin{array}{l}\text { May reduce } \\
\text { inflammation } \\
\text { through an } \\
\text { unknown } \\
\text { mechanism (but } \\
\text { may impair the } \\
\text { ability of the } \\
\text { immune system to } \\
\text { fight infections) (it } \\
\text { is highly toxic, } \\
\text { mainly to the heart) }\end{array}$ & $\begin{array}{l}\text { Not known (may } \\
\text { help if proven that } \\
\text { reduces viral } \\
\text { replication rate) }\end{array}$ & $\begin{array}{l}\text { May } \\
\text { reduce/modulate } \\
\text { inflammation (but } \\
\text { may impair the } \\
\text { ability of the } \\
\text { immune system to } \\
\text { fight infections) } \\
\text { (Interferon-beta } \\
\text { may present risk if } \\
\text { used in later stages } \\
\text { as it can increase } \\
\text { the inflammatory } \\
\text { response) }\end{array}$ \\
\hline $\begin{array}{l}\text { Antibacterial } \\
\text { (similar or } \\
\text { stronger than } \\
\text { conventional } \\
\text { antibiotics) (able } \\
\text { to help fighting } \\
\text { secondary } \\
\text { bacterial } \\
\text { infections) }\end{array}$ & $\begin{array}{l}\text { Demonstrated (by } \\
\text { various studies } \\
\text { quoted above and } \\
\text { vastly discussed) }\end{array}$ & $\begin{array}{l}\text { Not known (may } \\
\text { cause } \\
\text { vulnerability to } \\
\text { other bacterial or } \\
\text { viral infections by } \\
\text { suppressing the } \\
\text { immune system) } \\
\text { (It is known that } \\
\text { in some cases it is } \\
\text { being used in } \\
\text { combination w/ } \\
\text { azithromycin) }\end{array}$ & $\mathrm{N} / \mathrm{A}$ & $\begin{array}{l}\text { Weak } \\
\text { (Corticosteroids } \\
\text { do not appear to } \\
\text { have widespread } \\
\text { antibacterial } \\
\text { properties ) } \\
\text { Interferon-beta } \\
\text { may have } \\
\text { antimicrobial } \\
\text { properties }\end{array}$ \\
\hline $\begin{array}{l}\text { Synergy w/ other } \\
\text { antibiotics }\end{array}$ & Demonstrated & $\mathrm{N} / \mathrm{A}$ & $\mathrm{N} / \mathrm{A}$ & Not known \\
\hline Prophylactic use & $\begin{array}{l}\text { Possible (research } \\
\text { needed) }\end{array}$ & $\begin{array}{l}\text { Not known (in } \\
\text { research; } \\
\text { notorious fact } \\
\text { that the results } \\
\text { are } \\
\text { unencouraging) }\end{array}$ & Not known & Not known \\
\hline $\begin{array}{l}\text { Synergy with host } \\
\text { pulmonary } \\
\text { surfactants }\end{array}$ & $\begin{array}{l}\text { Possible (research } \\
\text { needed) }\end{array}$ & $\mathrm{N} / \mathrm{A}$ & $\mathrm{N} / \mathrm{A}$ & $\mathrm{N} / \mathrm{A}$ \\
\hline
\end{tabular}




\begin{tabular}{|c|c|c|c|c|}
\hline $\begin{array}{l}\text { Possible Effects } \\
\text { on SARS-CoV- } \\
2 / \text { COVID-19 }\end{array}$ & Propolis & $\begin{array}{l}\text { Chloroquine/ } \\
\text { Hydroxychlor. }\end{array}$ & Antivirals & $\begin{array}{l}\text { Anti- } \\
\text { inflammatories }\end{array}$ \\
\hline $\begin{array}{l}\text { Cardioprotective, } \\
\text { help w/ host's } \\
\text { comorbidities }\end{array}$ & $\begin{array}{l}\text { Demonstrated (high } \\
\text { Immunomodulatory } \\
\text { effects vastly } \\
\text { discussed above) }\end{array}$ & $\begin{array}{l}\text { Not known (may } \\
\text { present cardiac } \\
\text { toxicity) }\end{array}$ & Not known & $\begin{array}{l}\text { May } \\
\text { reduce/modulate } \\
\text { inflammation (but } \\
\text { it is a known fact } \\
\text { that both may be } \\
\text { harmful in case of } \\
\text { comorbidities) }\end{array}$ \\
\hline $\begin{array}{l}\text { Possibility of viral } \\
\text { resistance to the } \\
\text { substance }\end{array}$ & $\begin{array}{l}\text { Inexistent (if the } \\
\text { physical } \\
\text { mechanism is } \\
\text { definitely proven) }\end{array}$ & $\begin{array}{l}\text { Possible } \\
\text { (resistance of } \\
\text { the own } \\
\text { Plasmodium } \\
\text { falciparum was } \\
\text { already } \\
\text { documented }\end{array}$ & $\begin{array}{l}\text { Yes (as various } \\
\text { studies have } \\
\text { been } \\
\text { demonstrating) }\end{array}$ & $\mathrm{N} / \mathrm{A}$ \\
\hline $\begin{array}{l}\text { Administration in } \\
\text { any stage of the } \\
\text { disease }\end{array}$ & $\begin{array}{l}\text { Possible (research } \\
\text { needed) }\end{array}$ & $\begin{array}{l}\text { Not known (in } \\
\text { research) }\end{array}$ & $\begin{array}{l}\text { Not Known (in } \\
\text { research) }\end{array}$ & $\begin{array}{l}\text { Not Known (in } \\
\text { research) }\end{array}$ \\
\hline $\begin{array}{l}\text { Use through } \\
\text { inhalation, } \\
\text { enhancing effects }\end{array}$ & $\begin{array}{l}\text { Demonstrated } \\
\text { (although by a few } \\
\text { studies; } \\
\text { demonstrating no } \\
\text { adverse but im- } \\
\text { munomodulatory } \\
\text { effects) }\end{array}$ & Not known & $\begin{array}{l}\text { Not known } \\
\text { (remdesivir is } \\
\text { administered } \\
\text { through IV } \\
\text { infusion) }\end{array}$ & $\begin{array}{l}\text { Demonstrated (it } \\
\text { is a known fact } \\
\text { that more } \\
\text { research is } \\
\text { needed, especially } \\
\text { for interferon } \\
\text { safety and specific } \\
\text { effects) }\end{array}$ \\
\hline $\begin{array}{l}\text { General } \\
\text { detergent-like } \\
\text { effects }\end{array}$ & $\begin{array}{l}\text { Demonstrated in } \\
\text { the present study }\end{array}$ & $\mathrm{N} / \mathrm{A}$ & $\mathrm{N} / \mathrm{A}$ & $\mathrm{N} / \mathrm{A}$ \\
\hline $\begin{array}{l}\text { Similar or more } \\
\text { powerful effects } \\
\text { than other } \\
\text { antivirals } \\
\text { (oseltamivir, } \\
\text { acyclovir, } \\
\text { ribavirin) }\end{array}$ & Demonstrated & $\mathrm{N} / \mathrm{A}$ & Not known & $\mathrm{N} / \mathrm{A}$ \\
\hline $\begin{array}{l}\text { Similar effects in } \\
\text { comparison with } \\
\text { chloroquine } \\
\text { (anti-plasmodial) }\end{array}$ & Demonstrated & $\mathrm{N} / \mathrm{A}$ & $\mathrm{N} / \mathrm{A}$ & $\mathrm{N} / \mathrm{A}$ \\
\hline
\end{tabular}




\begin{tabular}{|c|c|c|c|c|}
\hline $\begin{array}{l}\text { Possible Effects } \\
\text { on SARS-CoV- } \\
2 / \text { COVID-19 }\end{array}$ & Propolis & $\begin{array}{l}\text { Chloroquine/ } \\
\text { Hydroxychlor. }\end{array}$ & Antivirals & $\begin{array}{l}\text { Anti- } \\
\text { inflammatories }\end{array}$ \\
\hline Adverse Effects & $\begin{array}{l}\text { None (small } \\
\text { probability of } \\
\text { allergy) }\end{array}$ & $\begin{array}{l}\text { Many (notorious } \\
\text { fact, due to the } \\
\text { high toxicity; } \\
\text { cause the increase } \\
\text { in inflammation } \\
\text { and has immuno- } \\
\text { suppressive } \\
\text { effects) }\end{array}$ & $\begin{array}{l}\text { Many (besides } \\
\text { viral ones, } \\
\text { notoriously } \\
\text { inhibits body } \\
\text { enzymes causing } \\
\text { harm; remdesivir } \\
\text { is still } \\
\text { experimental, so, } \\
\text { adverse effects are } \\
\text { not deeply } \\
\text { studied) }\end{array}$ & $\begin{array}{l}\text { Many (vastly } \\
\text { documented by } \\
\text { science) }\end{array}$ \\
\hline $\begin{array}{l}\text { Possible harmful } \\
\text { interaction with } \\
\text { other drugs }\end{array}$ & $\begin{array}{l}\text { None so far (more } \\
\text { research needed; } \\
\text { possible synergy) }\end{array}$ & $\begin{array}{l}\text { Many (vastly } \\
\text { documented by } \\
\text { science) }\end{array}$ & $\begin{array}{l}\text { Many (notorious } \\
\text { because of their } \\
\text { mechanism of } \\
\text { action, affecting } \\
\text { body processes } \\
\text { and organs) }\end{array}$ & $\begin{array}{l}\text { Many (vastly } \\
\text { documented by } \\
\text { science) }\end{array}$ \\
\hline $\begin{array}{l}\text { Already being } \\
\text { produced in large } \\
\text { scale }\end{array}$ & $\begin{array}{l}\text { Possibly } \\
\text { (production can be } \\
\text { increased in a } \\
\text { relatively small time } \\
\text { as stated above) }\end{array}$ & $\begin{array}{l}\text { Yes (production is } \\
\text { being increased } \\
\text { because of the } \\
\text { possible use against } \\
\text { SARS-CoV-2) }\end{array}$ & $\begin{array}{l}\text { No (Remdesivir is } \\
\text { still experimental } \\
\text { and it is known that } \\
\text { the production } \\
\text { possibly will be } \\
\text { increased if the } \\
\text { effects are proven) } \\
\text { (Lopinavir/Ritonavir: } \\
\text { used in HIV } \\
\text { treatment, so the } \\
\text { production may } \\
\text { be increased, but } \\
\text { it is a } \text { known fact } \\
\text { that it is still } \\
\text { small) }\end{array}$ & $\begin{array}{l}\text { Yes (as traditional } \\
\text { drugs, it is known } \\
\text { that the production } \\
\text { can be increased } \\
\text { rapidly) }\end{array}$ \\
\hline
\end{tabular}

The comparison, although many effects have yet to be proven, can be summarized by an illustrative metaphor. In host's fight against SARS-CoV-2 there are basically two existing strategies to try to help host's own troops in this unfair war. The first one is to depend on host's own army and use one of the existing drugs in test quoted above that are able to possibly affect one little supply used by the enemy invader (antivirals) or create a little unfavourable situation on its grounds (chloroquine) . The second strategy is to use propolis, possibly bringing in and implanting a strong allied army to help host's troops in the battle. As an observation, one other strategy/effect in the quoted drugs in test that is not even going to be detailed, given the possible lack of sense and the strong possibility of rapid viral takeover, is using drugs (such asInterferon-beta and Corticosteroids ) that slow the response and diminish/weaken host's virus fighting troops to try to avoid the collapse of the nation .

Regarding the first strategy cited, in the case of drugs that affect supplies used by the enemy (antivirals), it is worth saying that this sort of supplies are also used by host's soldiers, civilians , communities, societies, and for whole nation functions, which may put them all in a dangerous shortage as well. Moreover, the other drug effect of creating anunfavorable situation for the virus 
(chloroquine, which may also decrease the response of host's troops) may be also toxic for host's soldiers, civilians, communities and societies, and the whole nation. Thus, the lack of supply or the unfavourable situation generated, bothmay weaken host's virus fighting troops, affecting their capacity to fight the invader, and also put the whole nation balance (homeostasis) in risk of collapse because of the shortage and/or adverse situation generated throughout the whole body . These lack of supply or unfavourable situation effects of the first strategy may also beeasily overcome by the enemy that may be able to rapidly change the supplies used or rapidly adapt to the unfavorable grounds - and the enemy is known to be very fast. In these last scenarios that may harm the host, the enemy invader may take advantage of host's defense weaknesses and nation imminent collapse, and reproduce in huge amounts, finally conquering the entire organism .

The second suggested strategy to fight SARS-CoV-2 is by usingpropolis and the cationic AMPs that it possibly contains. That is, to implant inside the host a complete high-tech army with abroad spectrum of different function soldiers - there are soldiers that can attack the enemy armor and body directly; others can cut the enemy supplies or prevent them to take/use civilians by blocking the road; they even can get into host's soldiers/civilians "mind" and reprogram it to "commit suicide" or function correctly and become an ally and help. Through these direct actions in the battlefield, these allied complete troops may help to neutralize the actions of the enemy invaders, preventing them to reproduce in huge amounts and domain the entire organism, potentially annihilating the invaders and modulating the defenses. In addition, given the similar language and mechanism of action with host's troops, the implanted ones can perfectly communicate with the local soldiers and work in synergy with them. This connection is also valid for communications between the implanted army and host's civilians. The messages sent indirectly helps in the battle by reprogramming civilians to resist the invaders, modulating their response and hence avoiding threats to the balance (homeostasis) and eventual collapse of the whole nation.

As can be seen, it is as simple as it looks, the first and most important lines of defense, which are the AMPs, are strongly positively charged and keep the "electrostatic patrol" against the negatively charged invaders; also sending correct physical/chemical messages to host's cells . This is a high-tech and natural selective way that perfectly distinguishes the enemy from health cells, also crossing host cell membranes and acting in the DNA level to help fight the infection. Thus, maybe now the possibility of "implanting" a powerful cationic electrostatic complete army in any amount needed to fight infections with ample success (even better than only "cruise missiles") is closer than ever imagined. In this way, it is suggested and emphasized that further scientific trials on propolis should be developed urgently, especially in humans given the possible selectivity and absence of toxicity of the substance. If all properties and health benefits of the cationic AMPs from propolis are definitely proven, these special peptides are supposed to achieve $100 \%$ of efficacy in $100 \%$ of cases and may be the most powerful weapon ever found, not only against SARS-Cov-2, but against all respiratory tract infections - and, as seen, possibly against a broad range of other diseases.

\section{Anecdotal Evidence of Propolis Use in the Treatment of Possible COVID-19}

Given the present planetary emergency, as a starting point,although no statistical significance and very low scientific value, in order to encourage further scientific clinical trials and provide some practical hints, the treatment with propolis of two individuals suspected of having COVID-19 will be summarized like a case study.

\subsection{The Subjects and Symptomatology}

The two subjects were a 33 year old woman and a 40 year old man, without any allergies or comorbidities and not under any medication. Unfortunately in Brazil tests for COVID-19 were scarce and only applied to hospitalized patients. Thus, the possible infection was diagnosed only by symptomatology, which is described in the table below. 


\begin{tabular}{ll}
\hline Subject & Symptoms \\
\hline Woman $(56 \mathrm{~kg} 1.70 \mathrm{~m})$ & Traveled to COVID-19 suspect location; dry cough (no nasal congestion or runny nose); pleuritic ch \\
Man $(80 \mathrm{Kg}, 1.83 \mathrm{~m})$ & Traveled to COVID-19 suspect location; dry cough (no nasal congestion or runny nose); pleuritic ch \\
\hline
\end{tabular}

\subsection{Treatment}

Both subjects were treated with $11 \%$ propolis ethanolic extract (PEE)11Apis Flora Brazilian Green Propolis ethanolic extract. $11 \% \mathrm{w} / \mathrm{v}$ through oral administration and inhalation. The treatment started in the acute phase, i.e., when the fever started. No drugs or other natural substances were administered. The PEE was administered equally in both subjects for 14 days, as described below.

Acute phase (first 5 days; attack dosage):

Oral administration

Approx. $2 \mathrm{ml} \mathrm{(}$

Inhalation (domestic inhaler device/nebulizer w/ 50 PSI and 0.4 ml/min nebulization rate) 15 drops in $15 \mathrm{r}$ Total dosage

After the acute phase, maintenance (9 days):

Oral administration

Inhalation (domestic inhaler device $\mathrm{w} / 50$ PSI and $0.4 \mathrm{ml} / \mathrm{min}$ nebulization rate) Total dosage
Approx. $1.5 \mathrm{ml}(30$ drops o

15 drops in $15 \mathrm{ml}$ of Sterile $742 \mathrm{mg}$ per day

\subsection{Results}

The most important part of the treatment was during the acute phase, in which all the progress was made. The second phase was solely maintenance. So, only the acute phase treatment of both subjects will be described in the table below, remembering that the treatments started when the high fever suddenly started and, besides propolis extract, no other natural substances or drugs were used.

\begin{tabular}{lll}
\hline Day & Woman & Man \\
\hline 1 & Dry cough: strong (started to & Dry cough: strong Pleuritic \\
get wet) Pleuritic chest pain: & chest pain: strong Diarrhea: \\
strong Dizziness: strong Severe & strong Tiredness: strong \\
& tiredness: strong Breathing & Breathing difficulty: moderate \\
& difficulty: strong (seemed to & Elevated heart rate: present \\
& ameliorate after inhalation & Fever: high; started lowering in \\
& sessions) Tachycardia: present & just one hour (below $37.2^{\circ} \mathrm{C}$ ) and \\
& Fever: high; was controlled and & in approximately 12 hours it \\
& lowered to $37.2^{\circ} \mathrm{C}$ in less than 2 & was totally cleared
\end{tabular}

hours, varying below this

temperature for a time, and in

approximately 18 hours it was

totally cleared (maybe a higher

attack dosage with more

inhalation sessions could

have been more effective in

the fever clearance) 


\begin{tabular}{|c|c|c|}
\hline Day & Woman & Man \\
\hline 2 & $\begin{array}{l}\text { Dry cough: strong (wet, w/ a lot } \\
\text { of secretion) Pleuritic chest } \\
\text { pain: strong Dizziness: absent } \\
\text { Severe tiredness: weak } \\
\text { Breathing difficulty: moderate } \\
\text { Tachycardia: present Fever: } \\
\text { absent }\end{array}$ & $\begin{array}{l}\text { Dry cough: strong (started to } \\
\text { get wet) Pleuritic chest pain: } \\
\text { moderate Diarrhea: strong } \\
\text { Tiredness: moderate Breathing } \\
\text { difficulty: weak Elevated heart } \\
\text { rate: present Fever: absent }\end{array}$ \\
\hline 3 & $\begin{array}{l}\text { Dry cough: strong (wet, w/ a lot } \\
\text { of secretion) Pleuritic chest } \\
\text { pain: moderate Dizziness: } \\
\text { absent Severe tiredness: absent } \\
\text { Breathing difficulty: weak } \\
\text { Tachycardia: absent Fever: } \\
\text { absent }\end{array}$ & $\begin{array}{l}\text { Dry cough: strong (wet, w/ a lot } \\
\text { of secretion) Pleuritic chest } \\
\text { pain: moderate Diarrhea: } \\
\text { strong Tiredness: weak } \\
\text { Breathing difficulty: absent } \\
\text { Elevated heart rate: absent } \\
\text { Fever: absent }\end{array}$ \\
\hline 4 & $\begin{array}{l}\text { Dry cough: strong (wet, w/ a lot } \\
\text { of secretion) Pleuritic chest } \\
\text { pain: weak Dizziness: absent } \\
\text { Severe tiredness: absent } \\
\text { Breathing difficulty: weak } \\
\text { Tachycardia: absent Fever: } \\
\text { absent }\end{array}$ & $\begin{array}{l}\text { Dry cough: strong (wet, w/ a lot } \\
\text { of secretion) Pleuritic chest } \\
\text { pain: weak Diarrhea: moderate } \\
\text { Tiredness: absent Breathing } \\
\text { difficulty: absent Elevated } \\
\text { heart rate: absent Fever: } \\
\text { absent }\end{array}$ \\
\hline 5 & $\begin{array}{l}\text { Dry cough: moderate wet } \\
\text { Pleuritic chest pain: absent } \\
\text { Dizziness: absent Severe } \\
\text { tiredness: absent Breathing } \\
\text { difficulty: absent Tachycardia: } \\
\text { absent Fever: absent }\end{array}$ & $\begin{array}{l}\text { Dry cough: strong (wet, w/ a lot } \\
\text { of secretion) Pleuritic chest } \\
\text { pain: weak Diarrhea: moderate } \\
\text { Tiredness: absent Breathing } \\
\text { difficulty: absent Elevated } \\
\text { heart rate: absent Fever: } \\
\text { absent }\end{array}$ \\
\hline
\end{tabular}

As indicated, the most notorious effects of the treatment were therapid fever clearance and the fast relief in the overall symptoms. Both subjects reported refreshing effects during and after propolis inhalation and that it also seemed to help with the respiratory symptoms, helping breathing easily and the secretions to come out. As adverse effects, both subjects (one was already with diarrhea) only reported some gut issues that resolved in approximately 10 days after propolis use was totally suspended.

\subsection{Discussion}

Notwithstanding the fact that it was not possible to present laboratory tests as evidence that the reported infections were caused by SARS-CoV-2, at least, the symptoms presented allow to assume that a strong respiratory tract infection was present in both subjects. The symptoms, especially the fever, in which the clearance was quite high, were resolved quickly and in a similar manner in both subjects. Propolis utilization provided prompt relief of the symptoms without the use of any other concomitant therapeutic agent. The treatments could have started earlier, but the acute phase was chosen in order to better measure propolis effectiveness against strong infections . Maybe a higher initial dosage, with more inhalation sessions, could have been more effective in the prompt fever clearance.

The findings described suggest that propolis may be a promising effective and powerful tool against respiratory tract infections and hence against SARS-CoV-2 . It is worth mentioning that, in this relatively long treatment, almost no adverse effects were observed. This is also one more indication that propolis may be safely used in larger doses than commonly used as a supplement and is also safe to be used through inhalation. The effective dosage used to achieve the results showed 
were still very low $(\mathbf{1}, \mathbf{2 1 0} \mathrm{mg}$ per day in the acute phase) comparing to the dosage discussed above that may be safely elevated up to $\mathbf{1 4 , 0 0 0 ~} \mathbf{m g}(\mathbf{1 4 g})$ daily for an adult weighting $70 \mathrm{~kg}$. This allows a really safe dosage range for treatment, especially in more severe cases . The whole treatment used $12,728 \mathrm{mg}$ or $\mathbf{1 2 . 7 3} \mathbf{g}$ for each subject. If this amount is the sufficient dosage for most cases, in a simplified calculation, it means that the amount of raw propolis needed for each treatment is about $\mathbf{3 4 . 7 2}$ $\mathrm{g}$ - remembering that the extract production uses $30 \%$ of raw dry propolis, ending up with approximately $11 \% \mathrm{w} / \mathrm{v}$ of dry propolis in the final product. As mentioned above, for example, the production in Brazil is around $150 \mathrm{MT}$ yearly, which means that it may be possible to treat a total of $4,320,000$ infected people in a year; or 360,000 monthly . This may be more than enough to tackle SARS-CoV-2 outbreak, and the production may be increased in a relatively short time.

Lastly, as seen above, perhaps inhalation is the best mean for propolis administration and may be the only one to be used in the treatment of respiratory tract infections (also lowering the overall necessary dosage); but, considering the inexistence of sufficient studies about propolis inhalation and the devastating nature of SARS-CoV-2, thecombination of inhalation with propolis peroral administration must be advised - Propolis may bring the possibilities of prophylactic/early stage intervention, fast resolution of symptoms and home treatment, which are truly desirable features in the treatment of COVID-19 as they may lessen hospitalization time and help to relief the burden in health systems .

\section{Final Conclusion}

Despite the necessity of further studies, all the arguments and evidence presented seem remarkable and it cannot be rejected that propolis has a large amount of cationic AMPs, which produce strong effects. This 120 million-year-old high-tech electrostatic immune system that can be "borrowed" from bees and safely "implanted" in humans, possibly bringing together the expected effects of various therapeutic agents, may be now ready to be vastly studied and used against a wide range of infections/diseases. Given the possible "yin and yang" electrostatic mechanism that seems to rule most infections/diseases, maybe the code is already cracked and the "cruise missiles" principle envisioned forty years ago is more than viable. Physics may not only outsmart viruses, but all microbes/diseases. If this promising "nature versus nature" electrostatic system is definitely proven, propolis may become the medicine of the future. Thus, it is suggested that the hypothesis of the existence of a large amount of cationic AMPs in propolis and all implications on human health and on the production, extraction and administration of the substance, should be thoroughly explored by science.

Perhaps all these findings on propolis came at an opportune time and the substance may be ready to be used now as a weapon against SARS-CoV-2, mainly by means of inhalation. Propolis, as vastly demonstrated throughout this study, has already shown more than sufficient preclinical proof and safety to be tested in humans and may be a viable and promising treatment alternative against SARS-CoV-2, especially in comparison with the traditional drugs that are being tested at the moment. Therefore, in the current pandemic caused by this devastating virus, considering the possible potential to save thousands of lives, it is suggested that further studies on propolis should start urgently, primarily clinical trials on its possible efficacy in the treatment of COVID-19 .

\section{Acknowledgement}

The author would like to thank his wife, Marcela S. P. Burger, for all the assistance and help. Also, the author would like to thank the scientists, who, in their studies, pointed to fruitful directions: J. Bryan, P. Redden and C. Traba from the Department of Chemistry of Saint Peter's University in Jersey City, USA; Katrina Brudzynski and Calvin Sjaarda from the Drug Discovery and Development Department, Bee-Biomedicals Inc., St. Catharines, Ontario, Canada; and Rachel L. Vannette, Abbas Mohamed and Brian R. Johnson from the Department of Entomology and Nematology, University of California, USA.

\section{Declaration of Competing Interest}

The author declares no competing interest. 


\section{Funding}

This study was funded with author's personal resources.

\section{Ethical Approval}

Not required - anecdotal evidence of the treatments was obtained in the author's family and domestic setting, without the use of any drugs.

\section{Consent for publications}

All participants consented with the release of the objective data utilized in the study. No specific personal data were released.

\section{Disclosure}

All content and information in the present study are experimental and intended for further scientific studies and evaluation. Therefore, the author cannot be held responsible for any unexpected reactions or damage that the eventual use of propolis - a natural and freely sold substance, generally regarded as a dietary supplement - may cause on those who decide to utilize it. Propolis can cause severe reactions and allergies if misused or utilized by the wrong person. Health professional supervision is advised for propolis administration, especially for those with allergies, comorbidities and/or under medication.

\section{Copyright}

Copyright protected by Avctoris certificate $\mathrm{n}^{\mathrm{O}}$ 79fca8fc55ee7e3682bc16bc3a6b. This is an open-access article. Unrestricted use, distribution, and reproduction are allowed in any medium, provided the original author and source are credited.

\section{References}

1. The Editors of Encyclopaedia Britannica. Honeybee, Insect. Apini, honey bee.(2020) https://www.britannica.com/animal/honeybee.Encyclopaedia Britannica. Mar 24, 2020.

2. Wagh, V.D. Propolis: A Wonder Bees Product and Its Pharmacological Potentials. Adv Pharmacol Sci. Dec 9, 2013, Vol. 308249.

3. Kuropatnicki, A.K.; Szliszka, E.; Krol, W. Historical Aspects of Propolis Research in Modern Times. Evid Based Complement Alternat Med: 964149. Apr 28, 2013.

4. Finstrom, M.S.; Spivak, M. Propolis and bee health: the natural history and significance of resin use by honey bees. Apidologie.2010, Vol. 41, pp. 295-311.

5. Finstrom, M.S.; et al. Propolis Counteracts Some Threats to Honey Bee Health. Insects. 2017 Jun; 8(2): 46.doi: 10.3390/insects8020046. Cross ref.

6. Finstrom, M.D.S.; Spivak, M. Increased Resin Collection after Parasite Challenge: A Case of SelfMedication in Honey Bees? PLoS One. 2012; 7(3): e34601. Mar 29, 2012.

7. Borba, R.S.; Spivak, M. Propolis envelope in Apis mellifera colonies supports honey bees against the pathogen, Paenibacillus larvae.Scientific Reports. 2017, Vol. 7.

8. Bryan, J.; Redden, P.; Traba, C. The mechanism of action of Russian propolis ethanol extracts against two antibiotic-resistant biofilm-forming bacteria. Letters in Applied Microbiology 62. pgs 192-198. Free Access. ISSN 0266-8254. Nov 25, 2015.

9. Braakhuis, A. Evidence on the Health Benefits of Supplemental Propolis. Faculty of Medical Health Science, The University of Auckland, Auckland 1010, New Zealand. Nov 8, 2019. 
10. Takemura, T.; et al. 3,4-Dicaffeoylquinic Acid, a Major Constituent of Brazilian Propolis, Increases TRAIL Expression and Extends the Lifetimes of Mice Infected with the Influenza A Virus.Evidence-based Complementary and Alternative Medicine : Ecam. Aug 24, 2011.

11. Shimizu, T.; et al. Anti-influenza virus activity of propolis in vitro and its efficacy against influenza infection in mice.Antivir Chem Chemother. 2008.

12. Schnitzler, P.; et al. Antiviral activity and mode of action of propolis extracts and selected compounds. Phytother Res. April 24, 2010.

13. Yildirim, A.; et al. Antiviral Activity of Hatay Propolis Against Replication of Herpes Simplex Virus Type 1 and Type 2. Med Sci Monit. Feb 9, 2016.

14. Kwon, M.J.; et al. Antiviral effects and possible mechanisms of action of constituents from Brazilian propolis and related compounds. Journal of Apicultural Research (on line). Dec 52019.

15. Geker, G.; et al. Anti-HIV-1 activity of propolis in CD4(+) lymphocyte and microglial cell cultures. $J$ Ethnopharmacol. Nov 14, 2005.

16. Harish, Z.; et al. Suppression of HIV-1 replication by propolis and its immunoregulatory effect. Drugs Exp Clin Res. 1997.

17. Coelho, G.R.; et al. Antiviral effects of Scaptotrigona postica propolis and their fractions. BMC Proc 8, P63. 2014.

18. Przybyłek, I.; Karpiński, T.M. Antibacterial Properties of Propolis.Molecules. Jun, 2019.

19. Afrouzan, H.; Zakeri, S.; et al. Anti-Plasmodial Assessment of Four Different Iranian Propolis Extracts. Arch Iran Med.;. 2017, pp. 20(5): 270 - 281.

20. Olayemi, K. I. Therapeutic Potentials of Nigerian Insect-propolis Against the Malarial Parasite, Plasmodium berghei (Haemosporida: Plasmodidae). American Journal of Drug Discovery and Development.2014, pp. Volume 4 (4): 241-247.

21. Santoro, M. G.; Rossi, A.; Amici, C. NF- $\chi \mathrm{B}$ and virus infection: who controls whom. The Embo Journal Vol. 22 $n^{\circ}$ 11, pp. 2552-2560; Jun 1, 2003.

22. Koo, H.; et al. Effect of apis mellifera propolis from two Brazilian regions on caries development in desalivated rats. Caries Res.1999, pp. 33:393-400.

23. Orsi, R.O.; et al. Immunomodulatory action of propolis on macrophage activation. J. Venom. Anim. Toxins [online], vol.6, n.2.2000, pp. 205-219.

24. Pribul, P.K.; et al. Alveolar Macrophages Are a Major Determinant of Early Responses to Viral Lung Infection but Do Not Influence Subsequent Disease Development. JOURNAL OF VIROLOGY. May, 2008, pp. 4441-4448.

25. Tao, Y.; et al. The immunological enhancement activity of propolis flavonoids liposome in vitro and in vivo. Evid Based Complement Alternat Med. 2014, p. 483513.

26. Scheller, S.; et al. The ability of ethanol extract of propolis to stimulate plaque formation in immunized mouse spleen cells.Pharmacol Res Commun 20. 1988, pp. 323-8.

27. Al-Hariri, M. Immune's-boosting agent: Immunomodulation potentials of propolis. J Family Community Med 26(1). Jan-Apr, 2019, pp. 57-60.

28. Kimoto, T.; et al. Apoptosis and suppression of tumor growth by artepillin C extracted from Brazilian propolis. Cancer Detect Prev 22(6). 1998, pp. 506-15.

29. Alberts, B.; et al. Molecular Biology of the Cell. 4th edition. New York : Garland Science, 2002. 
30. Schimdt, M.E.; Varga, S. M. The CD8 T Cell Response to Respiratory Virus Infections. Front. Immunol. April 92018.

31. Liting, Z.; et al. Brazilian Green Propolis Improves Antioxidant Function in Patients with Type 2 Diabetes Mellitus. International Journal of Environmental Research and Public Health. May 13, 2016.

32. Samadi, N.; et al. Effects of bee propolis supplementation on glycemic control, lipid profile and insulin resistance indices in patients with type 2 diabetes: a randomized, double-blind clinical trial. $J$ Integr Med.15(2): Mar 2017, pp. 124-134.

33. Lopes, A.A.; et al. Antioxidant action of propolis on mouse lungs exposed to short-term cigarette smoke. Bioorganic \&3 Medicinal Chemistry. 24, Dec 15, 2013, Vol. 21, pp. 7570-7577.

34. Farias, J.H.C.; et al. Effects of Stingless Bee Propolis on Experimental Asthma. Evid Based Complement Alternat Med: 951478.Apr 12014.

35. Cohen, H.A.; et al. Effectiveness of an herbal preparation containing echinacea, propolis, and vitamin $\mathrm{C}$ in preventing respiratory tract infections in children: a randomized, double-blind, placebo-controlled, multicenter study. Arch Pediatr Adolesc Med.158(3). Mar, 2004, pp. 217-21.

36. Di Pierro, F.; Zanvit, A.; Colombo, M. Role of a proprietary propolis-based product on the wait-and-see approach in acute otitis media and in preventing evolution to tracheitis, bronchitis, or rhinosinusitis from nonstreptococcal pharyngitis. Int J Gen Med. eCollection 2016. 2016, Vol. 11, pp. 409-414.

37. Li, Y. J.; et al. Acute renal failure induced by a Brazilian variety of propolis. Am. J. Kidney Dis. 46. 2005, pp. e125-e129.

38. Basavaiah, N.D.; Suryakanth, D.B. Propolis and allergic reactions.J Pharm Bioallied Sci. 4, Oct-Dec 2012, Vol. 4, p. 345.

39. Fernandes, A.J.; et al. Propolis: anti-Staphylococcus aureus activity and synergism with antimicrobial drugs. Mem. Inst. Oswaldo Cruz. 5, Aug 2005, Vol. 100.

40. Kalia, P.; Kuma, N.R.; Harjai, K. Studies on the therapeutic effect of propolis along with standard antibacterial drug in Salmonella enterica serovar Typhimurium infected BALB/c mice. BMC Complement Altern Med. 2016; 16: 485. 485, Nov 25, 2016, Vol. 16.

41. Ambi, A.; et al. Are Russian propolis ethanol extracts the future for the prevention of medical and biomedical implant contaminations? Phytomedicine. Jul 1, 2017, Vol. 30, pp. 50-58.

42. Diamond, G., et al. The Roles of Antimicrobial Peptides in Innate Host Defense. Curr Pharm Des. 2009; 15(21):. 2009, Vol. 15, 21, pp. 2377-2392.

43. Matsuzaki, K. Control of cell selectivity of antimicrobial peptides.Biochimica et Biophysica Acta (BBA) - Biomembranes. Aug, 2009, Vol. 1788, 8.

44. Osés, S.M., et al. Bioactive properties of honey with propolis.Food Chem. 2016.

45. Renneberg, R.; Berkling, V.; Loroc, V. Biotechnology for Beginners, ISBN:9780128012734. 2nd Edition. 2016. pp. 197-198.

46. Zaobidna, E.A.; Żółtowska, K.; Łopieńska-Biernat, E. Varroa destructor induces changes in the expression of immunity-related genes during the development of Apis mellifera worker and drone broods.Acta Parasitologica | Volume 62: Issue 4.DOI: https://doi.org/10.1515/ap-2017-0094 | Published online: Oct 15, 2017.

47. BATISTA, E.K.F.; et al. Influence of Propolis on leukocyte and protein profiles of mice and closing time of excisional wounds clean and infected by Staphylococcus aureus. Rev. bras. plantas med. vol.17 no.3 Botucatu jul./set. 2015. Vol. 17. 
48. Touzani, S.; et al. In Vitro Evaluation of the Potential Use of Propolis as a Multitarget Therapeutic Product: Physicochemical Properties, Chemical Composition, and Immunomodulatory, Antibacterial, and Anticancer Properties. Biomed Res Int. 2019, Vol. 4836378.

49. Bayrami, M.; et al. Medicinal effects of Propolis diet on biochemicals and histological factors in rats. Trends Med. 2019, Vol. 19, DOI: 10.15761/TiM.1000213.

50. Wong, K. TakePart. TakePart. [Online] Participant Media, Oct 29, 2014. [Cited on: Apr 08, 2020.] http://www.takepart.com/article/2014/10/29/bugs-could-save-bees.

51. Drescher, N.; et al. Inside Honeybee Hives: Impact of Natural Propolis on the Ectoparasitic Mite Varroa destructor and Viruses.

Insects. 2017 Mar; 8(1): 15.

52. Pusceddu, M.; et al. The effects of raw propolis on Varroa-infested honey bee (Apis mellifera) workers. Parasitol Res. 2018 Nov;117(11):3527-3535.

53. Kumar, P.; Kizhakkedathu, J.N.; Straus, SK. Antimicrobial Peptides: Diversity, Mechanism of Action and Strategies to Improve the Activity and Biocompatibility In Vivo. Biomolecules. 8(1), 2018, Vol. 4.

54. Mahlapuu, M.; Håkansson, J; et al. Antimicrobial Peptides: An Emerging Category of Therapeutic Agents. Front Cell Infect Microbiol. 2016, Vol. 6, 194.

55. Reddy, K.V.; Yedery, R.D.; Aranha, C. Antimicrobial peptides: premises and promises. Int J Antimicrob Agents. Dec, 2004, Vol. 24, 6, pp. 536-47.

56. Wang, G. Human antimicrobial peptides and proteins.

Pharmaceuticals (Basel). 2014, May 13;7(5):545-94.

57. Oyinloye, B.E.; Adenowo, A.F.; Kappo, A.P. Reactive Oxygen Species, Apoptosis, Antimicrobial Peptides and Human Inflammatory Diseases.

Pharmaceuticals (Basel). 2015 Jun; 8(2): 151-175.

58. Jun, L.; et al. The antimicrobial peptides and their potential clinical applications. Am J Transl Res. 7 , 2019, Vol. 11, pp. 3919-3931.

59. Hancock, R.E.; Haney, E.F.; Gill, E.E. The immunology of host defence peptides: beyond antimicrobial activity. Nat Rev Immunol.May 16, 2016, Vol. 5, pp. 321-34.

60. Elbourne, A.; Cheeseman, S.; Atkin, P. Antibacterial Liquid Metals: Biofilm Treatment via Magnetic Activation. ACS Nano. 2020.

61. Nguyen, L.T.; Haney, E.F.; Vogel, H.J. The expanding scope of antimicrobial peptide structures and their modes of action. Trends Biotechnol. Sep 29, 2011, Vol. 9, pp. 464-72.

62. Bechinger, B.; Lohner, K. Detergent-like actions of linear amphipathic cationic antimicrobial peptides. Biochimica et Biophysica Acta (BBA) - Biomembranes. Sept, 2006, Pages, Vol. 1758, 9, pp. 1529-1539.

63. Scott, M.G.; Hancock, R.E. Cationic antimicrobial peptides and their multifunctional role in the immune system. Crit Rev Immunol.2000, Vol. 20, 5, pp. 407-31.

64. Mogensen, T.H.; Paludan, S.R. Molecular Pathways in Virus-Induced Cytokine Production. Microbiol Mol Biol Rev. 65, Mar, 2001, Vol. 1, pp. 131-150.

65. Bahar, A.A.; Ren, D. Antimicrobial Peptides. Pharmaceuticals (Basel). 2013 Dec; 6(12): 1543-1575.

66. Sulaiman, C.T.; Balachandran, I. Total Phenolics and Total Flavonoids in Selected Indian Medicinal Plants.Indian J Pharm Sci. 2012 May-Jun; 74(3): 258-260. 
67. Galeotti, F., et al. Chemical Composition and Antioxidant Activity of Propolis Prepared in Different Forms and in Different Solvents Useful for Finished Products. Foods. 2018 Mar; 7(3): 41.

68. Bull, H.B.; Breese, K. Interaction of alcohols with proteins.

Biopolymers. Volume17, Issue 9. Sep 1978, Pages 2121-2131. https://doi.org/10.1002/bip.1978.360170907.

69. Szliszka, E.; et al. Inhibition of Inflammatory Response by Artepillin C in Activated RAW264.7 Macrophages. Research Article. Open Access. 2013. Article ID 735176. https://doi.org/10.1155/2013/735176.

70. Shimizu, K.; et al. Antioxidative bioavailability of artepillin C in Brazilian propolis.Arch Biochem Biophys. 2004, Apr 15;424(2):181-8.

71. Messerli, S.M.; Ahn, M.R.; Kunimasa, K.; et al. (2009), Artepillin C (ARC) in Brazilian green propolis selectively blocks oncogenic PAK1 signaling and suppresses the growth of NF tumors in mice. Phytother. Res., 23: 423-427. doi:10.1002/ptr.2658.

72. Endo, S.; et al. Autophagy inhibition enhances anticancer efficacy of artepillin C, a cinnamic acid derivative in Brazilian green propolis. Biochemical and Biophysical Research Communications.Volume 497, Issue 1, February 26, 2018, Pages 437-443.

73. Yoshimasu, Y., et al. Rapid Bactericidal Action of Propolis against Porphyromonas gingivalis.J Dent Res. 2018 Jul;97(8):928-936. doi: 10.1177/0022034518758034. Epub 2018, Mar 1.

74. Figueiredo-Rinhel, A.S.G.; de Melo, L.L.; Bortot, L.O.; Santos, E.O.L.; Andrade, M.F.; Azzolini, A.E.C.S.; Kabeya, L.M.; Caliri, A.; Bastos, J.K.; Lucisano-Valim, Y.M. (2017), Baccharis dracunculifolia DC (Asteraceae) selectively modulates the effector functions of human neutrophils. J Pharm Pharmacol, 69: 1829-1845. doi:10.1111/jphp.12822.

75. Kumazak, I. M.; Shinohara, H.; Taniguchi, K.; et al. Propolis Cinnamic Acid Derivatives Induce Apoptosis Through Both Extrinsic and Intrinsic Apoptosis Signaling Pathways and Modulate of miRNA Expression.

Phytomedicine. Jul-Aug 2014;21(8-9):1070-7. doi: 10.1016/j.phymed.2014.04.006. Epub 2014 May 20.

76. Sova, M. Antioxidant and Antimicrobial Activities of Cinnamic Acid Derivatives. Mini Rev Med Chem. 2012 Jul;12(8):749-67. doi: 10.2174/138955712801264792.

77. Laverty, G., et al. Anti-biofilm activity of ultrashort cinnamic acid peptide derivatives against medical device-related pathogens.J Pept Sci. 2015 Oct;21(10):770-8. doi: 10.1002/psc.2805. Epub 2015 Aug 27.

78. Ghalehshahi, H.G.; Balalaie, S.; Aliahmadi, A. Peptides N-connected to hydroxycoumarin and cinnamic acid derivatives: synthesis and fluorescence spectroscopic, antioxidant and antimicrobial properties.

New J. Chem., 2018,42, 8831-8842. https://doi.org/10.1039/C8NJ00383A.

79. Ming, N.; Shuangmu Z. Applications of self-assembling ultrashort peptides in bionanotechnology. RSC Adv., 2019,9, 844-852. https://doi.org/10.1039/C8RA07533F.

80. Adisakwattana, S. Cinnamic Acid and Its Derivatives: Mechanisms for Prevention and Management of Diabetes and Its Complications.

Nutrients. 2017 Feb; 9(2): 163. doi: 10.3390/nu9020163.

81. Wiese, N.; Fischer, J.; Heidler, J.; et al. The terpenes of leaves, pollen, and nectar of thyme (Thymus vulgaris) inhibit growth of bee disease-associated microbes. Sci Rep 8, 14634 (2018). https://doi.org/10.1038/s41598-018-32849-6.

82. Aminimoghadamfarouj, N.; Nematollahi, A. Propolis Diterpenes as a Remarkable Bio-Source for Drug Discovery Development: A Review. 
Int J Mol Sci. 2017 Jun; 18(6): 1290. doi: 10.3390/ijms18061290.

83. Mahizan, N.A.; Yang, S.K.; Moo, C.L., et al. Terpene Derivatives as a Potential Agent against Antimicrobial Resistance (AMR) Pathogens.

Molecules. 2019 Jul; 24(14): 2631. doi: 10.3390/molecules24142631.

84. Urgert, R.; Weusten-van der Wouw, M.P.; Hovenier, R., et al. Diterpenes From Coffee Beans Decrease Serum Levels of Lipoprotein(a) in Humans: Results From Four Randomised Controlled Trials. Eur J Clin Nutr. 1997 Jul;51(7):431-6. doi: 10.1038/sj.ejcn.1600414.

85. Booth, J.K.; Bohlmann, J. Terpenes in Cannabis sativa - From plant genome to humans. Plant Science. Volume 284, July 2019, Pages 67-72. https://doi.org/10.1016/j.plantsci.2019.03.022.

86. Menon S.; Chunxia L.; Menon R.; et al. Effects of Antioxidants in Human Cancers: Differential Effects on Non-Coding Intronic RNA Expression. Antioxidants (Basel). 2016 Mar; 5(1): 1. doi: 10.3390/antiox 5010001.

87. How antioxidants can accelerate cancers, and why they don't protect against them. Science Daily.Science News. Source: Cold Spring Harbor Laboratory. https://www.sciencedaily.com/releases/2014/07/140710094434.htm. Jul 10, 2014.

88. Kardar, M.N.; Zhang, T.; Coxon, G.D.; Watson, D.G.; Fearnley, J.; Seidel, V. Characterisation of triterpenes and new phenolic lipids in Cameroonian propolis. Phytochemistry. Volume 106, October 2014, Pages 156-163. https://doi.org/10.1016/j.phytochem.2014.07.016.

89. Stasiuk, M.; Kozubek, A. Membrane perturbing properties of natural phenolic and resorcinolic lipids. FEBS Letters. Volume 582, Issues 25-26, October 29, 2008, Pages 3607-3613. https://doi.org/10.1016/j.febslet.2008.09.039.

90. Stasiuk, M.; Kozubek, A. Biological activity of phenolic lipids.

Cell. Mol. Life Sci. 67, 841-860 (2010). https://doi.org/10.1007/s00018-009-0193-1.

91. Chibane, L.B.; et al. Antibacterial Properties of Polyphenols: Characterization and QSAR (Quantitative Structure-Activity Relationship) Models. Front. Microbiol., April, 18 2019| https://doi.org/10.3389/fmicb.2019.00829.

92. Mauricio, A.; de Paula, B.; et al. Susceptibility of Oral Pathogenic Bacteria and Fungi to Brazilian Green Propolis Extract. .

Pharmacologyonline 3: 467-473 (2006).

93. Kampf, G. Efficacy of ethanol against viruses in hand disinfectionEfficacy of ethanol against viruses in hand disinfectionEfficacy of ethanol against viruses in hand disinfection.

Journal of Hospital Infection. Vol. 98, issue 4, Pgs. 331-338, April 1, 2018.

94. Fraise, AP; et al. The antibacterial activity and stability of acetic acid. Journal of Hospital Infection. Volume 84, Issue 4, August 2013, Pages 329-331.

95. Terasaki, M.; Ito, H.; Kurokawa, H; etal. Acetic acid is an oxidative stressor in gastric cancer cells. $J$ Clin Biochem Nutr. 2018 Jul; 63(1): 36-41.

96. Nurmi, A.; Nurmi, T; Mursu, J.; et al. Ingestion of Oregano Extract Increases Excretion of Urinary Phenolic Metabolites in Humans.

Journal of Agricultural and Food Chemistry 200654 (18), 6916-6923. 
97. Escalante-Perez M.; Jaborsky M.; Reindersb J.; Kurzaic O.; Ache P. Poplar Extrafloral Nectar Is Protected against Plant and Human Pathogenic Fungus. Molecular Plant. Volume 5, Number 5, Pages 1157-1159. Sep, 2012.

98. Ramada, M.H.S.; Brand, G.D.; Abrao, F.Y.; et al. Encrypted Antimicrobial Peptides from Plant Proteins. Sci Rep. 2017; 7: 13263.doi: 10.1038/s41598-017-13685-6.

99. Nawrot, R.; Barylski, J.; Nowicki, G.; et al. Plant antimicrobial peptides. Folia Microbiol (2014) 59:181-196. DOI 10.1007/s12223-013-0280-4.

100. Chen, C.H.; Lu, T.K. Development and Challenges of Antimicrobial Peptides for Therapeutic Applications. Antibiotics 2020, 9, 24.

101. Ross, C.F.; Hoye, C. Jr.; Fernandez-Plotka, V.C. Influence of heating on the polyphenolic content and antioxidant activity of grape seed flour. J Food Sci. 2011 Aug;76(6):C884-90. doi: 10.1111/j.17503841.2011.02280.x.

102. Ebbensgaard, A.; Mordhorst, H.; Overgaard, M.T.; et al. Comparative Evaluation of the Antimicrobial Activity of Different Antimicrobial Peptides against a Range of Pathogenic Bacteria. PLoS One. 2015; 10(12): e0144611.

103. Ararso, Z.; Legesse, G. Insecticidal action of honeybees propolis extract against larvae of lesser. AGRICULTURE AND BIOLOGY JOURNAL OF NORTH AMERICA, doi:10.5251/abjna.2016.7.6.302.306. 2016.

104. Haynie, S.L.; Crum, G.A.; Doele, B.A. Antimicrobial activities of amphiphilic peptides covalently bonded to a water-insoluble resin.

Antimicrob Agents Chemother. 1995 Feb; 39(2): 301-30\%.doi: 10.1128/aac.39.2.301.

105. Cornell University. "Two Studies On Bee Evolution Reveal Surprises." ScienceDaily. ScienceDaily, 14 December 2006.<www.sciencedaily.com/releases/2006/12/061209083342.htm>.

106. Danihlik, J.; Aronstein, K; Petřivalský, M. Antimicrobial peptides: a key component of honey bee innate immunity, Journal of Apicultural Research, 54:2, 123-136, DOI: 10.1080/00218839.2015.1109919. 2015.

107. Peng, X.; Min, S.; Xue-xin, C. Antimicrobial Peptide Evolution in the Asiatic Honey Bee Apis cerana. PLoS One. 2009; 4(1): e4239.

108. Kim, D.S.; et al. A new prokaryotic expression vector for the expression of antimicrobial peptide abaecin using SUMO fusion tag.BMC Biotechnology volume 19, Article number: 13 (2019).https://doi.org/10.1186/s12896-019-0506-x.

109. Knappe, D.; et al. Continuous Subcutaneous Delivery of Proline-Rich Antimicrobial Peptide Api137 Provides Superior Efficacy to Intravenous Administration in a Mouse Infection Model. Front. Microbiol., October 02, 2019 | https://doi.org/10.3389/fmicb.2019.02283.

110. Florin, T; et al. An antimicrobial peptide that inhibits translation by trapping release factors on the ribosome.Nat Struct Mol Biol. 2017 Sep;24(9):752-757. doi: 10.1038/nsmb.3439. Epub 2017 Jul 24.

111. Luiz, D.P.; Almeida, J.F.; Goulart, L.R.; et al. Heterologous expression of abaecin peptide from Apis mellifera in Pichia pastoris. Heterologous expression of abaecin peptide from Apis mellifera in Pichia pastoris . Microb Cell Fact 16, 76 (2017). https://doi.org/10.1186/s12934-017-0689-6.

112. Holfeld, L.; Knappe, D.; Hoffmann, R. Proline-rich antimicrobial peptides show a long-lasting postantibiotic effect on Enterobacteriaceae and Pseudomonas aeruginosa. Journal of Antimicrobial Chemotherapy, Volume 73, Issue 4, April 2018, Pages 933-941, https://doi.org/10.1093/jac/dkx482.

113. Graf M.; Mardirossian M.; Nguyen F.; et al. Proline-rich Antimicrobial Peptides Targeting Protein Synthesis. Nat Prod Rep. 2017;34(7):702-711. doi:10.1039/c7np00020k. 
114. Florin, T.; Maracci, C.; Graf, M.; et al. An antimicrobial peptide that inhibits translation by trapping release factors on the ribosome.

Nat Struct Mol Biol 24, 752-757 (2017). https://doi.org/10.1038/nsmb.3439.

115. Ruppenthal, A. WTTW NEWS. WTTW News. [Online] WTTW , Sep, 2017. [Cited on: Apr 26, 2020.] https://news.wttw.com/2017/09/12/honeybees-could-produce-next-new-antibiotics-study-says.

116. Rahnamaeian, M.; et al. Insect antimicrobial peptides show potentiating functional interactions against Gram-negative bacteria. Proc Biol Sci. 2015 May 7; 282(1806): 20150293.

117. Yu, G.; Baeder, D.Y.; Regoes R.R.; Rolff J. 2016. Combination effects of antimicrobial peptides. Antimicrob Agents Chemother 60:1717-1724. doi:10.1128/AAC.02434-15. [Online]

118. Rustem, A.I.; Louisa, R.G. Review of the Expression of Antimicrobial Peptide. Journal of Apicultural Science. 2012, Vol. Vol. 56 No. 1.

119. Zhao, B.C.; Lin, H.C., Yang, D.; Ye, X.; Li, Z.G. Disulfide Bridges in Defensins. Curr Top Med Chem. 2015;16(2):206-219. doi:10.2174/1568026615666150701115911.

120. Bucekova, M.; Sojka, M.; Valachova, I.; et al. Bee-derived antibacterial peptide, defensin-1, promotes wound re-epithelialisation in vitro and in vivo . Sci Rep 7, 7340 (2017). https://doi.org/10.1038/s41598-01707494-0.

121. Nolan, V.C.; Harrison, J.; Jonathan A. G. Cox. Dissecting the Antimicrobial Composition of Honey. Antibiotics 2019, 8(4), 251; https://doi.org/10.3390/antibiotics8040251. Crossref.

122. Foster, K. There Are Shocking Differences Between Raw Honey and the Processed Golden Honey Found in Grocery Retailers. Permaculture news. [Online] Permaculture Research Institute, Feb 8, 2014. [Cited in: April 18, 2020.] https://www.permaculturenews.org/2014/02/08/shocking-differences-raw-honey-processedgolden-honey-found-grocery-retailers/.

123. Brudzynski, K.; Sjaarda, C.; Lannigan, R. MRJP1-containing glycoproteins isolated from honey, a novel antibacterial drug candidate with broad spectrum activity against multi-drug resistant clinical isolates. Front Microbiol. 2015; 6: 711. Published online 2015, Jul 13. doi: 10.3389/fmicb.2015.00711.

124. Brudzynsk K.; Sjaarda C. Honey Glycoproteins Containing Antimicrobial Peptides, Jelleins of the Major Royal Jelly Protein 1, Are Responsible for the Cell Wall Lytic and Bactericidal Activities of Honey. Plos One. https://doi.org/10.1371/journal.pone.0120238.April 1, 2015.

125. Gannabathula S.; Krissansen G.W.; Skinner M.; Steinhorn G.; Schlothauer R. Honeybee apisimin and plant arabinogalactans in honey costimulate monocytes. Food Chem. 2015 Feb 1;168:34-40. doi: 10.1016/j.foodchem.2014.07.007. Epub 2014 Jul 9.

126. Furusawa, T.; Arai, Y.; Kato, K.; Ichihara, K. Quantitative Analysis of Apisin, a Major Protein Unique to Royal Jelly.

Hindawi. Research Article. Open Access.Volume 2016 |Article ID 5040528 | 9 pages | https://doi.org/10.1155/2016/5040528.

127. Buttstedt, A.; Moritz R.F.; Erler S. Origin and function of the major royal jelly proteins of the honeybee (Apis mellifera) as members of the yellow gene family. Biological Reviews of the Cambridge Philosophical Society. 89 (2): 255-69. doi:10.1111/brv.12052. May, 2014.

128. Albert S.; Bhattacharya D.; Klaudiny J.; Schmitzová J.; Simúth J. The family of major royal jelly proteins and its evolution.Journal of Molecular Evolution. 49 (2): 290-7. doi:10.1007/PL00006551. Aug, 1999.

129. Park, M.J.; Kim, B.Y.; et al. Major royal jelly protein 2 acts as an antimicrobial agent and antioxidant in royal jelly. Journal of Asia-Pacific Entomology. Volume 22, Issue 3, September 2019, Pages 684-689. 
130. Gannabathula S.; Skinner M.A.; Rosendale D.; et al. Arabinogalactan proteins contribute to the immunostimulatory properties of New Zealand honeys, Immunopharmacology and Immunotoxicology, 34:4, 598-607, DOI: 10.3109/08923973.2011.641974. 2012.

131. Singha P.K.; Roy S; Dey S. Antimicrobial activity of Andrographis paniculata. Fitoterapia. 2003 Dec;74(7-8):692-4.

132. Baien S.H.; Seele J.; Henneck T.; et al. Antimicrobial and Immunomodulatory Effect of Gum Arabic on Human and Bovine Granulocytes Against Staphylococcus aureus and Escherichia coli. Front. Immunol., 31 January 2020 | https://doi.org/10.3389/fimmu.2019.03119.

133. Krempel M.; Griffin K.; Khouryieh H. Preservatives and Preservation Approaches in Beverages. https://doi.org/10.1016/B978-0-12-816685-7.00013-6. 2019. pp. 427-465. Vol. 5: the Science of Beverages.

134. Sasu M.A.; Wall K.L.; Stephenson A.G. Antimicrobial nectar inhibits a florally transmitted pathogen of a wild Cucurbita pepo (Cucurbitaceae). Am J Bot. 2010 Jun;97(6):1025-30. doi: 10.3732/ajb.0900381. Epub 2010 May 21.

135. Hillwig M.S.; Xiaoteng L.; Guangyu L.; Thornburg R.W.; MacIntosh G.C. Petunia nectar proteins have ribonuclease activity. Journal of Experimental Botany, Volume 61, Issue 11, June 2010, Pages 2951-2965, https://doi.org/10.1093/jxb/erq119.

136. Escalante-Pérez M.; Jaborsky M.; Lautner S.; Fromm J.; et al. Poplar Extrafloral Nectaries: Two Types, Two Strategies of Indirect Defenses against Herbivores. Plant Physiol. 2012 Jul; 159(3): 1176-1191.Published online 2012 May 9.doi: 10.1104/pp.112.196014.

137. Carter, C.; Shafir, S.; Yehonatan, L.; Palmer, R. G.; Thornburg, R. A novel role for proline in plant floral nectars.Naturwissenschaften, 93(2), 72-79. https://doi.org/10.1007/s00114-005-0062-1. 2006.

138. Nepi M.; Grasso D.A.; Mancuso, S. Nectar in Plant-Insect Mutualistic Relationships: From Food Reward to Partner Manipulation.

Front. Plant Sci., 19 July 2018 | https://doi.org/10.3389/fpls.2018.01063. Cross-ref.

139. Cornara L.; Biagi M.; Xiao J.; Burlando B. Therapeutic Properties of Bioactive Compounds from Different Honeybee Products. Front. Pharmacol., 28 June 2017| https://doi.org/10.3389/fphar.2017.00412.

140. Hu H.; Bezabih G.; Feng M.; et al. In-depth Proteome of the Hypopharyngeal Glands of Honeybee Workers Reveals Highly Activated Protein and Energy Metabolism in Priming the Secretion of Royal Jelly.

Molecular 83 Cellular Proteomics April 1, 2019, First published on January 7, 2019, 18 (4) 606-621; https://doi.org/10.1074/mcp.

141. Vagish, S.; Kumar, L. Propolis in Dentistry and Oral Cancer Management. N Am J Med Sci. 2014 Jun; 6(6): 250-259. Cross ref.

142. Zhang C.P.; Zheng H.Q; Hu F.L. Extraction, partial characterization, and storage stability of $\beta$ glucosidase from propolis.

J Food Sci. 2011 Jan-Feb;76(1):C75-9. doi: 10.1111/j.1750-3841.2010.01941.x.

143. Lopez-Uribe, M.M.; Simone-Finstrom, M. Glucose oxidase production does not increase after colony infection: Testing its role in honey bee social immunity. Bee World. 93(4):104-127. 2017.

144. Vannette, R.; Mohamed, A.; Johnson, B. Forager bees (Apis mellifera) highly express immune and detoxification genes in tissues associated with nectar processing. Sci Rep 5, 16224 (2015). https://doi.org/10.1038/srep16224.

145. Jasper, W.C.; Linksvayer, T.A.; Atallah, J.; Friedman, D.; Chiu, J.C.; Johnson, B.R. Large-Scale Coding Sequence Change Underlies the Evolution of Postdevelopmental Novelty in 
Honey Bees. Molecular Biology and Evolution, Volume 32, Issue 2, February 2015, Pages 334346,https://doi.org/10.1093/molbev/msu292.

146. Ohashi, K.; Natori, S.; Kubo, T. Expression of amylase and glucose oxidase in the hypopharyngeal gland with an age-dependent role change of the worker honeybee. Eur. J. Biochem. 265, 127-133 (1999).

147. Danihlík, J.; Škrabišová, M.; Lenobel, R.; et al. Does the Pollen Diet Influence the Production and Expression of Antimicrobial Peptides in Individual Honey Bees? Insects. 2018 Sep; 9(3): 79. doi: 10.3390/insects9030079.

148. Marcucci, M.C.; de Camargo, F.A.; Lopes, C.M.A. Identification of Amino Acids in Brazilian Propolis. Z. Naturforsch. 51c, 11 -1 4 (1996); received March 27/October 12, 1995.

149. Eroglu, N.; Akkus, S.; Yaman, M.; Asci, B.; Silici, S. Amino Acid and Vitamin Content of Propolis Collected by Native Caucasican Honeybees. Journal of Apicultural Science. (2016) 60. 10.1515/JAS-20160021. .

150. Gabrys J.; Konecki J.; Krol W.; Scheller S.; Shani J. Free amino acids in bee hive product (propolis) as identified and quantified by gas-liquid chromatography. Pharmacol Res Commun. 1986 Jun;18(6):513-8.

151. Cutrona, K.J.; Kaufman, B.A.; Figueroa, D.M.; Elmore, D.E. Role of Arginine and Lysine in the Antimicrobial Mechanism of Histone-derived Antimicrobial Peptides. FEBS Lett. 2015 Dec 21; 589 (24 0 0): 3915-3920.doi: 10.1016/j.febslet.2015.11.002.

152. Anbanandam, A.; Albarado, D.C.; Tirziu, D.C.; Simons, M.; Veeraraghavan, S. Molecular Basis for Proline- And Arginine-Rich Peptide Inhibition of Proteasome. J Mol Biol.2008 Dec 5;384(1):219-27. doi: 10.1016/j.jmb.2008.09.021. Epub 2008 Sep 16.

153. Ntwasa, M. Cationic Peptide Interactions with Biological Macromolecules. Intechopen open access peerreviewed chapter.Published: September 19th 2012. DOI: 10.5772/48492.

154. Hindi, N.; Al-Charrakh, A.; Saheb Naer, H.; Abbas, A. (2015). STUDY OF CHEMICAL ANALYSIS OF IRAQI PROPOLIS AND ACTIVE COMPONENT OF PROPLIS, IRAQ. Journal of Science Microbiology 2277 - 3290. 5. 1095-1103. .

155. Yu-Fen, C.; Chao-Hsun, Y.; Ming-Shiang, C.; et al. Foam Properties and Detergent Abilities of the Saponins from Camellia oleifera.

Int J Mol Sci. 2010; 11(11): 4417-4425.doi: 10.3390/ijms11114417.

156. Kuropatnicki, A.K; Szliszka, E.; Krol, W. Historical Aspects of Propolis Research in Modern Times. Hindawi. https://doi.org/10.1155/2013/964149. April 28, 2013.

157. Aufderheide, A. C. The Scientific Study of Mummies. s.l. : Cambridge University Press, 2003. p. 46.

158. Geiling, N. The Science Behind Honey's Eternal Shelf Life. Smithsonianmag.com. Aug. 22, 2013. https://www.smithsonianmag.com/science-nature/the-science-behind-honeys-eternal-shelf-life-1218690/.

159. Berg, J.M.; Tymoczko, J.L.; Stryer, L. Biochemistry, 5th edition. https://www.ncbi.nlm.nih.gov/books/NBK22526/. New York : WH Freeman, 2002.

160. Yeagle, P.L. The Membranes of Cells (Third Edition).2016, Pages 73-84. https://doi.org/10.1016/B9780-12-800047-2.00004-8.

161. Roth, M. Pittsburgh Post-Gazette. Pittsburgh Post-Gazette website. [Online] Feb 17, 2010. [Cited on: Apr 29, 2020.] https://www.post-gazette.com/news/2010/02/17/Researcher-says-physics-may-outsmartviruses/stories/201002170233.

162. Wang, G. Natural antimicrobial peptides as promising anti-HIV candidates. Curr Top Pept Protein Res. 2012; 13: 93-110. 
163. Le, W.; Chen, B.; Cui, Z.; et al. Detection of cancer cells based on glycolytic-regulated surface electrical charges. Biophys Rep 5, 10-18 (2019). https://doi.org/10.1007/s41048-018-0080-0.

164. Premratanachai, P.; Chanchao, C. Review of the anticancer activities of bee products. Asian Pac $J$ Trop Biomed. 2014 May; 4(5): 337-344. doi: 10.12980/APJTB.4.2014C1262.

165. Cauvi D.M.; Hawisher D.; Dores-Silva P.R.; Lizardo R.E.; De Maio, A. Macrophage reprogramming by negatively charged membrane phospholipids controls infection. FASEB J. 2019;33(2):2995-3009. doi:10.1096/fj.201801579R.

166. Diaz, F.R.; Dantas, E.; Geffner, J. Unravelling the Interplay between Extracellular Acidosis and Immune Cells. Hindawi. Volume 2018.Article ID 1218297. 11 pages. https://doi.org/10.1155/2018/1218297.

167. Malik, E.; Dennison, S.R.; Harris, F.; Phoenix, D.A. pH Dependent Antimicrobial Peptides and Proteins, Their Mechanisms of Action and Potential as Therapeutic Agents. Pharmaceuticals (Basel). 2016 Nov 1;9(4):67. doi: 10.3390/ph9040067.

168. Tagliacollo, V.A.; Orsi, R.O. Quality of propolis commercialized in the informal market. Food Science and Technology. vol.31 no.3 Campinas July/Sept. 2011. https://doi.org/10.1590/S010120612011000300031.

169. Sinha, R.; Shukla, P. Antimicrobial Peptides: Recent Insights on Biotechnological Interventions and Future Perspectives. Protein Pept Lett. 2019 Feb; 26(2): 79-87.doi: 10.2174/0929866525666181026160852.

170. World Health Organization. 29 April 2019 Joint News Release New York. [Online] Joint News Release New York, April 29, 2019. [Cited on: April 9, 2020.] https://www.who.int/news-room/detail/29-04-2019new-report-calls-for-urgent-action-to-avert-antimicrobial-resistance-crisis.

171. The Global Impact of Respiratory Disease. Second Edition. Sheffi eld, European Respiratory Society, $201 \%$.

172. ScienceDaily . ScienceDaily Science news website.[Online] RMIT University, Jan 13, 2020. [Cited on: April 30, 2020.] https://www.sciencedaily.com/releases/2020/01/200113111056.htm.

173. Ivanovska N.D.; Dimov V.B.; Bankova V.S.; Popov S.S. Immunomodulatory action of propolis. VI. Influence of a water soluble derivative on complement activity in vivo. J Ethnopharmacol. 1995;47(3):145147. doi:10.1016/0378-8741(95)01272-f.

174. Farooqui, T.; Farooqui, A.A. Beneficial effects of propolis on human health and neurological diseases. Front.Biosci. 4, 2012, pp. 779-793.

175. Jartti, T.; et al. Serial viral infections in infants with recurrent respiratory illnesses. Eur Respir $J$ 32(2): Aug 2008, pp. 314-320.

176. Guney, A.; et al. Effects of Propolis on Fracture Healing: An Experimental Study. Phitoterapy Research. 11, Nov 2011, Vol. 25.

177. Burdock, G.A. Review of the biological properties and toxicity of bee propolis (propolis). Food Chem Toxicol. Apr, 1998, Vol. 36, 4, pp. 347-63.

178. Coelho, L.G.; et al. Brazilian green propolis on Helicobacter pylori infection. A pilot clinical study. Helicobacter. 5. Oct 12, 2007, Vol. 12, pp. 572-4.

179. Arvouet-Grand, A.; et al. Propolis extract. I. Acute toxicity and determination of acute primary cutaneous irritation index. J Pharm Belg. 3, May-Jun, 1993, Vol. 48, pp. 165-70.

180. Miner-Williams, W.M.; Stevens, B.R.; Moughan, P.J. Are Intact Peptides Absorbed From the Healthy Gut in the Adult Human? Nutr Res Rev.2014 Dec;27(2):308-29. doi: 10.1017/S0954422414000225. 
181. Nunes, D.D.; et al. Chemical characterization and biological activity of six different extracts of propolis through conventional methods and supercritical extraction. Plos one. Dec 4, 2018.

182. Kubiliene, L.; et al. Comparison of aqueous, polyethylene glycol-aqueous and ethanolic propolis extracts: antioxidant and mitochondria modulating properties. BMC Complementary and Alternative Medicine. 2018, Vol. 18, p. 165.

183. Kubiliene, L..; et al. Alternative preparation of propolis extracts: comparison of their composition and biological activities.BMC Complement Altern Med. May 27, 2015, Vol. 15, p. 156.

184. Apis Flora. cienciadapropolis.com.br. [Online] Apis Flora, 2019. [Cited on: Apr 3, 2020.] https://cienciadapropolis.com.br/en/what-is-epp-af/epp-af/.

185. Google Patents. Google. [Online] Google. [Cited on: Apr 3, 2020.] https://patents.google.com/patent/US5922324A/en.

186. Chang, S.R.; et al. Microsomes, Inhibition of Cytochrome P450 by Propolis in Human Liver. Toxicol Res. 3, Jul 30, 2016, Vol. 32, pp. 207-213.

187. Cusinato, D.A.C.; et al. Evaluation of potential herbal-drug interactions of a standardized propolis extract (EPP-AF(r)) using an in vivo cocktail approach. Journal of Ethnopharmacology. aug 19, 2019, Vol. 245, p. 112174.

188. dos Santos, D.A.; Munaria, F.M.; Frozza, C.O.S.; et al. Brazilian red propolis extracts: study of chemical composition by ESI-MS/MS (ESI+) and cytotoxic profiles against colon cancer cell lines.

Biotechnology Research and Innovation. Volume 3, Issue 1, January-June 2019, Pages 120-130.

189. Calhelha, R.C.; Falcao,S.; Queiroz, M.J.R.P.; et al. Cytotoxicity of Portuguese Propolis: The Proximity of the In Vitro Doses for Tumor and Normal Cell Lines. Hindawi. Open access article. Volume 2014. Article ID 897361. 7 pages. https://doi.org/10.1155/2014/897361.

190. Martinotti, S.; Ranzato, E. Propolis: a new frontier for wound healing? Burns Trauma. 2015; 3: 9. Published online 2015 Jul 22. doi: 10.1186/s41038-015-0010-z.

191. Hirota, R.; Ngatu, N.R.; Nakamura, H.; Suganuma, N. Propolis Inhalation Reduces Allergic Airway Inflammation in Dermatophagoides Farinae -Treated Mice. . 2012 International Conference on Nutrition and Food Sciences. IPCBEE vol. 39 (2012) IACSIT Press, Singapore.

192. Bogdanov, S. Propolis: biological properties and medical applications. Bee Product Science. Cross ref. https://www.researchgate.net/publication/304012147_Propolis_biological_properties_and_medical_applications.2016.

193. Parashar, P.; Agrawal, O.P. Ethnomedicinal properties of propolis and its usages in historic zootherapeutic practices. International Journal of Zoology Studies. Volume 3; Issue 2; March 2018; Page No. 235-238. ISSN: 2455-7269. .

194. Patton, J.; Byron, P. Inhaling medicines: delivering drugs to the body through the lungs. Nat Rev Drug Discov 6, 67-74 (2007). https://doi.org/10.1038/nrd2153.

195. Patton, J.S.; Fishburn, C.S.; Weers, J.G. The Lungs as a Portal of Entry for Systemic Drug Delivery. AtsJournal, Volume 1, Issue 4. https://www.atsjournals.org/doi/full/10.1513/pats.200409-049TA. 2004.

196. Beisswenger, C.; Bals, R. Antimicrobial Peptides in Lung Inflammation. Chem Immunol Allergy.2005;86:55-71. doi: 10.1159/000086651.

197. Sethi, S.; Bryan, J. Antibiotic resistance: challenges and successes in respiratory infection. Future Microbioloogy. Vol. 11, n. 4. Conference report.15 Apr 2016. https://doi.org/10.2217/fmb-2016-0006. 
198. Ya-Min, Y.; Yi-Dong L.; Xing-Lei, S.; et al. Therapeutic Effects of Inhaling Aerosolized Surfactant Alone or With Dexamethasone Generated by a Novel Noninvasive Apparatus on Acute Lung Injury in Rats. J Trauma Acute Care Surg. 2012 Nov;73(5):1114-20. doi: 10.1097/TA.0b013e318265cbe9.

199. What Other Coronaviruses Tell Us About SARS-CoV-2.Quanta Magazine. April 29, 2020. https://www.quantamagazine.org/what-can-other-coronaviruses-tell-us-about-sars-cov-2-20200429/\#.Smith, T.C.

200. GeneTex. GeneTex website. [Online] GeneTex, Inc. [Cited on: May 3, 2020.] https://www.genetex.com/Research/Overview/infectious_diseases/SARS-CoV-2.

201. Shang, W.; Yang, Y.; Rao, Y.; et al. The outbreak of SARS-CoV-2 pneumonia calls for viral vaccines. npj Vaccines 5, 18 (2020). https://doi.org/10.1038/s41541-020-0170-0.

202. Yu, C.; Lanjuan, L. SARS-CoV-2: virus dynamics and host response.

The Lancet. Infectious Diseases. Vol. 20, issue 5, pgs. 515-516. May 1, 2020.

203. Shu-ren, L.; Zi-jian, T.; Zai-han, L.; Xuan L. Searching therapeutic strategy of new coronavirus pneumonia from angiotensin-converting enzyme 2: the target of COVID-19 and SARS-CoV.

Eur J Clin Microbiol Infect Dis.2020 Apr 13 : 1-6.doi: 10.1007/s10096-020-03883-y.

204. Andersen, K.G.; Rambaut, A.; Lipkin, W.I.; et al. The proximal origin of SARS-CoV-2. Nat Med 26, 450-452 (2020). https://doi.org/10.1038/s41591-020-0820-9.

205. University of California - Berkeley. "Coronavirus outbreak raises question: Why are bat viruses so deadly? Bats' fierce immune systems drive viruses to higher virulence, making them deadlier in humans.".

ScienceDaily. ScienceDaily, 10 February 2020. <www.sciencedaily.com/releases/2020/02/200210144854.htm>.

206. Ye Q.; Wang B.; Mao J. The pathogenesis and treatment of the 'Cytokine Storm' in COVID-19 [published online ahead of print, 2020 Apr 10]. J Infect. 2020;S0163-4453(20)30165-1. doi:10.1016/j.jinf.2020.03.037.

207. Heneghan, C.; Aronson, J.; Mahtani, R.H.K. Rapidly managing pneumonia in older people during a pandemic. On behalf of the Oxford COVID-19 'Evidence Service Team. Centre for Evidence-Based Medicine, Nuffield Department of Primary Care Health Sciences, University of Oxford.

208. Vigant, F.; Santos, N.C.; Benhur L. Broad-spectrum antivirals against viral fusion. Nat Rev Microbiol. 2015; 13(7): 426-437.doi: 10.1038/nrmicro3475.

209. Mustafaa, S.; Balkhyb, H.; Gabere, M.N. Current treatment options and the role of peptides as potential therapeutic components for Middle East Respiratory Syndrome (MERS): A review. Journal of Infection and Public Health.Volume 11, Issue 1, January-February 2018, Pages 917.https://doi.org/10.1016/j.jiph.2017.08.009.

210. Schoeman, D.; Fielding, B.C. Coronavirus envelope protein: current knowledge. Coronavirus envelope protein: current knowledge. Virol J 16, 69 (2019). https://doi.org/10.1186/s12985-019-1182-0.

211. Khan, R. J.; et al. Targeting Novel Coronavirus 2019: A Systematic Drug Repurposing Approach to Identify Promising Inhibitors Against 3C-like Proteinase and2'-O-Ribose Methyltransferase. ChemRxiv. Preprint. https://doi.org/10.26434/chemrxiv.11888730.v1. 2020.

212. Qiang W.; Ying Z.; Xiaojia C.; An H. Virtual screening of approved clinic drugs with main protease (3CLpro). Preprints (www.preprints.org). Not peer reviewed. March, 8, 2020. .

213. Rabaan A.A.; Al-Ahmed S.H.; Haque S.; et al. SARS-CoV-2, SARS-CoV, and MERS-COV: A comparative overview. Infez Med. 2020;28(2):174-184. 
214. Vicenzi, E.; Canducci, F.; Pinna, D. Coronaviridae and SARS-associated Coronavirus Strain HSR1. Emerg Infect Dis. 2004 Mar; 10(3): 413-418.doi: 10.3201/eid1003.030683.

215. BMJ. Covid-19: trials of four potential treatments to generate "robust data" of what works. $B M J$ 2020;368:m1206. doi: https://doi.org/10.1136/bmj.m1206.

216. Mycroft-West, C.; Dunhao, S.; Stefano E.; et al. SARS-CoV-2 surface S1 Receptor Binding Domain binds heparin. bioRxiv preprint doi: https://doi.org/10.1101/2020.02.29.971093. . 2020.

217. Capila, I., Linhardt, R.J. Heparin-Protein Interactions.

Angew. Chem. Int. Ed. 2002, 41, 390 - 412. http://wwwheparin.rpi.edu/main/app/webroot/files/papers/4f1623c54d6ab5.21304086.pdf.

218. Prabakaran P.; Xiao X.; Dimitrov D.S. A model of the ACE2 structure and function as a SARS-CoV receptor. Biochem Biophys Res Commun. 2004;314(1):235-241. doi:10.1016/j.bbrc.2003.12.081.

219. Yi, C.E.; Ba, L.; Zhang, L.; Ho, D.D.; Chen, Z. Single Amino Acid Substitutions in the Severe Acute Respiratory Syndrome Coronavirus Spike Glycoprotein Determine Viral Entry and Immunogenicity of a Major Neutralizing Domain. Journal of Virology Aug 2005, 79 (18) 11638-11646; DOI: 10.1128/JVI.79.18.11638-11646.2005.

220. Hui, L.; Liang, L.; Dingyu, Z.; et al. SARS-CoV-2 and viral sepsis: observations and hypotheses. The Lancet. Published:April 17, 2020DOI:https://doi.org/10.1016/S0140-6736(20)30920-X.

221. Venkataraman, T.; Frieman, M.B. The role of epidermal growth factor receptor (EGFR) signaling in SARS coronavirus-induced pulmonary fibrosis. Antiviral Res. 2017 Jul; 143: 142-150.doi: 10.1016/j.antiviral.2017.03.022.

222. Wexler, M. PFF Stresses Differences Between COVID-19 and ILD Fibrosis Patterns. Pulmonary Fibrosis News. April 8, 2020. https://pulmonaryfibrosisnews.com/2020/04/08/pff-statement-differencesfibrosis-patterns-between-covid-19-and-ilds/.

223. Bilgin G.; Kismet K.; Kuru S.; et al. Ultrastructural investigation of the protective effects of propolis on bleomycin induced pulmonary fibrosis. Biotech Histochem. 2016;91(3):195-203. doi:10.3109/10520295.2015.1123294.

224. Pesheva, E. Coronavirus and the heart. The Harvard Gazette. April 14, 2020. https://news.harvard.edu/gazette/story/2020/04/covid-19s-consequences-for-the-heart/.

225. Iwasaki, A. and Grubaugh, N.D. Why does Japan have so few cases of COVID-19? EMBO Mol Med (2020)12:e12481. https://doi.org/10.15252/emmm.202012481.

226. Linlin Z.; Daizong L.; Xinyuanyuan S.; et al. Crystal structure of SARS-CoV-2 main protease provides a basis for design of improved $\alpha$-ketoamide inhibitors. Science 24 Apr 2020:Vol. 368, Issue 6489, pp. 409-412. DOI: $10.1126 /$ science.abb3405.

227. Lentile, G. Coronavirus News Roundup: Inhaler Shortages, Updates on Potential Treatments. MJH Life Sciences. Drug Topics. March 27, 2020. https://www.drugtopics.com/health-system-news/coronavirusnews-roundup-inhaler-shortages-updates-potential-treatments.

228. Moran, B. Can Inhaling Nitric Oxide Treat - Or Prevent - COVID-19? MGH Wants To Find Out. Wbur. April 10, 2020. https://www.wbur.org/commonhealth/2020/04/10/nitric-oxide-gas-mass-generalcoronavirus-tests.

229. OINDP News. OINDP News. OINDP News website. [Online] OINDP. Jan 29, 2020. [Cited on: May 5, 2020.] https://www.oindpnews.com/2020/01/pulmotect-says-that-preclinical-testing-of-its-pul-042-inhaledimmune-stimulant-shows-potential-against-coronavirus-infections/. 
230. Pulmotect, Inc. Clinicaltrials.gov. Clinicaltrials.gov website. [Online] NIH U.S. National Library of Medicine, April 14, 2020. [Cited: May 5, 2020.] https://clinicaltrials.gov/ct2/show/study/NCT04312997.

231. Balfour, H. Collaboration to develop inhaled mRNA-based antibody therapy for COVID-19. Drug Target Review News. April 6, 2020. https://www.drugtargetreview.com/news/59425/collaboration-to-developinhaled-mrna-based-antibody-therapy-for-covid-19/.

232. Amirav, I.; Newhouse, M.T. Transmission of coronavirus by nebulizer: a serious, underappreciated risk. CMAJ March 30, 2020 192 (13) E346; DOI: https://doi.org/10.1503/cmaj.75066.

233. O'Neil, C.A.; Jiayu, L.; Leavey, A.; et al. for the Centers for Disease Control and Prevention Epicenters Program, Characterization of Aerosols Generated During Patient Care Activities. Clinical Infectious Diseases, Volume 65, Issue 8, 15 October 2017, Pages 1342-1348, https://doi.org/10.1093/cid/cix535.

234. Pereira, A.S.; Seixas, F.R.M.; Neto, F.R.A. Propolis: 100 Years of Research and Future Perspectives. Quím. Nova vol.25 no.2 São Paulo Abr./May 2002. https://doi.org/10.1590/S0100-40422002000200021.

235. SEBRAE. SEBRAE. m.sebrae.com.br. [Online] 2017. [Cited on: May 5, 2020.] https://m.sebrae.com.br/Sebrae/Portal\%20Sebrae/UFs/BA/Anexos/Produ\%C3\%A7\%C3\%A3o\%20de\%20pr\%C3\%B3polis\%20

236. Bogdanov, S.; Bankova, V. Propolis: Origin, Production, Compostion. Bee Product, Science. (Research Gate)

https://www. researchgate.net/publication/304012141_Propolis_Origine_Production_ Composition.

237. Beehour. beehour.com. Beehour website. [Online] Beehour. [Cited on: May 05, 2020.] https://beehour.com/how-long-does-it-take-to-get-honey-from-a-new-hive/.

238. Lewis, T. Here's What We Know about the Most Touted Drugs Tested for COVID-19. Scientific America. April 16, 2020. https://www.scientificamerican.com/article/heres-what-we-know-about-the-most-touteddrugs-tested-for-covid-191/.

239. Russell, B.; Moss, C.; Rigg, A.; Hemelrijck, M.V. COVID-19 and treatment with NSAIDs and corticosteroids: should we be limiting their use in the clinical setting? Ecancermedicalscience. 2020; 14: 1023.doi: 10.3332/ecancer.2020.1023.

240. Fan-ching, L.; Young, H.A. Interferons: Success in anti-viral immunotherapy. Cytokine Growth Factor Rev. 2014 Aug; 25(4): 369-376. doi: 10.1016/j.cytogfr.2014.07.015.

241. Dogan, A.; Otlu, S.; Celebi, O.; et. al. An investigation of antibacterial effects of steroids. Turkish Journal of Veterinary and Animal Sciences. Turk J Vet Anim Sci (2017) 41: 302-305. doi:10.3906/vet-1510-24.

242. Kaplan, A.; Lee,M.W.; Wolf, A.J.; et. al. Direct antimicrobial activity of Interferon- $\beta$. J Immunol. 2017 May 15; 198(10): 4036-4045. doi: 10.4049/jimmunol.1601226.

243. Chinappi, M.; Via, A.; Marcatili, P.; Tramontano, A. On the Mechanism of Chloroquine Resistance in Plasmodium falciparum. PLoS One. 2010; 5(11): e14064. Published online 2010 Nov 19. doi: 10.1371/journal.pone.0014064. 